\title{
Microfluidic Device Design for Selective Enrichment of Biocolloids Based on their Deformability and Polarization
}

\author{
A Dissertation \\ Presented to \\ the faculty of the School of Engineering and Applied Science \\ University of Virginia
}

\author{
in partial fulfillment \\ of the requirements for the degree \\ Doctor of Philosophy
}

by

Walter Brendan Varhue

May 2017 


\title{
APPROVAL SHEET
}

\author{
This Dissertation \\ is submitted in partial fulfillment of the requirements \\ for the degree of \\ Doctor of Philosophy
}

Author Signature:

This Dissertation has been read and approved by the examining committee:

Advisor: Prof. Nathan Swami

Committee Member: Prof. Lloyd Harriott

Committee Member: Prof. Shayn Peirce-Cottler

Committee Member: Prof. Rebecca Pompano

Committee Member: Prof. Edward H. Bertram

Committee Member:

Accepted for the School of Engineering and Applied Science:

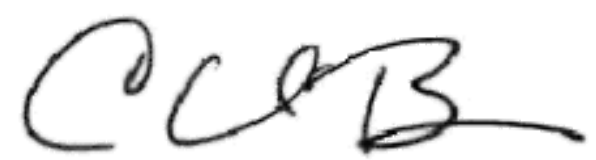

Craig H. Benson, School of Engineering and Applied Science

May 2017 


\section{Abstract}

The development of methods to achieve the selective enrichment and separation of biocolloids based on particle size, shape, biomechanical properties, cell phenotype, and biomolecular conformation is of great interest towards application in tissue regeneration and bioanalytical systems. Microfluidic systems enable the selective translation of particles by hydrodynamic means based on size, shape and deformability. Furthermore, separation can be achieved through the integration of electrokinetic methods such as dielectrophoresis (DEP), which enables frequency selective translation of bioparticles based on dielectric parameters, including intracellular electrophysiology, surface topology, and bimolecular conformation. Herein we develop microfluidic device platforms for two clinically significant applications: (1) deformability-based separation of pancreatic islets of Langerhans from exocrine acinar tissue; and (2) simultaneous enrichment and detection of proteomic biomarkers in physiological media.

Diabetes remains the seventh leading cause of death among Americans. A potential cure for the type-1 variant, which is currently managed through lifelong exogenous insulin administration, can be achieved through transplantation of pancreatic islet of Langerhans to restore normal endocrinal function. However, endocrine islet tissue forms a small proportion of the pancreas ( 1\%), and must be isolated from the contaminating exocrine acinar tissue that makes up the majority of the organ. Herein, we demonstrate that the density gradient based method that is currently used to separate islets from acinar tissue causes the islets to be sparsely distributed across a wide array of centrifuged sample bins of varying purity. The resulting transplant plug contains significantly high levels of contaminating exocrine acinar tissue ( $40 \%$ of transplant volume), thereby exacerbating immune responses and requiring the life-long administration of immune suppressants after transplantation. In comparison to the significant size and density overlaps between the islet and acinar tissue populations, we demonstrate that their deformability overlaps are minimal. Utilizing this feature, we demonstrate a microfluidic separation strategy, wherein tangential flows enable selective deformation of acinar populations towards the waste stream and sequential switching of hydrodynamic resistance enables the collection of rigid islet cell aggregates. This system is shown to be capable of enriching islets from relatively dilute starting levels up to purity levels that meet transplant criteria, as well as towards enhancing islet purity from the starting samples obtained after density gradient based separation.

The electrokinetic trapping of nanoscale biocolloids and biomolecules within nanoslit devices enables high degrees of enrichment and overcomes mass transport limitations within various sensing modalities. Such nanoslit device architectures are applied to enable the simultaneous trapping and detection of two important proteomic biomarkers: (i) Neuropeptide $\mathrm{Y}$ (NPY), which offers noninvasive diagnostic information on stress, depression and neurotrauma; and (ii) prostate specific antigen, an important biomarker for diagnosis prostate cancer. Herein, we demonstrate the fabrication and assembly of nanoslit devices for the electrokinetic enrichment of these biomarkers through frequency selective dielectrophoresis (DEP), which is integrated with a graphene modified surface for electrochemical detection post-enrichment. 


\section{Acknowledgments}

First I would like to express my sincerest gratitude to my advisor Dr. Nathan Swami. Thank you for taking a chance on me as, first a masters, and then PHD student. Thank you for your advice and guidance, not only on how to advance my research, but also on how best to organized and present my results. Thank you for your patience and guidance throughout these many years. This dissertation would not have been possible without you.

Thank you also to my many group mates and colleagues for your valuable advice discussion, help, and friendship. To Ali Rohani, Dr. Rachel Su, Dr. KT Liao, Armita Salahi, John Moore, Dr. Bankim Sanghavi, Vahid Farmehini, Karina Torres, and Dr. Frank Block thank you. Special thanks; to Dr. KT Liao for your early mentorship and guidance in microfabrication and microfluidics; to Dr. Bankim Sanghavi for your guidance and help with the Nanoslit project; and to Dr. Frank Block for your help in finding my feet in Charlottesville. Extra special thanks to Ali Rohani and Dr. Rachel Su who began this journey together with me and without whom I would not have made it so far. Also special thanks to everyone for putting up with Ali and I arguing constantly during group meetings.

I would also like to thank my collaborators on the Islet project. To Prof. Shayn Peirce-Cottler and Dr. Kenneth Brayman thank you for your support and advice. To Molly Kelly Goss and Morgan Lataillade thank you for your brilliant work with the mouse angiogenesis model. Special Thanks to Linda Langman for your invaluable help and guidance with both the human and mouse islets, without your help none of this would have been possible.

I would also like to thank Prof. Chia Fu Chou and his group for their hospitality during my stay in Aademia Sinica and your guidance on the Nanoslit project. Specia thanks to Prof. Leo Lesser-Rojas for your help with nanoslit fabrication and operation and to Dr. Yii-Lih Lin for guiding me through the cleanroom and the city.

I would also like to thank to my friends who supported me through my time here and before. To Jon Paquette, Andrew Lutton, and Andy French thanks for always having my back. To Korey King, Blanton Bryant, Meghan Prendergast, Dustin Harris, and Aaron Barclay thanks for keeping me sane.

Finally, to my family; My mother Mary Ellen, my father Walter, my brother Tim, my sister Nora and my wife Kazusa. There is not adequate space in the universe to express what needs to be said so I will simply say thank you. 


\section{Dedication}

For My father

Who has always been my inspiration and role model 


\section{Table of Contents}

Abstract $\quad$ i

Acknowledgments ii

Dedication iii

Publications vii

$\begin{array}{ll}\text { Chapter 1: Background } & 1\end{array}$

$\begin{array}{lr}\text { Introduction } & 1\end{array}$

Aim One Background: Deformability based separation of islets 4

$\begin{array}{ll}\text { Type } 1 \text { Diabetes } & 4\end{array}$

Transplantation of Pancreatic Islets of Langerhans 5

$\begin{array}{ll}\text { Microfluidic methods of separation } & 7\end{array}$

$\begin{array}{lr}\text { Hydraulic Linear Circuit Analogy } & 9\end{array}$

$\begin{array}{ll}\text { The measurement of deformability for cellular aggregates } & 10\end{array}$

$\begin{array}{ll}\text { Hydraulic circuit model for the } P_{\text {th }} \text { measurement device } & 11\end{array}$

Size and deformability based separation of cellular aggregates $\quad 14$

$\begin{array}{ll}\text { Design criteria for Separation Device } & 16\end{array}$

$\begin{array}{ll}\text { Hydraulic circuit model optimization of channel dimensions } & 17\end{array}$

$\begin{array}{ll}\text { Need for focusing flows in separation device } & 19\end{array}$

Aim Two Background: Electrokinetic preconcentration and 22

electrochemical detection within a nanoslit platform

$\begin{array}{ll}\text { Dielectrophoresis } & 22\end{array}$

Negative Dielectrophoretic (nDEP) Dam Method 24

$\begin{array}{ll}\text { Chapter 2: Methods } & 26\end{array}$

Aim One Methods: Deformability based separation of islets 26 
Human pancreas digestion and density gradient

purification

Mouse Islet isolation

Viability and insulin production assays

Experimental setup: Microfluidic separation of islets

High aspect ratio Microdevice fabrication

Measurements of islet viability and vascularization

Aim Two Methods: Electrokinetic preconcentration and electrochemical detection within a nanoslit platform

Fabrication of fused silica Nano constrictions within a Nano-slit

Integration of electrochemical detection electrodes

Nanofluidic device operation for ultra-fast NPY detection

Nanofluidic device operation for ultra-fast PSA detection

Modification of secondary PSA antibody for sandwich assay

Aim One Results: Deformability based separation of islets

Size and deformability overlaps for mouse islets versus acinar particles

Microfluidic separation of islet versus acinar particles

Viability of islets isolated by microfluidic device

Size and deformability overlap human derived islets

versus acinar particles

Aim Two Results: Electrokinetic preconcentration and electrochemical detection within a nanoslit platform 
Preconcentration and electrochemical detection of PSA

within a nano-slit array

Influence of PSA enrichment on binding kinetics to anti-PSA

54

Enhancing sensitivity for PSA detection

Chapter 4: Conclusion and Future Work

Conclusion

58

Aim One Future Work: Deformability based separation of islets

60

Adaptation of separation device to human Islet

60 and acinar samples

Aim Two Future Work: Electrokinetic preconcentration and electrochemical detection within a nanoslit platform

Drawbacks of current fabrication methods and materials 


\section{List of Publications}

[1] Y. H. Su, M. Tsegaye, W. Varhue, K. T. Liao, L. S. Abebe, J. A. Smith, et al., "Quantitative dielectrophoretic tracking for characterization and separation of persistent subpopulations of Cryptosporidium parvum," Analyst, vol. 139, pp. 66-73, 2014.

[2] A. Rohani, W. Varhue, Y. H. Su, and N. S. Swami, "Quantifying spatio-temporal dynamics of biomarker pre-concentration and depletion in microfluidic systems by intensity threshold analysis," Biomicrofluidics, vol. 8, Sep 2014.

[3] B. J. Sanghavi, W. Varhue, J. L. Chavez, C. F. Chou, and N. S. Swami, "Electrokinetic Preconcentration and Detection of Neuropeptides at Patterned Graphene-Modified Electrodes in a Nanochannel," Analytical Chemistry, vol. 86, pp. 4120-4125, May 62014.

[4] A. Rohani, W. Varhue, Y. H. Su, and N. S. Swami, "Electrical tweezer for highly parallelized electrorotation measurements over a wide frequency bandwidth," Electrophoresis, vol. 35, pp. 1795-1802, Jul 2014.

[5] B. J. Sanghavi, W. Varhue, A. Rohani, K. T. Liao, L. A. L. Bazydlo, C. F. Chou, et al., "Ultrafast immunoassays by coupling dielectrophoretic biomarker enrichment in nanoslit channel with electrochemical detection on graphene (vol 15, pg 4563, 2015)," Lab on a Chip, vol. 15, pp. 4626-4626, 2015.

[6] A. Rohani, W. Varhue, K. T. Liao, C. F. Chou, and N. S. Swami, "Nanoslit design for ion conductivity gradient enhanced dielectrophoresis for ultrafast biomarker enrichment in physiological media," Biomicrofluidics, vol. 10, May 2016.

[7] W. Varhue, L. Langman, D. Bowers, S. Peirce-Cottler, N. Swami, and K. Brayman, "A Novel Microfluidic Device for Purification of Pancreatic Islet Preparations.," American Journal of Transplantation, vol. 16, pp. 715-715, Jun 2016. 


\section{Chapter 1: Background}

\section{introduction}

Microfluidics is the study and design of devices to manipulate the flow of minute volumes of liquid, usually in the nano to picolitre range, through geometries wherein at least one dimension is limited to under a millimeter in scale. Growing out of the MEMS field after the development of micro flow sensors, pumps, and switches in the late 1980's, the field of microfluidics has seen rapid development and growth over the last three decades. Though seemingly simple in concept the true utility of these platforms arise from the low Reynold's numbers associated with micron-scale flows and environments. At low Reynold's numbers, viscosity dominates over inertia to result in laminar flow, wherein predictable parallel flow streams enable high precision and selective translation of suspended particles based on characteristics such as size, shape and deformability. Further analyte manipulation is possible through the integration of external fields, which are used to selectively translate target particles using mechanisms such as AC or DC electrokinetics, acoustophoresis, and magnetophoresis[5].

Due to this utility, microfluidic platforms have found applications in a range of diverse fields such as tissue regeneration, optics, and analytical chemistry. For biomedical applications, such as cell based therapies and bioanalytical systems, such methods may be leveraged to achieve the selective enrichment and separation of biocolloids based on particle size, shape, biomechanical properties such as deformability, cell phenotype, and bimolecular conformation in a high precision and label free manner[57]. Herein, we demonstrate the development of microfluidic device platforms for two clinically significant applications. The first is the deformability-based separation of pancreatic islets of Langerhans, a specialized cell aggregate used in transplant therapies for type one diabetes, from a common contaminant, exocrine acinar tissue. The second is the enrichment and detection of two proteomic biomarkers that are present at trace levels in physiological media ( pg/mL): (i) Neuropeptide Y (NPY), which offers noninvasive diagnostic information on stress, depression and neurotrauma; and (ii) prostate specific antigen, an important biomarker for diagnosis prostate cancer. 
Diabetes remains the seventh leading cause of death among Americans and is rapidly on rise within several emerging economies around the world. A potential cure for the type-1 diabetes (T1D) variant, which is currently managed through lifelong exogenous insulin administration, is through the transplantation of pancreatic islet of Langerhans. A component of these specialized cell aggregates are the insulin secreting beta cells, which are destroyed by autoimmune response in T1D subjects. Beta cell replacement through transplantation can restore normal endocrinal function. However, endocrine islet tissue forms a small proportion of the pancreas $(\sim 1 \%)$, and must be isolated from the contaminating exocrine acinar tissue that makes up the majority of the organ. The current method using density-based isolation is inadequate, since it produces transplant plugs of relatively low purity and suffers from substantial sample loss due to imperfect separation. Herein, we demonstrate a microfluidic separation strategy utilizing deformability differences in the harvested islet tissue and the contaminating acinar tissue. We envision the application of this microfluidic strategy as a secondary separation step recovering lost islet material from low purity waste samples, as well as for generating small-volume transplant plugs, with high islet purity and significantly reduced acinar levels for the purpose of alleviating immune responses after transplantation.

Prior deformability based separation methods for single cells involved particles with a tight size distribution and of similar shape. The analogous operation of separating multi-cell aggregates, such as islets and acinar tissue, is challenging due to the far broader range of size and shape distributions of cellular aggregates. Herein, we address this challenge by dynamic balancing of flow resistances to account for the wide size and shape distributions of multi-cell aggregates. Specifically, the minimal overlap in deformability levels of islet versus acinar tissue aggregates is used to enable selective isolation of islets, by setting the pressure differential over a series of microfluidic bifurcating junctions to be just sufficient for deforming the acinar tissue through to the waste port, while the dynamic adjustment of flow resistance enables the collection of the rigid islets under tangential flow. In this manner, we demonstrate that this microfluidic method for islet isolation can potentially address the shortcomings of the density gradient isolation method to generate transplant samples with higher islet purity. The viability of the islets 
isolated by the microfluidic method is validated by propidium iodide staining and their ability for angiogenesis is verified using a murine retinal model[8, 9],[10].

In the arena of biomolecular sensing, limitations imposed by mass transport of the analyte molecules towards the sensing region are generally accepted to be a major factor limiting the performance, sensitivity, and speed under numerous sensing modalities. This is especially the case for miniaturized sensor platforms and for situations requiring the detection of subnanomolar concentration levels of analyte [4, 11-14]. To alleviate this significant bottleneck, there is a need to develop methods to enhance analyte flux towards the surface of the sensor array that is usually immobilized with receptors. $[3,15]$ This enhancement may be achieved through the integration of a method to actively drive new analyte material to the sensing region, thereby achieving sample enrichment and replenishment. Further enhancement may be achieved through the use of a nano-slit device geometry. Diffusion lengths within a nanochannel or nanoslit (a channel with at least one dimension in the nanoscale) are constrained by the nanoscale confinement. For this reason, sensor arrays within a nanoslit benefit from high radial diffusion of analyte to the sensor surface, resulting in faster response time and improved sensitivity.

Herein, we developed a nanoslit platform for the integration of AC electrokinetic preconcentration using dielectrophoretic molecular damming with electrochemical detection of the enriched analyte. [2-4] This preconcentration method utilizes a balance between electrokinetic forces to localize sample particles within extremely small channel volumes and has demonstrated the potential to achieve a million-fold degree of concentration enrichment within a few seconds. Through the combination of these two enhancement methods, improvement of assay times and sensitivity is confirmed by using electrochemical sensing modalities. 


\section{Aim One Background: Deformability based separation of islets}

\section{Type 1 Diabetes:}

Diabetes mellitus (DM) is a group of metabolic diseases in which blood glucose levels are held at an elevated level over a significant period of time, thereby causing a range of glycol-metabolic complications. The Type 1 variant (T1D) represents the most severe form of diabetes, and is caused by an autoimmune response that selectively targets the insulin producing $\beta$-cell components of pancreatic islet of Langerhans (pancreatic islets), which are a specialized set of cell aggregates in the pancreas. The resulting progressive $\beta$-cell destruction leads to the degradation and eventual cessation of insulin production and secretion, a process that is vital to the regulation of blood glucose levels. This failure of the endocrine function of the pancreas eventually results in glycol-metabolic dysregulation, thereby causing a range of complications associated with long-term elevation of blood glucose, including diabetic retinopathy, neuropathy, nephropathy, and cerebrovascular and cardiovascular disease.[16]

T1D remains a major cause of morbidity and mortality worldwide. In 2012 it cost an estimated $\$ 245$ billion dollars to treat and manage diabetes in the US alone. This constitutes a $40 \%$ increase in cost related to the disease from just five years earlier with an estimated $\$ 174$ billion spent in 2007. [17] For a person suffering from DM the average healthcare expenditures surmount $\$ 13,700$ annually, roughly 2.3 times higher than expenditures for individuals without diabetes. While Type 2 Diabetes (T2D) is caused by a chronic fuel surfeit and accounts for nearly $90 \%$ of DM cases, the costs associated with T1D treatment and management are consistently higher on a per case basis.[18] Current long term management of T1D is achieved through lifelong insulin administration in order to synthetically regulate blood glucose and achieve energy homeostasis. However, while this therapy may generally prevent or even reverse long term T1D complications in some patients, it fails to replicate the sensitivity of glucose regulation achieved by a healthy pancreatic endocrine function, thereby leaving subjects vulnerable to severe episodes of hypoglycemia and hypoglycemic unawareness. As such, there is a need to develop new long term treatment methods that focus on the restoration of insulin production and blood glucose management for effectively alleviating T1D symptoms. 


\section{Transplantation of Pancreatic Islets of Langerhans}

One potential treatment option which may achieve this goal is the transplantation of tissues from a healthy set of pancreas. In spite of the promising nature of this treatment, it is associated with several major drawbacks, which limit its viability as a general cure. These drawbacks include the need for longterm immunosuppression therapy, as well as the heightened risk of mortality and morbidity associated with major transplant surgery. [19] Due to these risks, pancreas transplantation is often limited to a select group of individuals who have either already undergone successful kidney transplantation or are receiving simultaneous kidney-pancreas transplants and therefore already require immunosuppressant drugs. Furthermore, due to the risks involved, and the rarity of transplantable organs, high risk individuals, including elderly patients or those suffering from cardiovascular disease, are usually excluded during the patient screening process.

Whole organ transplantation is limited in its application as a long-term cure for T1D due to the numerous risks associated with major surgery, as well as the general scarcity of quality transplantable organs. First proposed in 1967, $\beta$-cell replacement through the transplantation of pancreatic islets of Langerhans has emerged as a potentially general method to return insulin independence to a T1D subject. Pancreatic islets are specialized cell aggregates containing $\beta$-cells vital to the production and regulation of insulin. Transplantation of these pancreatic islets is often far less invasive than full organ transplantation, with the Collaborative Islet Transplant Registry (CITR) recently reporting a 20-fold lower morbidity risk from the peri-procedural complications associated with transplantation of islets as compared to those associated with whole organ transplantation. Such transplantations have been shown to result in restoration of sensitive insulin secretion and associated glucose disposal. It offers a range of benefits to recipients including a decrease in insulin dose requirements, improved glycated hemoglobin levels, and an overall reduction in incidences of hypoglycemic episodes.[16, 20] Furthermore this method may utilize pancreatic islets derived from donor organs considered to be of too low a quality for whole organ transplantation, which is a significant benefit due to scarcity of transplant material. Due to the potential improvements over whole organ transplantation in the areas of patient ease, safety, procedural outcome, 
and efficiency in use of scarce donor material, pancreatic islet transplantation has the potential to become a leading cure for T1D.[16] However, the long term efficacy of islet transplantation therapies are limited by their high cost, poor sample purity and exacerbated immune responses associated with the transplantation, which requires the life-long administration of immune suppressants, preventing this potentially curative procedure from seeing wider implementation.

Currently the insulin independence in recipients of pancreatic islet transplants is only maintained for short time periods post transplantation. This effective time frame appears independent of the number of islet transfusions given to a patient and only very rarely extends beyond five years after the initial transplant.[21] This transplant failure is largely attributed to the inconsistent transplant purity and quality, leading to a heightened immune response, poor engraftment of the transplant material and eventual rejection by the subject. This lack of long term efficacy is compounded by the costs associated with the transplant procedure. The current transplant preparation method suffers from yield issues. A healthy pancreas is estimated to contain around 2 million islets. Current isolation techniques typically result in yield of 800,000 islets a loss of over $50 \%$ of transplantable material. This low yield necessitates the use of multiple donor organs to produce enough islet tissue volume to perform a single transplant. This requirement not only greatly increases the cost of an individual procedure, but further exacerbates immune response as tissue from multiple downers is present in the final transplant.

A primary cause of the exacerbated immune response is the low purity of islets within the packed tissue volume that is transplanted. The pancreas is largely comprised of two tissue types: hormone producing islets that are a specialized aggregate of embedded endocrine cells (comprising just 1-2 percent of the pancreas) and surrounding exocrine tissue that produces digestive enzymes. In the current isolation protocol for the transplant sample from donor pancreas[22], the pancreatic tissue is digested in a ricorti chamber by an enzymatic digestion to generate a mixture of islets and acinar tissue, which are then separated by a continuous density gradient (DG) purification step to generate the transplant sample. However, the insubstantial density difference between islets versus the acinar tissue, and the size heterogeneity of the islets, causes the islets to be spread over a succession of the collected density 
gradient fractions that widely vary in purity levels. As a result, in order to obtain sufficient numbers of islets in the transplant sample, fractions with various ranges of density and purity must be combined, thereby causing the transplant sample to have a high packed tissue volume with poor levels of islet purity. Furthermore, sample fractions containing above $30 \%$ acinar tissue are unusable for transplantation. This typically results in a loss of $15 \%$ of available islet material retained after the digestion step. Before pancreatic islet transplantation can achieve its potential as a cost effective cure for T1D these issues of transplant quality and isolation efficiency must be resolved.

To address these issues of sample loss and generate transplant plugs with substantially higher islet purity, we demonstrate a method for microfluidic separation of islets from acinar tissue, wherein differences in the deformability of the respective cellular aggregate play a significant role. Since islets contain large levels of the skeletal extracellular matrix (ECM) structure versus acinar tissues that possess minimal ECM[23], islets are less damaged by the enzyme used within the digestion step, thereby causing islets to be more rigid than acinar tissue following the digestion step. We show that the deformability overlaps between islet and acinar tissue following enzymatic digestion are minimal in comparison to their substantial size and density overlaps. As a result, their separation is accomplished by utilizing tangential flows at an array of microfluidic bifurcations that are designed to deform the acinar tissue towards a waste port and collect the rigid islet populations to form a high purity transplant plug.

\section{Microfluidic methods of separation}

Microfluidic methods have been widely applied towards separation of individual cells based on their size[24], shape[25] and deformability[26] characteristics, such as blood cells from healthy versus from leukemia patients[27], oral squamous cells from healthy versus cancerous patients[28] and to distinguish the lineage of stem cells at various differentiation stages[29]..[30, 31] These methods widely rely on the low Reynolds (Re) and Stokes (St) numbers associated with microfluidic systems.[30] Reynolds numbers are a non-dimensional factor that describes the ratio of inertial and viscus forces in a flow as given by the following equation:

$$
R e=\frac{U D_{h}}{v}
$$




\section{Equation 1}

Where $U$ is the average fluid velocity, $D$ is the characteristic dimension of a channel or obstacle, and $v$ is the kinetic viscosity $\left(10^{-6} \mathrm{~m} / \mathrm{s}^{2}\right.$ for water). A flow is said to be laminar in cases wherein the Re of a flow drops below a threshold value ( $\left(\mathrm{R}_{\text {trans }}\right)$ where, for a given circumstance, viscosity dominates over inertia. Typical $\mathrm{Re}_{\text {trans }}$ values range from 1,000 to 2,000 for fluid flowing through a channel and 65 to 100 for fluid flowing around an object. The Stokes number is a dimensionless factor describing how quickly a particle will react to changes in the carrier stream. This factor is given by the following equation.

$$
S t=\frac{\rho_{p} a^{2} / 18 \mu}{D_{h} / U}
$$

\section{Equation 2}

Here, $a$ is the diameter of the particle, $\rho_{p}$ is the densitiy of the particle, and $\mu$ is the viscosity of the carrier fluid. In cases of low Stokes numbers, the particle will react rapidly to changes in its carrier stream. In the case of flows through microfluidic systems, Reynolds numbers are typically extremely low due to the small geometric dimensions of the channels, usually ranging between 1 and $10^{-4}$. [32] Similarly, in the case of microparticles such as cells or other biocolloids, their Stokes numbers are also typically low. In cases where the Re of the carrier fluid flow and the St of the particle is small, the trajectory of a particle will follow the streamline of the flow passing through its center of gravity.

Utilizing these phenomena three broad mechanisms of separation are available. These are separations utilizing particle to wall interactions, particle to flow interactions and particle to field interactions. Methods utilizing the particle to wall mechanism rely on interaction with rigid structures within the microfluidic device. These interactions may be used to exert a deterministic force on a particle, thereby achieving particle distinction or separation based on parameters such as particle size or deformability. Examples of such devices are those utilizing micro-post bump arrays[33], micropost filters[34, 35], or pinched flow fractionation[24]. Methods utilizing particle to flow interactions rely on hydrodynamic forces to achieve separation. These methods include hydrodynamic spreading[24], spiraling or curved channel designed to take advantage of dean flow[36], hydrodynamic filtering[37], and 
bifurcation methods[38]. The final mechanism relies on the use of an external force field to achieve separation. This method is most commonly used in Field Flow Fractionation device which have utilized gravity, magnetic, and electrokinetic force fields to achieve separation[39].

\section{Hydraulic Linear Circuit Analogy}

The use of an electrical circuit analogy to model fluid flow through microfluidic devices has been well demonstrated and has proved a valuable tool for the design of microfluidic devices [ref]. As is outlined by Hagen-Poiseuille's law, the volumetric flow rate of an incompressible liquid in laminar flow through a microfluidic channel is linearly proportional to the pressure differential over the channel length. This is analogous to the behavior of electrical current through a resistor as outlined by ohms law,

$$
V=I R ; \Delta p=Q R_{h}
$$

\section{Equation 3}

Here, the volumetric flow rate $(Q)$ corresponds to current $(I)$, the pressure differential $(\Delta p)$ corresponds to voltage $(V)$, and the hydrodynamic resistance $\left(R_{h}\right)$ corresponds to the electrical resistance $(R)$. [40]Utilizing this analogy, standard electrical circuit analysis methods may be used to model fluid flow through a microfluidic device. In order to utilize this analogy, the various components of a microfluidic device must be represented as standard circuit elements, with pumping sources represented as either voltage (constant pressure sources such as pneumatic or vacuum sources) or current (constant flow rate sources such as syringe pumps) sources, whereas tubing, reservoirs, microfluidic channels and other structures are represented as resistor elements. For a straight length of tubing or channel with a constant circular cross section, the hydrodynamic resistance is described as follows:

$$
R h=\frac{\Delta p \pi r^{4}}{8 \mu l}
$$

\section{Equation 4}

Where $r$ is the radius of the circular cross section, $\mu$ is the viscosity of the liquid, and $l$ is the length of the tubing.[40] However, due to the planar nature of standard fabrication methods the cross sections of most 
microfluidic structures are not circular, with most having either a square or rectangular cross section. The hydraulic resistance of a rectangular channel may be approximated as follows

$$
R_{h}=\frac{12 \mu l}{h^{3} w\left(1-.63 \frac{h}{w}\right)}
$$

\section{Equation 5}

In cases when $h<w$, where $h$ is the channel height and $w$ is the channel width. [40] For a structures where the width of the channel varies along the length such as at constriction the hydrodynamic resistance may be given as

$$
R_{h}=\frac{12 \eta}{h^{3}} \int_{0}^{l} \frac{1}{w(x)\left(1-.63 \frac{h}{w(x)}\right)}
$$

\section{Equation 6}

In cases when $h<w$ and

$$
R_{h}=\frac{12 \eta}{h} \int_{0}^{l} \frac{1}{w(x)^{3}\left(1-.63 \frac{w(x)}{h}\right)}
$$

\section{Equation 7}

In cases wherein $h>w$, with $w(x)$ represented as a function that describes the channel width as a function of length. Herein the haudralic circuit analogy is used to model the fluid flow through our microfluidic devices. It allows us to optimize design parameters such as channel, the volumetric flow rate applied at device inlets, outlet resistances in a fast intuitive way. More specifically this method was used to both relate and optimize the applied pressure differential of the device used to measure tissue deformability to a flowrate through our separation device to achieve high throughput separation of islets from acinar tissue.

The measurement of deformability for cellular aggregates 


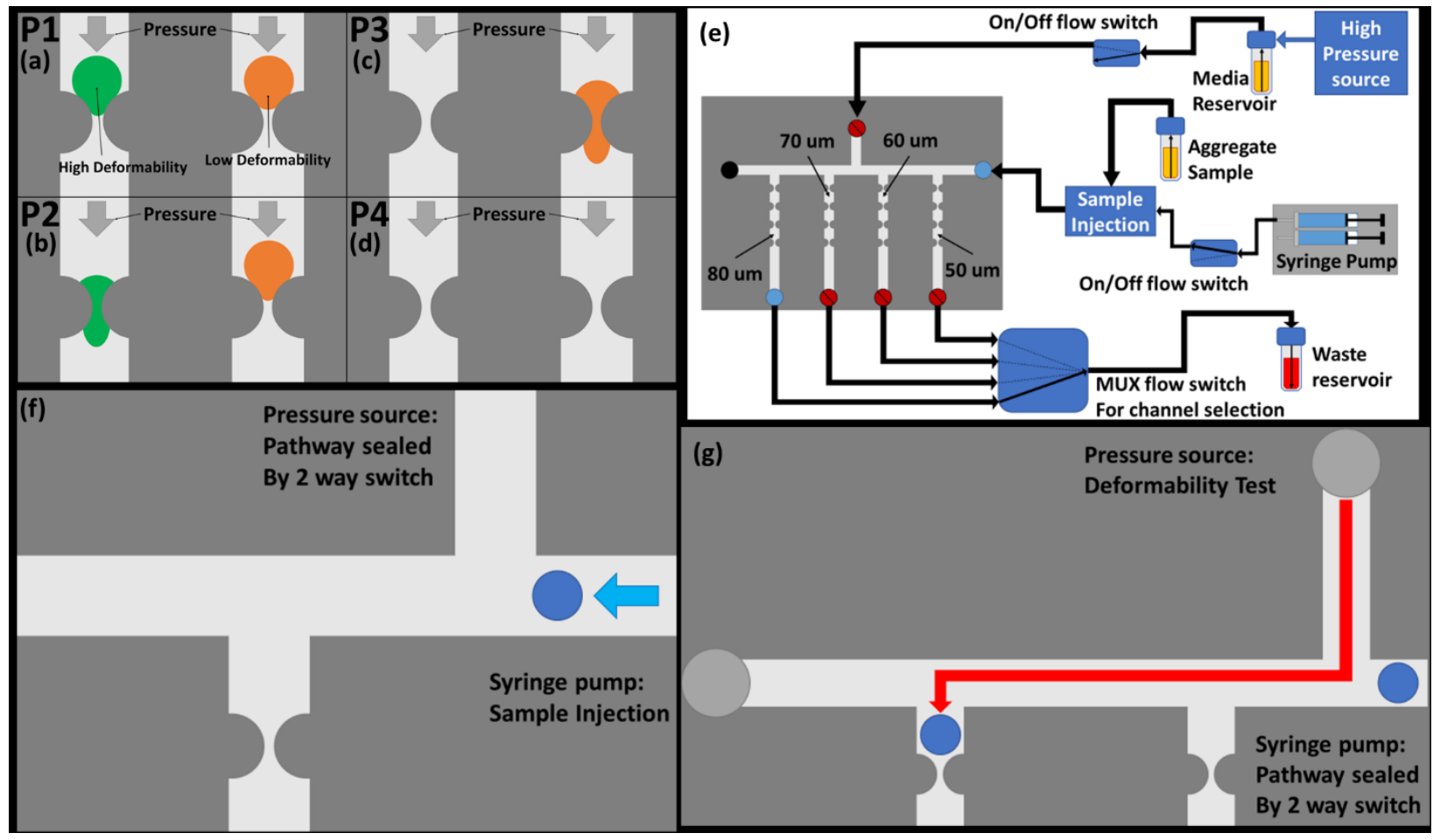

Figure 1: Threshold bypass pressure measurements: (a-d) under successively increasing pressure from P1 to P4, more deformable particles (green) pass through the constriction at lower pressure (P2) than the lower deformability particles (orange); (e) overall device design and fluidic set-up; (f) syringe pump is used to inject sample; (g) individual particles driven under pressure flow towards constriction of interest.

Techniques such as atomic force microscopy[41], optical stretching[42] and micropipette aspiration[43] have long been utilized to measure the deformability of individual cells. While these techniques offer the ability to precisely measure particle elasticity through the application of $\mathrm{pN}$ to $\mu \mathrm{N}$ range force, they are extremely time consuming, low throughput, and require expensive equipment and expertise[44]. Furthermore, recent work has demonstrated that the Young's Modulus of cellular aggregates, such as islets and acinar tissue, is more strongly dependent on intercellular mechanical properties, rather than on the elasticity of the individual components within the cells[45]. Hence, methods to gauge deformation based on the application of force over small areas are likely to be ill-suited to gauge the deformability of cellular aggregates. In recent years, several high throughput microfluidic techniques for measuring deformability differences between individual cells have been developed[44],[46], wherein pressure driven flow across constricting structures is used to induce particle deformation, as measured by particle transit time, threshold bypass pressure[26], induced hydrodynamic or electrical resistance[47], 
and particle shape alterations under shear flow[48]. Since these microfluidic methods probe the ability of the particle to deform through a constriction, they can be more easily translated towards a separation application than other characterization methods. However, multi-cell aggregates, such as islets and acinar tissue are spread over a far broader range of size and shape distributions than individual cells. Hence, metrics such as transit time, induced hydrodynamic resistance and induced electrical resistance are inappropriate means to describe deformability of cellular aggregates; since they require a high enough pressure to reliably deform all of the tested particles, whereas cellular aggregates that possess lower yield strength than individual component cells[45], can disintegrate at the higher pressure levels. This feature also obviates the use of methods based on observing particle shape alterations upon repeated or continuous deformation under shear flow. Hence, we herein choose to measure the threshold bypass pressure, i.e. the minimum pressure required to deform the respective aggregate through a constriction, as the metric for deformability differences between islet and acinar tissue populations. While being of lower throughput than continuous deformation methods, this method is preferred, since it limits the applied pressure to the minimum level required to deform the cell aggregate and the measurement only requires single deformation events. While constriction designs that cause $100 \%$ occlusion for all the tested particles are preferred within prior work on cells[26], since the pressure differential across the particle rises sensitively with deformability differences between particles, this occlusion level cannot be reliably implemented for islets and acinar populations due to their wide size range (100-250 $\mu \mathrm{m})$ and shape distributions. Instead, in order to maintain a relatively similar pressure differential across the tested particles, we choose constriction designs that create up to $75 \%$ occlusion, since the pressure differential across the trapped particle varies linearly within a similar order of magnitude for occlusions up to 75\%[49]. In this occlusion regime, while the bypass pressure will vary with aggregate size and shape, this metric can be translated into the required pressure to achieve micropore separation of islets versus acinar populations, based on their deformability differences, even though they exhibit wide and overlapping size distributions. As per Figure 1a-1d, cellular aggregates are individually translated within a batch flow microfluidic device towards the test constriction that is designed to cause no more than $75 \%$ particle occlusion, so that pressure driven flow can be successively increased to measure threshold bypass 
pressure at which it passes through the constriction. The optimal device is a channel with $400 \mu \mathrm{m}$ width and $350 \mu \mathrm{m}$ height, with a lateral constriction of $80 \mu \mathrm{m}$ that represents $80 \%$ of the smallest islet diameter in the lateral dimension and $150 \%$ of the largest islet diameter in the vertical dimension, thereby ensuring no more than $75 \%$ occlusion in the constriction region.

\section{Hydraulic circuit model for the threshold bypass pressure measurement device}

In order to calibrate the flowrates used in the multichannel continuous flow separation device it is necessary to determine the flowrate through the constriction geometries of the single channel batch mode device for the experimentally measured pressures. In order to do this a hydrodynamic model to the single channel device was created.

The layout and corresponding hydrodynamic circuit model of the device used for threshold bypass pressure measurement is depicted in figure $\mathbf{B A}$ and $\mathbf{B}$. Utilizing this model, the volumetric flowrate through the constriction geometry

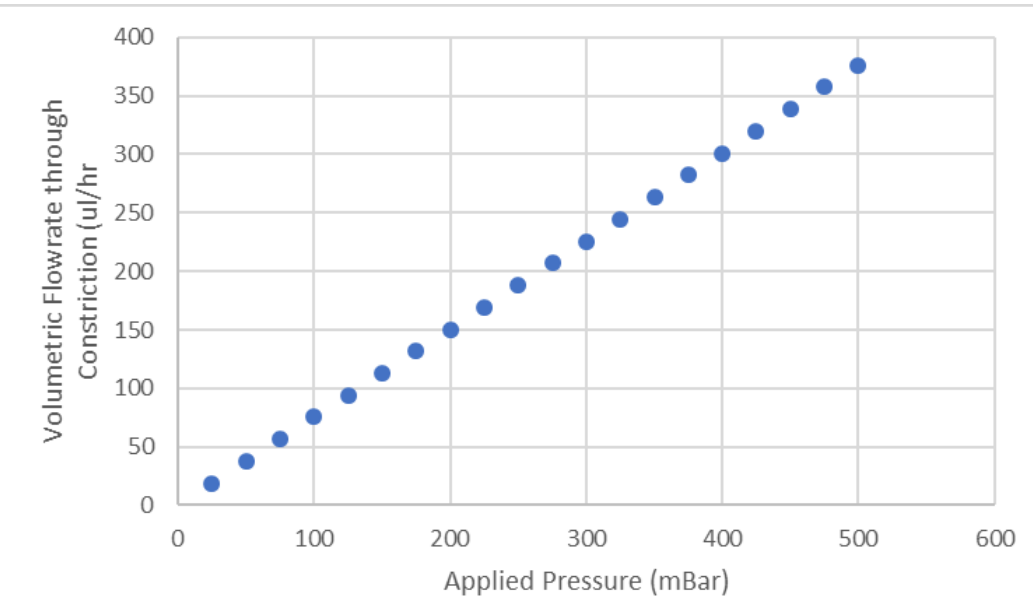
was calculated for varying Figure 2: Volumetric flowrate through the single channel device versus the pressure levels. Hydraulic experimentally measured applied pressure.

resistance values for the constriction geometry (equation 7 and 6), the tubing inserted at the inlet and outlet of the device equation (equation 4), and the channels leading to and from the constrictions (equation 5) were determined using the outlined in section 5 of the supporting information. Since a pneumatic pressure source was used to apply a constant pressure to the device it was modeled as a voltage source. During device operation, a range of pressures from 25 to 500 mbar were applied at the device inlet. The corresponding applied pressures and volumetric flowrates through the device are provided in figure $\mathbf{2}$. The flowrates calculated from this analysis were then used to achieve separation in the filtration device. 


\section{Size and deformability based separation of cellular aggregates}

We seek to remove acinar tissue populations that broadly exhibit smaller sizes and higher deformability levels relative to islets, so that the separation strategy causes minimal stress on the collected islets, thereby retaining their viability and angiogenic potential after transplantation. Dead-end microfluidic filtration platforms[26],[46], wherein large and rigid particles are selectively trapped under pressure driven flow across a micro-scale pore, have been widely applied in prior work for this purpose. However, this method suffers from clogging-induced alterations to the hydrodynamic resistance across pores, thereby increasing the applied pressure over time and resulting in poor selection purity[50], as well as causing non-reversible adsorption of the particle to the filtration microstructure to result in sample loss. Cross-flow filtration attempts to alleviate these issues by using a continuous flow tangential to the micro-scale pores, so that most particles pass in the tangential direction except for small particles, while collecting large particles in the tangential direction[49],[50],[51]. However, while this method is effective for size-based separation, the tangential flow is unable to maintain a consistent level of deformation on the trapped particle, because the pressure differential is significantly altered upon particle trapping in the constriction region. This is especially problematic with multi-cell aggregates that exhibit wide size and deformability distributions, thereby causing them to require longer time intervals for deformation under a consistent pressure differential. Hence, in order to reduce sample loss and enhance selection purity, we

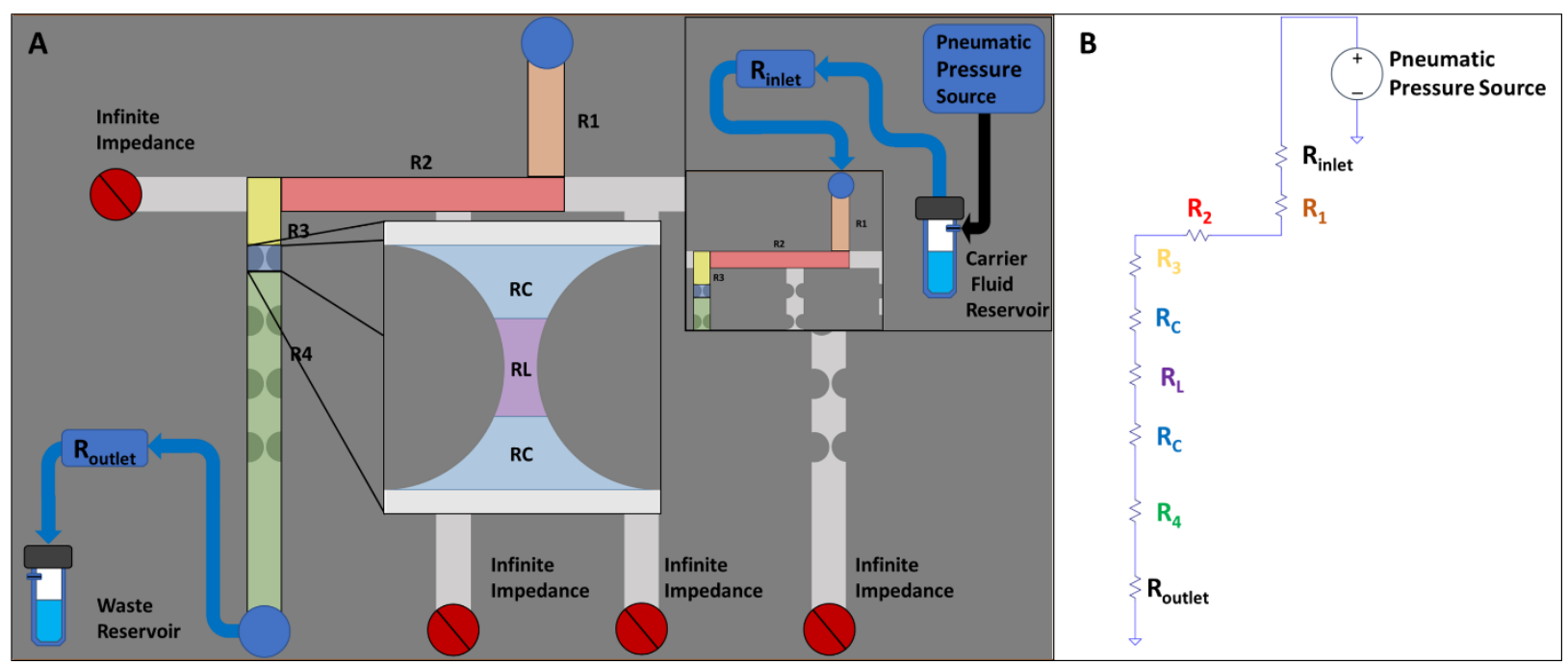

Figure 3: Schematic of the single channel threshold bypass pressure measurement device B: Hydraulic circuit model of $A$ 
use a two-cycle separation device, wherein two-way piezoelectric switches at each outlet allow for rapid reconfiguration of the hydrodynamic resistance of the flow pathways at a set of bifurcating junctions. As per Fig. 4a, using a low resistance setting at constricting channels of the bifurcating junction versus a high resistance setting at the main outlet, the separation cycle allows deformable acinar particles to pass through to the waste port under pressure driven flow, while sequentially trapping individual islets at the junction of bifurcating constricting channels. In particular, multi-cell aggregates are focused at the channel wall of the bifurcating junction due to the high speed balancing flow ( $W_{a}$ determined by ratio of the respective flow rates at sample to balancing flow) and the hydrodynamic resistance at the main outlet versus the bifurcating channel (which depends on $W_{s}$ ) is set to enable the selective deformation of aggregates below a desired size range and up to a particular deformability level (as indicated by $W_{1}$ ), based on the measured threshold bypass pressure from Section 2.1. At a critical occlusion level across the array of bifurcating channels, the hydrodynamic flow resistances are switched as per Fig. $\mathbf{4 b}$ to enable the tangential flow-based collection cycle, wherein islets that are trapped at the bifurcating junctions are rapidly selected for collection through the main outlet. As per Fig. $\mathbf{4 c}$, the arrayed device consists of a main channel ( $400 \mu \mathrm{m}$ width by $350 \mu \mathrm{m}$ in height) with a series of parallel bifurcating channels ( $80 \mu \mathrm{m}$ width by $350 \mu \mathrm{m}$ in height), with each preceded by a focusing flow channels (100 $\mu \mathrm{m}$ width by $350 \mu \mathrm{m}$ in height). The main channel terminates to an outlet for sample collection, while the bifurcating channels share a common outlet for waste removal. The high flow rate tangential focusing flows prior to each

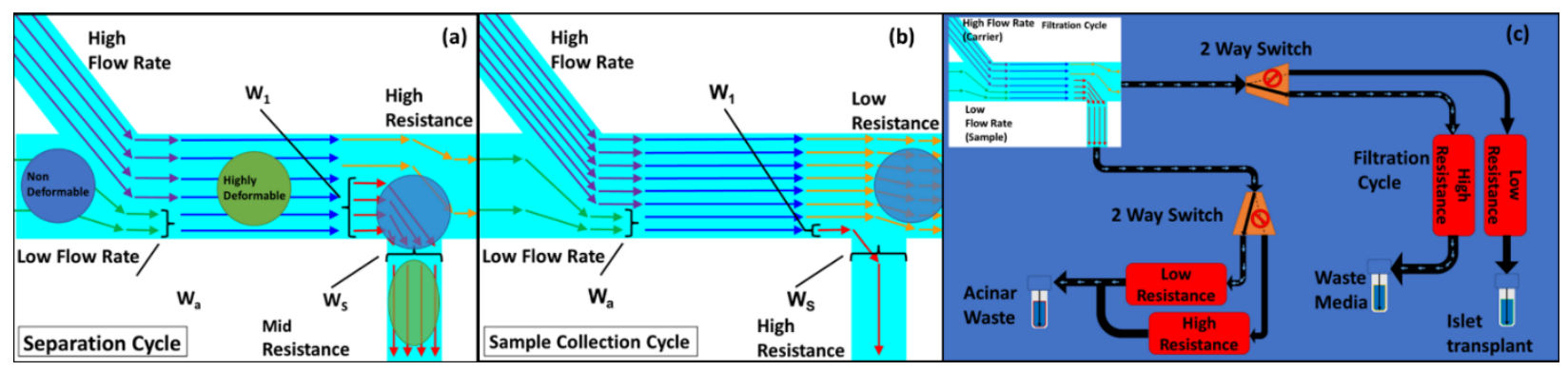

Figure 4: Microfluidic tangential flow design with a high-speed balancing flow to place multi-cell aggregates at the channel wall of a bifurcating junction for: (a) separation cycle wherein a high added resistance at the main outlet is used to direct the flow towards the bifurcating channel for removing smaller and more deformable acinar particles; (b) collection cycle wherein flow resistance of the main outlet is lowered versus that of the bifurcating channel for periodic collection of rigid islet particles; (c) overall device and fluidic connections to enable rapid switching between the separation and collection modes by using a set of flow multiplexers, with pre-programed time intervals for each cycle that are determined based on occlusion levels due to islets at the bifurcating junctions; volumetric flow rate and hydrodynamic resistances are adjusted across the length of the device array using an equivalent fluidic circuit model to ensure consistent pressure drops across each bifurcating channel that just exceed the threshold bypass pressure for acinar deformation while being insufficient for islet passage. 
bifurcating channel are optimized through an equivalent flow circuit model to ensure maintenance of a relatively uniform pressure drop over each channel (Fig. 4d), independent of distance from the inlet. This allows for a rapid, backflow-free method to manipulate flow within the microfluidic device, so that the device can rapidly switch between the separation cycle and the following sample collection cycle to enable the periodic isolation of islets that have been subject to minimal stress.

\section{Design criteria for Separation Device}

In order to achieve continuous deformability-based separation of acinar tissue populations from an islet sample a multichannel continuous two key design criteria of the multichannel continuous separation device of Fig. 4 must be calibrated based on those in the single-channel batch mode device for the applied pressures shown to differentiate between islet and acinar tissue. (Fig. 5). These are: (1): the pressure differential across particles at

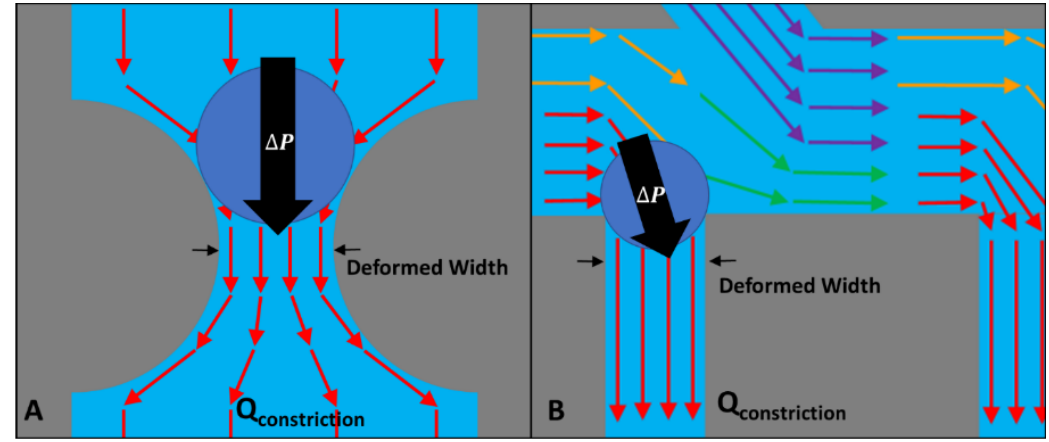

the respective constriction geometry must be roughly equivalent in both devices; (2): the degree of deformation

Figure 5: Schematic of the constriction geometry in the batch single channel threshold bypass pressure B: Schematic of the constriction geometry in the multichannel continuous flow separation device. In order to maintain an equivalent pressure differential across sample particles, the cross sectional area of the channel and the volumetric flow rate through the channel correspond to those of the single channel device.

necessary to bypass the respective constriction must be the same in both devices. The second criterion can easily be achieved by ensuring equal cross sectional areas for the respective constriction geometries within both device-types. In order to achieve the first design criterion, it is important to consider the degree of occlusion of sample particles at the constriction during its selection at the bifurcating point (Fig. 4). As was explored by Kuo et al., the pressure drop over a particle occluding a pore is strongly dependent on the degree of occlusion.[49] For complete occlusion (i.e. clogs), the pressure exerted on the particle is equal to the pressure differential across the entire pore, since the clogging particle prevents all fluid flow through the pore, thereby leading to a strong build-up of pressure on one side of the particle. On the 
other hand, in cases of only partial occlusion, wherein the carrier fluid may still flow around the particle, far lower pressure differentials are achieved, since drag forces from the carrier fluid continue to exert pressure on the particle at the partially occluded constriction. For cases of $<75 \%$ occlusion, the pressure differentials rise only weakly with occlusion (within one or two orders of magnitude), following a similar relationship to the pressure imparted by a carrier fluid on an unobstructed particle.

$$
\Delta P=\frac{3 \mu v}{R}
$$

Equation: 8

Where $\Delta P$ is the pressure differential across the particle, $R$ is the radius of the particle, and $v$ is the relative velocity of the carrier fluid. In this way, for two constrictions with identical cross sectional area, the pressure applied to a particle will be equivalent, as long as the volumetric flowrate through both constrictions is the same (ensuring similar fluid velocity). For this reason, to simulate the pressure deferential used to achieve deformation in the single channel batch measurement device (Fig. 1), the multi-channel filtration device (Fig. 2) was designed to achieve similar flowrates through similarly sized $(80 \mu \mathrm{m})$ bifurcating channels to those used in the single channel device. In this manner, by matching the flow rates through these bifurcating channels to those necessary to primarily deform acinar tissue and not islet tissue, isolation of the rigid islets was achieved.

\section{Hydraulic circuit model optimization of channel dimensions}

Multiple parallel bifurcating constriction channels were used in the separation device to allow for the sequential trapping of multiple islets at each bifurcating point, prior to their removal during the collection mode. In order to maintain consistent flowrate through each bifurcating channel along the entire length of the main channel, a circuit model is used to optimize device design. As outlined in section S2 of the supporting information, the cross-sectional area of each bifurcating channel and the volumetric flowrate through each bifurcating channel (Fig. 4) were set to match that of the single constriction in the batch measurement device (Fig. 1). As such, maintaining a relatively similar flowrate through each bifurcating independent of its position along the main channel is important to achieve a consistent 
pressure drop across particles at the channel opening. The layout and hydrodynamic circuit model for an N4 (i.e. a device having 4 bifurcating channels and preceded by 4 focusing flows) is depicted in figure 6 a and $b$. The hydrodynamic resistances of each component were determined using equation from the section above. The two independent pumping sources were constant flow rate syringe pumps and therefore modeled using current sources. In order to maintain a similar flowrate through each channel, the hydrodynamic resistance of each bifurcating channel must be designed to be several orders of magnitude higher than that of the main channel. This may be understood using a simplified version of the circuit for two bifurcating channels as depicted in figure $6 \mathrm{c}$. Here the two focusing flows and the fluid flow from upstream of the device are represented by individual current sources whereas the flowrate through the bifurcating channels will correlate to the current through the Rb1 and Rb2 resistors. Using superposition in conjunction with the current divider equation the difference in flowrate between the first and second bifurcating channel may be written as follows.

$$
\Delta Q_{1-2}=\frac{2 R_{m}\left(Q_{d s}+Q_{f}\right)}{\left(2 R_{b}+2 R_{m}\right)}
$$

Equation: 9

Where $R_{m}$ is the hydrodynamic resistance of the main channel components, $R_{b}$ is the hydrodynamic resistance of the bifurcating channels $Q_{d s}$ is the down-stream flowrate, and $Q_{f}$ is the focusing flow-rate. This shows that the difference between the flowrates of these two channels may be limited by ensuring the hydrodynamic resistance of the main channel $(\mathrm{Rm})$ is much lower than that of the bifurcating channels. As equation $\mathrm{S} 6$ demonstrates this may be done by changing the channel geometries. In this case, as the width and height of the channels is determined by the application, the length of the channels has been increased. In this way variation of the bifurcating channel flowrates was limited to less the $1.2 \%$ for $\mathrm{N} 4$ device depicted in figure $6 \mathrm{~A}$. 


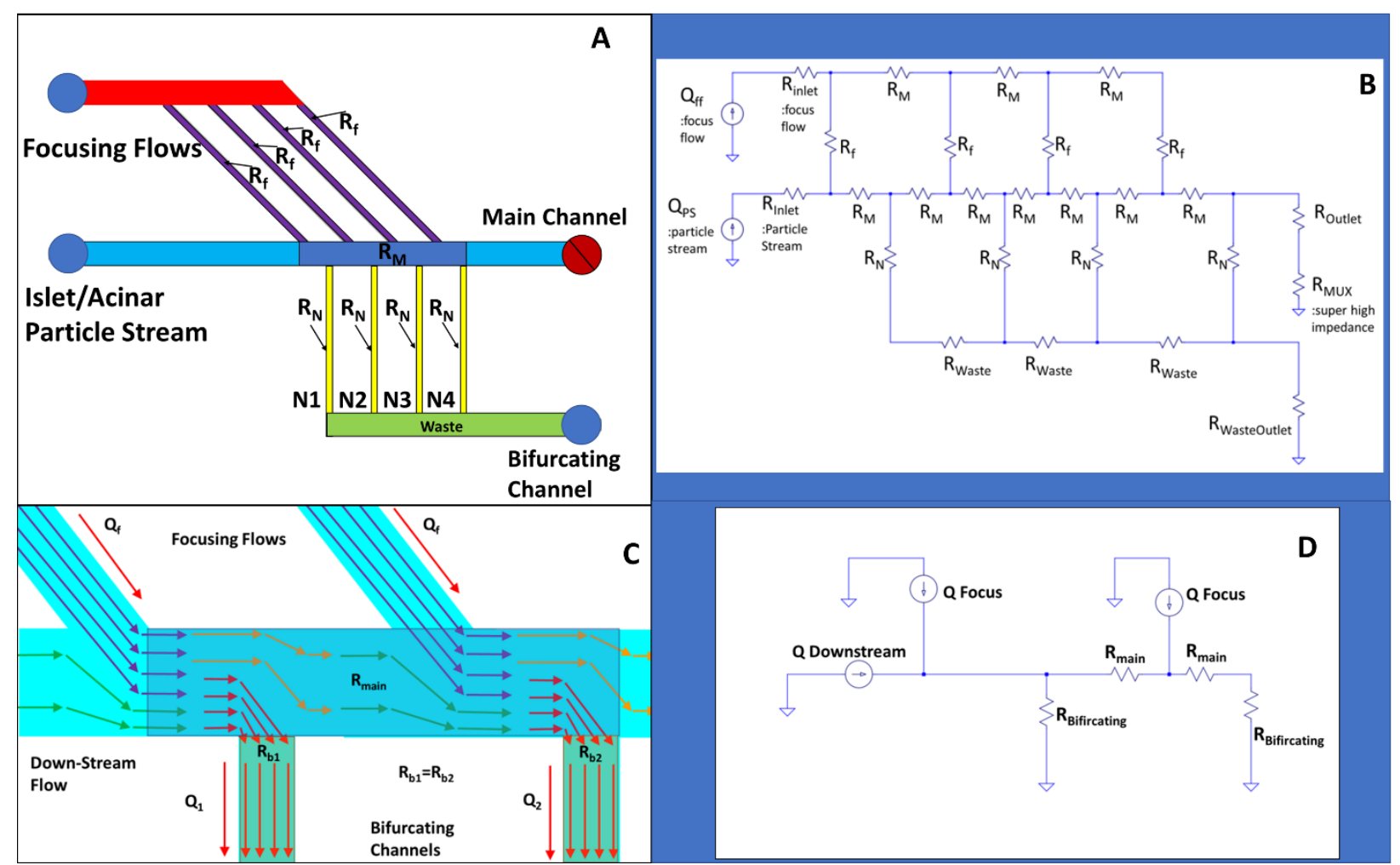

Figure 6: : A schematic of an N4 (4 bifurcating channels) continuous flow separation device B: Hydraulic circuit model of the N4 device pictured in A. C: a simplified schematic of a $2 \mathrm{~N}$ device. D: simplified Hydraulic circuit

\section{Need for focusing flows in separation device}

For this application, the goal of the continuous flow separation device is to isolate and collect larger and less deformable particles (pancreatic islets) while removing smaller more deformable particles (acini tissue) to a waste port. Unlike the cases wherein the smaller or more deformable particles are the sample of value, we seek to collect the larger and more rigid tissue. In order to achieve a high purity final sample it is vital all injected material interact with deformation through a bifurcating channel. In order to ensure this focusing flows prior to each bifurcating channel are required to ensure all material introduced by the particle stream will interact with the first available free (with no trapped particle) channel. When fluid flow through a channel interacts with a bifurcating channel the flow is split between the main channel and the bifurcating channel as is described by the current divider equation. In cases where the fluid flow through these channels is laminar the width of fluid removed down the bifurcating channel is related to ratio of the

\begin{tabular}{|c|c|c|}
\hline $\mathbf{N}$ & Wps (um) & Ws (um) \\
\hline 1 & 228.5714 & 226.2181 \\
\hline 2 & 201.8138 & 262.758 \\
\hline 3 & 163.6774 & 316.0964 \\
\hline 4 & 104.5731 & 400 \\
\hline
\end{tabular}


flowrate through the main channel and the bifurcation channel as is demonstrated in figure 7. Using a rough 1 dimensional model this width may be approximated as.

$$
\frac{Q_{2}}{Q_{1}+Q_{2}}=\frac{W_{p s}}{W_{t o t}}: \frac{Q_{4}}{Q_{3}+Q_{4}}=\frac{W_{s}}{W_{t o t}}
$$

Equation: 10

Where $Q_{1}$ is the flowrate of the focusing flow, $Q_{2}$ is the flowrate of the particle stream, $Q_{3}$ is the flowrate through the main cannel after the bifurcating channel, $Q_{4}$ is the flowrate down the bifurcating channel, $W_{p s}$ is the focused width of the focus flow, $W_{s}$ is the selection width of the bifurcating channel, and $W_{\text {tot }}$ is the total width of the channel.[40] As particles carried by laminar flow tend to remain in the flow stream incident to their center of gravity, only particles whose center of gravity is within the width of removed fluid will interact with the bifurcating channel. For typical separation devices utilizing many bifurcating channels branching from the same main channel the decrease in flowrate along the main channel results in substantial variation in this selection width. Depending on a partices position within the width of the carrier stream this limits the selection points it may interact with, potentially leading to sample loss in cases where all available bifurcating channels have been filled by trapped low deformability particles. In order to allow all particles to interact with any of the bifurcating channels focusing flows prior to each bifurcating channel are used. This both focuses the particle stream and helps to maintain more consistent selection widths throughout the length of the device. F or and N 4 device, in order to maintain a selection width of at least 200 um (50\% of the main channel and

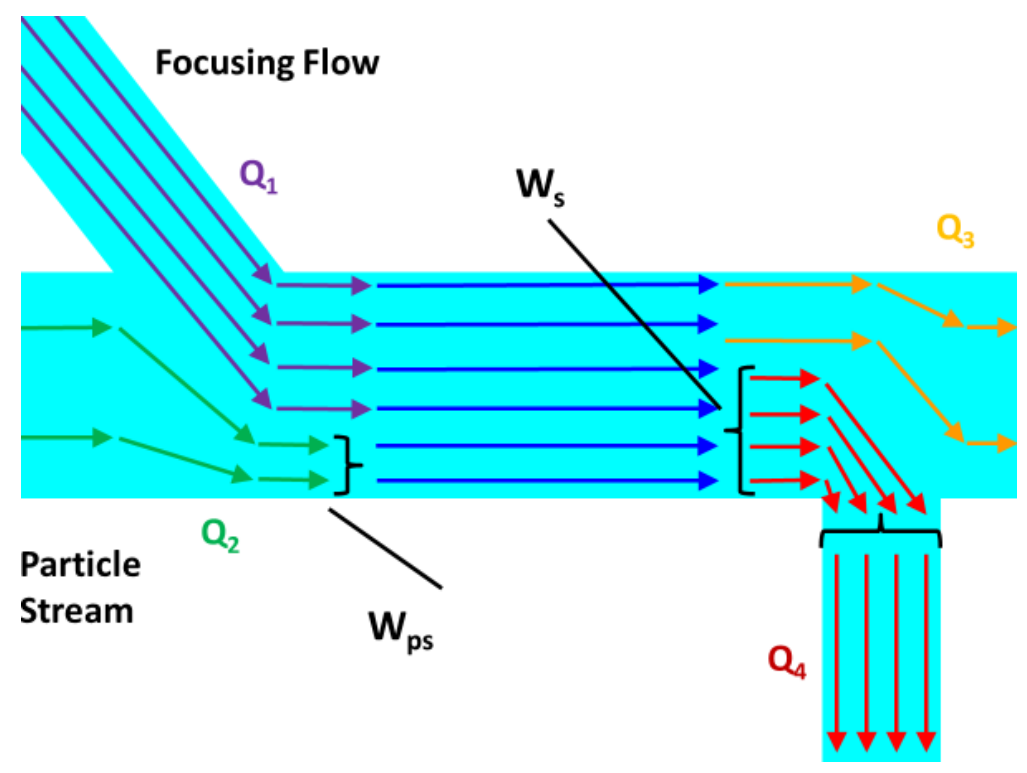

Figure 7: Particles carried by the carrier fluid will be focused to a position within the particle stream width $\left(W_{p s}\right)$ as determined by= the ratio of the volumetric flowrate $Q_{1}$ versus $Q_{2}$. Particles within the selection width $\left(W_{s}\right)$ will interact with the bifurcating channel as determined by the ratio of $Q_{3}$ to $Q_{4}$. By ensuring $W_{p s}<W_{s}$ all particles within the main channel will interact with the bifircating channel 
$100 \%$ of the particle stream) the ratio of the flowrates delivered by the pumping mechanism driving the particle flow (Isf) and the focusing flows (If) is $1 / 2.1949$. The width of the particle stream after focusing and the selection width for each bifurcating channel is given in table 1. 


\section{Aim Two Background: Electrokinetic preconcentration and electrochemical detection within a nanoslit platform}

\section{Dielectrophoresis}

Electrokinetic techniques, based on the electro-physiological properties of a bioparticle, are a versatile and selective range of methods to manipulate micro- and nanoscale bioparticle in a micro/nanofluidic system. One of the most versatile electrokinetic methods utilizes dielectrophoresis (DEP), a phenomena, which exerts a deterministic force on polarized particles suspended in an aqueous medium when placed under a spatially non-uniform electric field. This force is directed either towards or away from an engineered high field point based upon the relationship of the frequency dependent polarization of the target particle and its surrounding medium. For a spherical particle under a non-uniform electric field, the time averaged DEP force can be described by the flowing equation

$$
F_{\text {DEP }}=2 \pi \varepsilon_{\mathrm{m}} R^{3} K c m(E \nabla \mathrm{E})
$$

\section{Equation: 11}

Where $\mathrm{R}$ is the radius of the spherical particle, $\mathrm{E}$ is the magnitude of the electric field, $\mathrm{DE}$ is the gradient of the electric field, $\varepsilon_{\mathrm{m}}$ is the absolute permittivity of the medium, and $\mathrm{Kcm}$ is the Clausius-Mossotti factor, which is a relationship comparing the polarizability of the particle to the suspending medium. The Kcm is given by

$$
K c m=\left(\frac{\varepsilon_{p}^{*}(\omega)-\varepsilon_{m}^{*}(\omega)}{2 \varepsilon_{m}^{*}(\omega)+\varepsilon_{p}^{*}(\omega)}\right)
$$

\section{Equation: 12}

Where $\varepsilon_{p}^{*}(\omega)$ and $\varepsilon_{m}^{*}(\omega)$ are the frequency dependent complex permittivity of the particle and medium respectively. As equation 3 and 4 demonstrate, while the magnitude of the DEP force is determined by the size of the particle $(R)$, magnitude and gradient of the electric field $(E \nabla \mathrm{E})$, and the Clausius-Mossotti 
factor $(\mathrm{Kcm})$, the direction of the electric field is determined solely by the $\mathrm{Kcm}$. When the particle polarizes more strongly than the medium $\left(\varepsilon_{p}^{*}(\omega)>\varepsilon_{m}^{*}(\omega)\right)$ the particle is translated towards high field points in a phenomena known as positive DEP ( $\mathrm{pDEP})$. When the reverse is true $\left(\varepsilon_{p}^{*}(\omega)<\varepsilon_{m}^{*}(\omega)\right)$ the Kcm is negative and the particle is translated away from the high field point in a phenomena known as negative DEP (nDEP). As this relationship is both frequency dependent and varies for different sample particles based on their individual electrophysiology the direction and magnitude of the applied force may be tailored to selectively translate target particles to specific trapping regions, thereby achieving sample enrichment.

There are several methods to produce the high field nonuniformities required for DEP enrichment. A common method is through the use of planar deposited metal electrodes spaced within a few microns of each other (MDEP)[52]. Another method, which is easily integrated with a sensing scheme, is insulator DEP (iDEP). [5, 7, 53-56] This method utilizes insulating microstructures that act as field lenses to generate spatial field nonuniformities. A common permutation of this method uses triangular constrictions in a microfluidic channel as is demonstrated in figure 8. In this method, high field points are created at the constriction tips and the field magnitude and gradient is affected by a range of parameters including, constriction gap width, maximum channel width, and the slope of the constrictions. Direct use

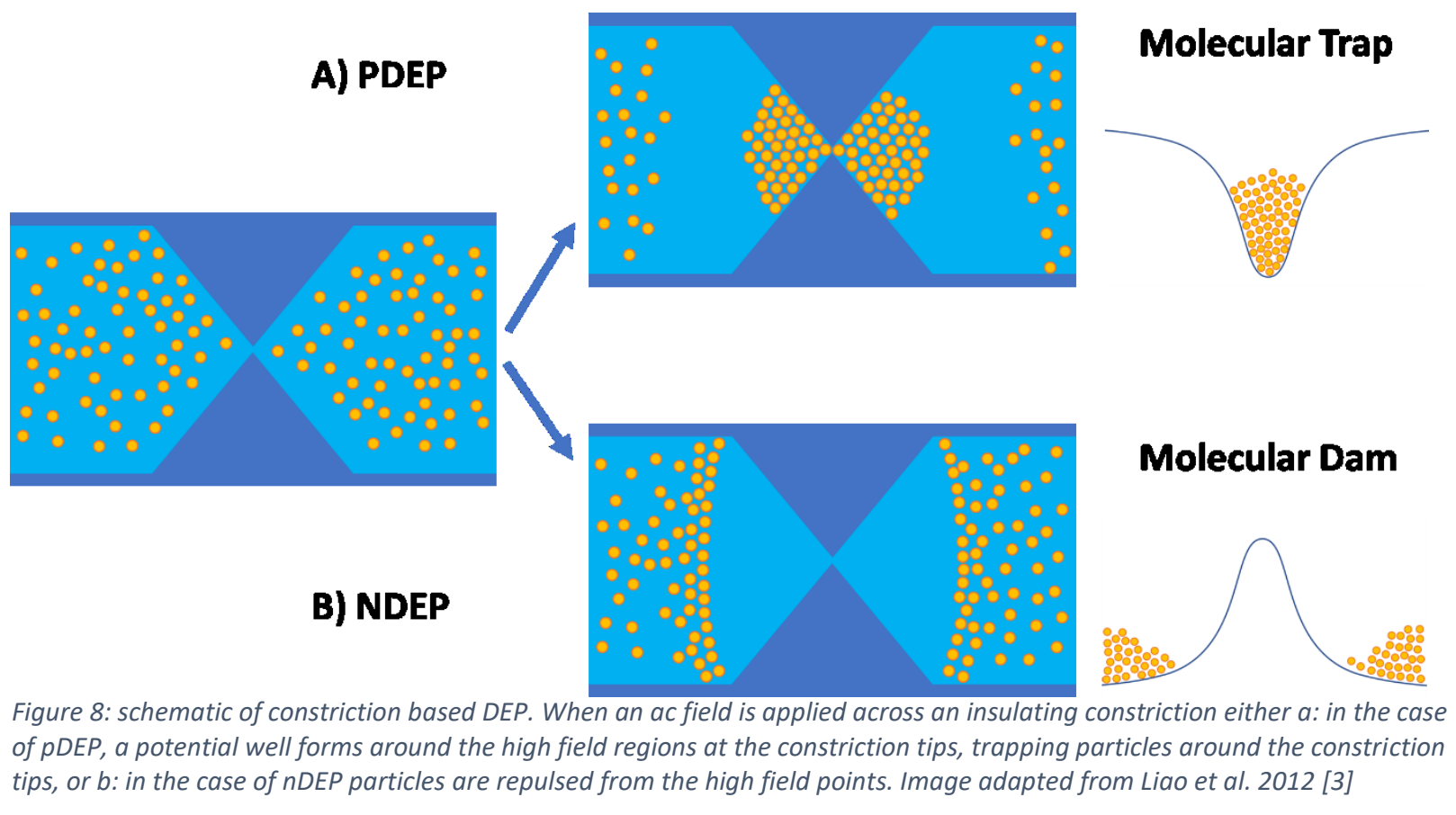


of DEP trapping for analyte preconcentration in a nanochannel, however, suffers from a severe drawback. Analyte enrichment due to DEP preconcentration occurs only over a severely limited region near the microstructure field lenses. This makes alignment and integration with sensor architecture to be extremely difficult. This drawback may be alleviated however by utilizing nDEP as a component in an electrokinetic force balance approach to preconcentration.

\section{Negative Dielectrophoretic (nDEP) Dam Method}

Negative dielectrophoretic damming refers to the use of an electrokinetic force balance of nDEP under AC fields versus electrokinetics under DC fields to trap biomarkers and thereby achieve high degrees of sample enrichment. [1-4, 57] In order to utilize this scheme, an array of insulating micro- or nanoconstrictions are integrated within a nanoslit platform and are used as force lenses to create field non uniformities in an AC electric field applied over the nanoslit. The frequency of the AC field is chosen such that the target analyte undergoes nDEP and is thus translated away from the high field points at the

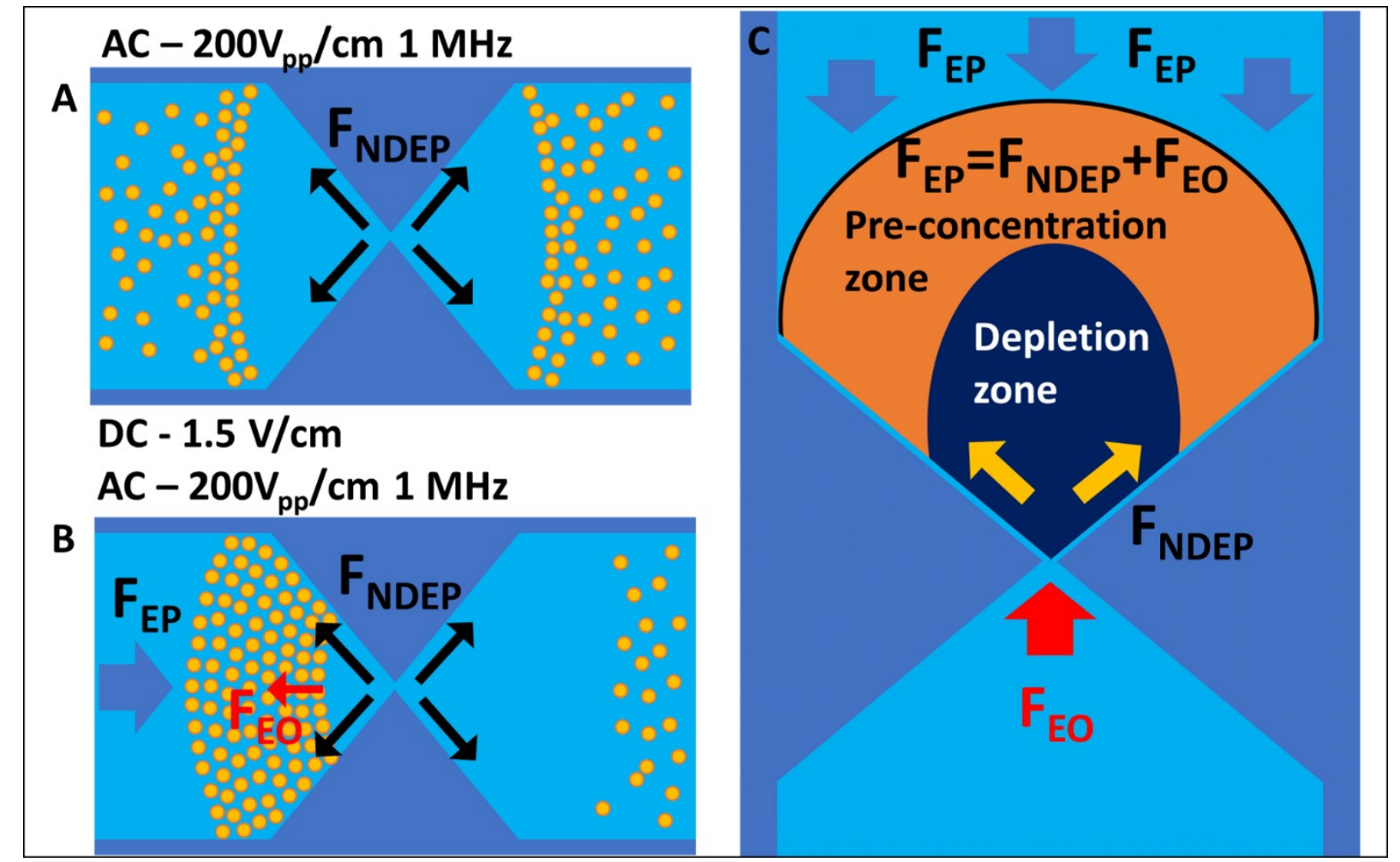

Figure 9: : Schematic of preconcentration due to an electrokinetic force balance. A: trapping utilizing only nDEP. B: The application of a low magnitude DC offset results in a balance between the electrokinetic forces of $n D E P$ and Electro Osmosis with Electrophoresis resulting in significant sample preconcentration. C: the formation of a depletion and preconcentration zones. The depletion zone forms near the constriction tips due to nDEP force. image adapted from Lia et al. 2011 [4] 
tips of the nanoconstrictions. Concurrent to this, a low magnitude DC offset is added to the AC field to achieve net transport of the analyte through the nanoslit via electrophoresis. Within the nanoslit, on one side of the micro/nanoconstriction (as determined by the charge of the analyte) the force from nDEP and EP oppose one another and a force balance is achieved such that.

$$
F_{E P}=F_{E O}+F_{n D E P}
$$

\section{Equation: 13}

This balance of forces creates a potential well which traps analyte delivered to the region by electrophoresis causing accumulation as is depicted in figure $\mathbf{9}$. The resulting accumulation occurs over a significant area of the nanoslit allowing for easy integration with sensing elements as it prevents unwanted interactions between the sensor and field lens architecture. $[1,57,58]$ In order to achieve analyte accumulation utilizing the nDEP damming method, a high degree of nDEP must be exerted on the sample. This criteria is made difficult when preconcentrating typical nanoscale molecular or nanocolloidal analyte particles. As has been previously described in equation $11, \mathrm{nDEP}$ force scales drastically with the size of the suspended particle. In order to overcome this issue it is necessary to significantly enhance both the field magnitude and gradient. 


\section{Chapter 2: Methods}

\section{Aim One Methods: Deformability based separation of islets}

\section{Human pancreas digestion and density gradient purification}

The overall process for generating the samples is indicated schematically in Fig. 10. In order to release the pancreatic islets (Fig. 10a) and surrounding acinar tissue from the donor pancreas, enzyme perfusion with collagenase is introduced through the pancreatic duct to dissemble the donor organ via digestion (Fig. 10b). After perfusion, the organ is loaded into a digestion chamber containing metal ball bearings for enabling vigorous agitation to cause the fragmentation of the organ into successively smaller aggregates of endocrine and exocrine tissue. A continuous flow through the digestion chamber carries aggregates of similar volume to pancreatic islets through a screen out of the digestion chamber into a collection flask, wherein the enzymatic digestion of the collected aggregates is quenched through cooling

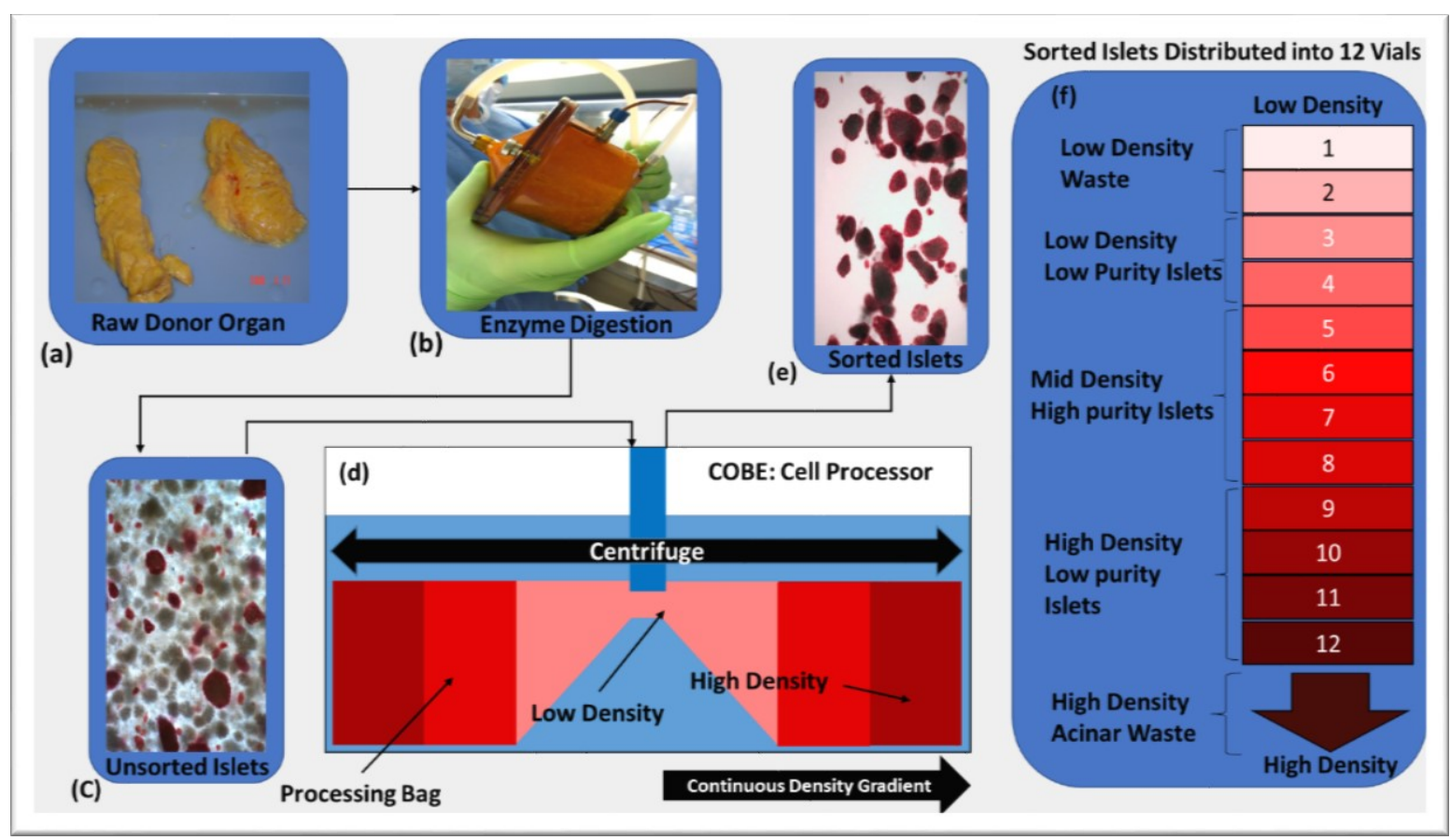

Figure 10: Schematic of the sample preparation process: (a) raw donor organs; (b) enzyme digestion; (c) sample mixture of islets (stained for insulin) and acinar tissue; (d) COBE cell processor for density based separation; (e) sorted islets from a mixture of samples from centrifuged fractions ( $f$ ). 
and dilution. As a result, the donor organ is reduced to slurry of islet and acinar aggregates of similar size (Fig. 10c).

An additional purification step is required to meet the transplantation needs, since the slurry has an islet purity of $1-2 \%$. For this purpose, the digested sample is purified by a density gradient separation method using a COBE 2991 cell processor (Fig. 10d). Herein, a continuous density gradient media is produced and loaded into COBE cell processor, so that the density of the media increases laterally from the center of the process bag. The tissue sample is loaded into the center of the process bag, which is then spun at $1500 \mathrm{rpm}$ for 30 minutes. During this time, the centrifugal force separates the digested aggregates along the density gradient, with the denser acinar material moving to the outer regions of the process bag and the lower density islet material remaining towards the center of the process bag. The contents at the central portion of the process bag are then separated into 12 fractions, each allocated into separate vials of $10 \mathrm{~mL}$. The remaining volume in the process bag composed of larger islet and acinar tissue pieces is dioscarded. The tissue samples from the collected fractions (Fig. 10e) are then washed and incubated overnight to allow the islets to recover from the digestion process. During the wash step, each of the 12 collected fractions (Fig. 10f) is centrifuged and the volume of the plug is measured to determine the packed tissue volume of each fraction. After resuspension, a sample is taken from each collected fraction to estimate its islet purity, by comparing the overall aggregate area of the acinar and islet populations using phase contrast microscopy. By comparing the packed tissue volume to the fractional purity, the islet and acinar tissue volumes are estimated. Collected fractions of similar purity are then combined to produce three plates of varying purity levels: high (fractions above $80 \%$ purity); medium (80\%-50\%); and low (50\%-30\%). These plates are incubated overnight for use within the described studies, while fractions of purity below $30 \%$ are discarded.

\section{Mouse Islet isolation}

These islets were isolated from mouse pancreases using a modified common bile duct (CBD) profusion protocol. Collagenase enzyme solution (Collagenase P, Roche, Indianapolis, IN, USA, enzyme at $1.4 \mathrm{mg} / \mathrm{mL}$ in a modified Hank's Balanced Salt Solution, Invitrogen, Carlsbad, CA, USA) is first injected into 
the pancreas via the CBD. The pancreas is then excised and submerged in modified $37^{\circ} \mathrm{C}$ Hank's Balanced Salt Solution for 8-11 $\mathrm{min}$. After digestion a density gradient solution $(1.100 \mathrm{~g} / \mathrm{mL})$ is used to separate the denser acinar tissue from the pancreatic islets which are then suspended in a growth media. Often the resulting sample is allowed to culture overnight in an incubator prior to separation to allow the pancreatic islets a chance to recover from the isolation protocol.

\section{Viability and insulin production assays}

The viability of pancreatic islets is determined through the use of a Propidium lodide (PI) florescent stain. This fluorescent dye targets the nuclei of non-viable cells with damaged (highly permeable) cell membranes. As islets are cell aggregates viability is lost when a significant portion of the aggregated cells have lost viability (above 70\%).

Pancreatic Islets are differentiated from acinar tissue fragments through the use of a dithizone (DTZ) Stain. This dye stains zinc containing insulin granules and effectively detects insulin production in Islets staining them red.

\section{Experimental setup: microfluidic threshold bypass pressure measurement}

The overall layout of the chip and fluidic control system for enabling threshold bypass pressure measurements is shown in Fig. 1e, with expanded view of each section of interest within Fig. 1f and 1g. The device is comprised of an inverted T-intersection that is used to select single aggregates into each of the four individually addressable branching channels (four shown in schematic) that lead to constrictions of different sizes. After several initial optimizations experiments, we selected the test region comprised of a channel with $400 \mu \mathrm{m}$ width and $350 \mu \mathrm{m}$ height that has a lateral constriction of $80 \mu \mathrm{m}$. To ensure that only a single particle enters the constriction at a time for preventing clogging of the constriction, we use a two-stage batch flow method to select individual sample particles. As per Figure $\mathbf{1} \mathbf{f}$ and $\mathbf{1 g}$, sample aggregates are injected using a syringe pump, which is used to move individual aggregates to the T intersection. Once the aggregate reaches the intersection, the syringe pump is stopped and a pressure driven flow is applied from the perpendicular channel to carry the aggregate towards the constriction of 
interest. After the aggregate has been moved towards the constriction, the pressure is increased by 25 mbar every 40 seconds, starting at 25 mbar, so that the aggregate deforms through the constriction. This process is recorded by an inverted microscope (Zeiss Observer Z1) to note the pressure at which the tested aggregate passes through the constriction. Following passage of the aggregate, a high pressure is applied to fully remove the deformed aggregate from the device to prevent backend clogging induced errors to pressure drop over the device. In the design of Fig. 1, the threshold bypass pressure can be measured simultaneously for four aggregates, which is repeated for a wide range of aggregate sizes that are measured by their pre-deformed area.

\section{Experimental setup: Microfluidic separation of islets}

A low purity sample containing a mixture of islet and acinar aggregates, which is either the sample drawn prior to ( $<20 \%$ purity) or the one following the density gradient separation step (60\% purity) is used in the microfluidic separation studies. As per Fig. 4d, the respective sample is introduced through the main channel inlet and the high flow rate balancing flow is applied to drive sample aggregates towards bifurcating channels. The multiplexer is programmed for a series of separation and collection modes, with their respective time intervals set based on starting islet levels within the sample (for instance: samples with higher islet levels require successively lesser separation time in between collection cycles). During the separation mode, the high flow rate directed from the inlet and balancing flows towards the bifurcating channels causes the smaller sized and higher deformability acinar aggregates to be removed through to the waste ports, while also trapping of a critical number of rigid islets at the bifurcating junction, with the occlusion detected by flow rate alterations. At a critical occlusion level, the multiplexer switches flow resistances to the collection mode, wherein the trapped islets at the bifurcating junctions are carried out through the main outlet port. Following collection, the fluidic control system is reconfigured for the separation mode and this process is repeated multiple times until the entire input sample is passed through the device. The flowrates of the main carrier and balancing flows required to generate specific pressure drops over the branching channels were numerically calculated using an equivalent fluidic circuit analogy method. The flowrates of the balancing flows are chosen to minimize the 
pressure drop between bifurcating channels in the absence of particles to ensure minimal pressure differences between each bifurcating channel. Based on the sample composition of the respective islet versus acinar populations, the time intervals for the separation and collection modes is optimized, such that the separation time between collection cycles is approximately the average time for trapping particles in $<50 \%$ of the branching channels. Typical values ranging from 21 seconds for input samples with an initial purity of $18 \%$, to 79 seconds for input samples with an initial purity of $56 \%$. In this manner, an input sample that includes acinar tissue populations mixed with islets can be purified to enable the continuous collection of a sample with high purity pancreatic islets.

\section{High aspect ratio Microdevice fabrication}

The microfluidic devices utilized in this experiment were fabricated by micro-molding Polydimethylsiloxane (PDMS) into a SU-8 negative photoresist master that is patterned on Silicon using standard photolithography. The thickness of the patterned photoresist master determines the depth of the microfluidic channel, which is set to $350 \mu \mathrm{m}$. To achieve this resist thicknesses, multiple layers of SU8 3050 are spun onto a 4 inch wafer at $1000 \mathrm{rpm}$ and a modified multi-staged soft bake is used. Photolithography is used to pattern the negative photo resist to form a raised $350 \mu \mathrm{m}$ high inverse structure master into which uncured 10:3 PDMS epoxy is poured for micromolding. Following a vacuum degassing step, the mold is allowed to harden overnight on a hot plate. The cured PDMS is then carefully released from the Silicon master leaving the microfluidic design molded on its surface. The PDMS mold is then diced into single devices, followed by drilling of design specific $1 / 16$-th inch inlet and outlet holes with a punch. Following the removal of debris and a low energy oxygen plasma treatment step, the PDMS channel device is bonded to a glass coverslip.

\section{Measurements of islet viability and vascularization}

In order to gauge the angiogenic potential of sorted islets a mouse cornea micropocket assay was conducted by a collaborator, Molly Kelly-Goss. Islets that were sorted by the described microfluidic techniques were compared to the pristine islets that were not passed through the microfluidic device. Viability was determined using propodium iodide staining (Supporting Information) and vascularization 
was studied through transplantation into the corneas of healthy adult mice using the corneal micropocket. As per prior islet transplantation studies into the cornea[10] and the cornea micropocket assay[9], mice were anesthetized with an intraperitoneal injection of ketamine/xylazine/atropine $(60 / 4 / 0.2 \mathrm{mg} / \mathrm{kg}$ body weight) (Zoetis; Kalamazoo, MI / West-Ward; Eatontown, NJ / Lloyd Laboratories; Shenandoah, IA), and a drop of sterile $0.5 \%$ Proparacaine Hydrochloride Ophthalmic solution (Henry Schein Inc; Melville, NY) was added as a topical anesthetic to numb the eye before treatment. A small incision was placed about $1 \mathrm{~mm}$ away from the capillary network surrounding the cornea, parallel to the capillary vessels. Using a sterile surgical blade, an incision was then made in the cornea to make a small pocket between the corneal epithelial layers, toward the capillary network. This was done carefully, so as to not rupture the cornea or poke through to the anterior chamber of the eye. Using a $10 \mu \mathrm{L}$ pipette tip, islets were then transferred from warm and sterile PBS onto the surface of the cornea, just outside the micropocket. The islets were then slid very gently into the micropocket using either a 27-gauge needle or jeweler's forceps. Finally, the micropocket was lightly closed using jeweler's forceps to prevent the islets from regurgitating out of the cornea pocket. Seven days after transplantation, bright field images of corneas under 4X magnification were obtained using a Nikon Digital Sight DS-L2 Camera Controller (Nikon Instruments Inc, Melville, NY; Model 214602) to observe viability of the islets in the cornea micropocket. To harvest tissues for further analysis, anesthetized mice were euthanized in a $\mathrm{CO}_{2}$ chamber and secondarily euthanized with a cervical spine dislocation. Corneas were harvested immediately, then fixed and permeabilized in $100 \%$ acetone for 25 minutes at room temperature. Next, the tissues were blocked with $5 \%$ normal mouse serum and $2 \%$ bovine serum albumin in phosphate buffered saline (PBS). To visualize blood vessels, corneas were superfused with the endothelial cell marker, rat monoclonal anti-mouse cluster of differentiation 31 (CD31) (Dianova; Hamburg, Germany; 1:250 dilution), washed eight times for five minutes in $0.1 \%$ saponin in PBS, and superfused again with goat anti-rat IgG $(\mathrm{H}+\mathrm{L})$ FITC (abcam; Cambridge, UK; 1:400 dilution). Corneas were whole-mounted with coverslips on gelatin-coated slides using a 50:50 glycerol/PBS solution. Digital images of the corneal and islet vasculature were acquired using confocal microscopy (Nikon Instruments Incorporated, Melville, NY; Model TE200-E2; 10X, 20X,and 60X objectives); full-thickness Zstack ( $2 \mu \mathrm{m}$ steps) volume renders were used to capture the entire thickness of the corneal and islet 
vascular networks in each field of view. All surgical procedures were approved by and done in accordance with the Institutional Animal Care and Use Committee at the University of Virginia.

\section{Aim Two Methods: Electrokinetic preconcentration and}

\section{electrochemical detection within a nanoslit platform}

\section{Fabrication of fused silica Nano constrictions within a Nano-slit}

The nanoslit device (Fig. 11) was constructed on fused silica (quartz) using photolithography and

electron beam lithography for resist patterning, followed by reactive ion etching for nanofabrication of channels. First the 3 um deep channels are formed. Piranha solution $(\mathrm{H} 2 \mathrm{SO} 4: \mathrm{H} 2 \mathrm{O} 2=1: 1)$ is first used to clean a 4-inch fused silica wafer. This etch removes organic contaminants and is utilized prior to every patterning and bonding step. After cleaning, $30 \mathrm{~nm}$ thick $\mathrm{Cr}$ is deposited on the wafer. Photo resist is then spun onto the wafer and exposed. The chromium layer is then developed using a $\mathrm{Cr}$ wetetching step. This patterned chrome layer will be used as an etch mask for micro channel etch step. $3-\mu \mathrm{m}$ deep microfluidic channels are then etched by ICP (Inductively Coupled Plasma), with a CHF3/CF4/Ar/O2 mixture at bias/RF power 700/300W for $3 \mathrm{~min}$. The chrome etch mask is then removed and the wafer is diced. Individual chips are then cleaned using piranha etch. The remaining fabrication steps carried out
Photolithography, Cr wet etching and ICP etching to form microchannel
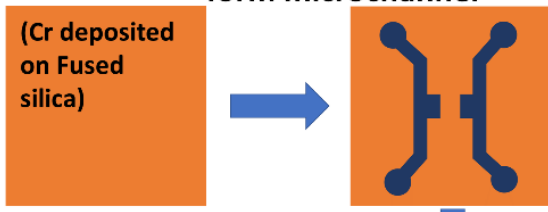

$2^{\text {nd }}$ Photolithography And $\mathrm{Cr}$ wet etching

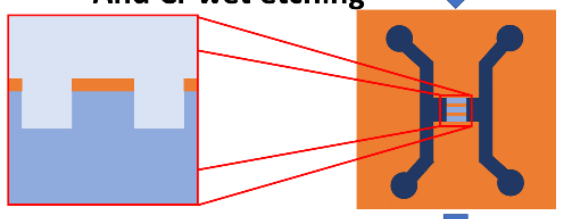

E-Beam lithography, $\mathrm{Cr}$ deposition and Lift-off

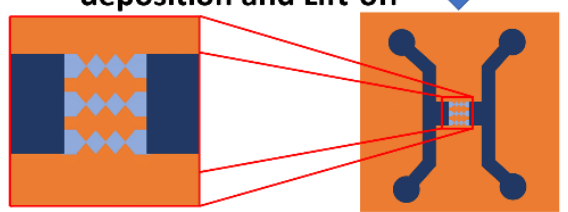

RIE etching to form Nanochannel

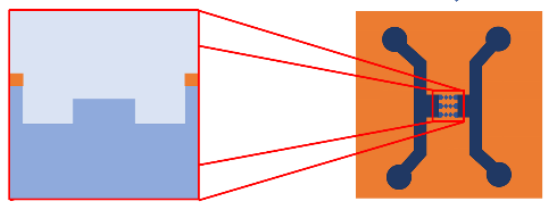

Inlet/outlet drilled by sandblaster and reservoirs glued

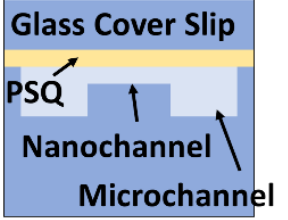

Room-Temperature bonding

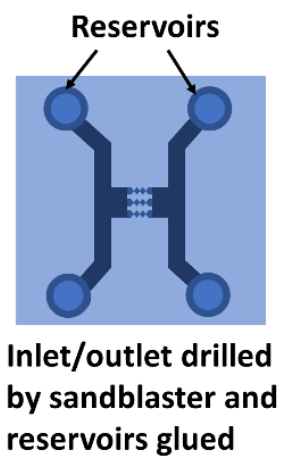


with an individual die. Next the nanoslit and field lens architecture is formed. Photo resist is first spun directly onto the fused silica wafer and patterned using photolithography. Electron beam lithography (EBL) is used as a secondary patterning step to form the tips of the constrictions. $C R$ is then deposited and patterned via a liftoff process. The nanochannel is then etched via RIE (Reactive ion S-3 etching) to a depth of $200 \mathrm{~nm}$ with a CF4/O2 mixture at $20 \mathrm{~W}$ for $10 \mathrm{~min}$.

Inlet and outlet holes are drilled via a sand blaster and the device is assembled prior to use. The channels are encapsulated by a room-temperature and low-pressure PSQ indirect bonding process. First both the fused silica chips and a thin glass coverslip are cleaned using P-etch. A thin PSQ (polysilsesquioxane, Gelest, Morrisville, PA) layer ( $100 \mathrm{~nm}$ thick) is then spin coated onto the glass coverslip and baked for 30 minutes at $240^{\circ} \mathrm{C}$. The coverslip and fused silica chip are then treated using a low energy high pressure oxygen plasma environment. The two treated surfaces are then brought together and pressure is applied allowing the formation of a permanent physical bond. After bonding glass reservoirs are affixed to the inlets/outlets using a UV curing epoxy-glue. Due to the high cost and time consuming nature of this fabrication step Fused silica chips are often reused. The inlet/outlet reservoirs and cover glass are mechanically debonded and adhered PSQ is cleaned off the Fused silica chip using a silicone remover.

\section{Integration of electrochemical detection electrodes}

Following optimization of the field conditions for nDEP enrichment by imaging of fluorescently-labeled NPY and PSA, all subsequent detection was performed by electrochemical methods. Figure 12 a depicts the cross section of the integrated device. Electrochemical detection electrodes were microfabricated on PSQ-coated cover-slip glass (Figure $\mathbf{1 2}$ b), by patterning a standard resist and converting it to glassy carbon (GC) by pyrolysis methods.[59] This was followed by electro-reduction of graphene oxide (ERGO)[60] and electrodeposition of AuNPs at -0.8 $\mathrm{V}$ for $60 \mathrm{~s}$ in $1 \mathrm{mM} \mathrm{HAuCl}_{4}[61]$. Alternate patterned electrodes on the cover-slip function as the reference $\left(\mathrm{Ag} / \mathrm{AgCl}\right.$ prepared by electrodeposition with $\mathrm{Ag}$ and $\mathrm{FeCl}_{3}$ treatment for chloridizing the surface [61]) and counter electrodes (glassy carbon). For both detection schemes, the working 


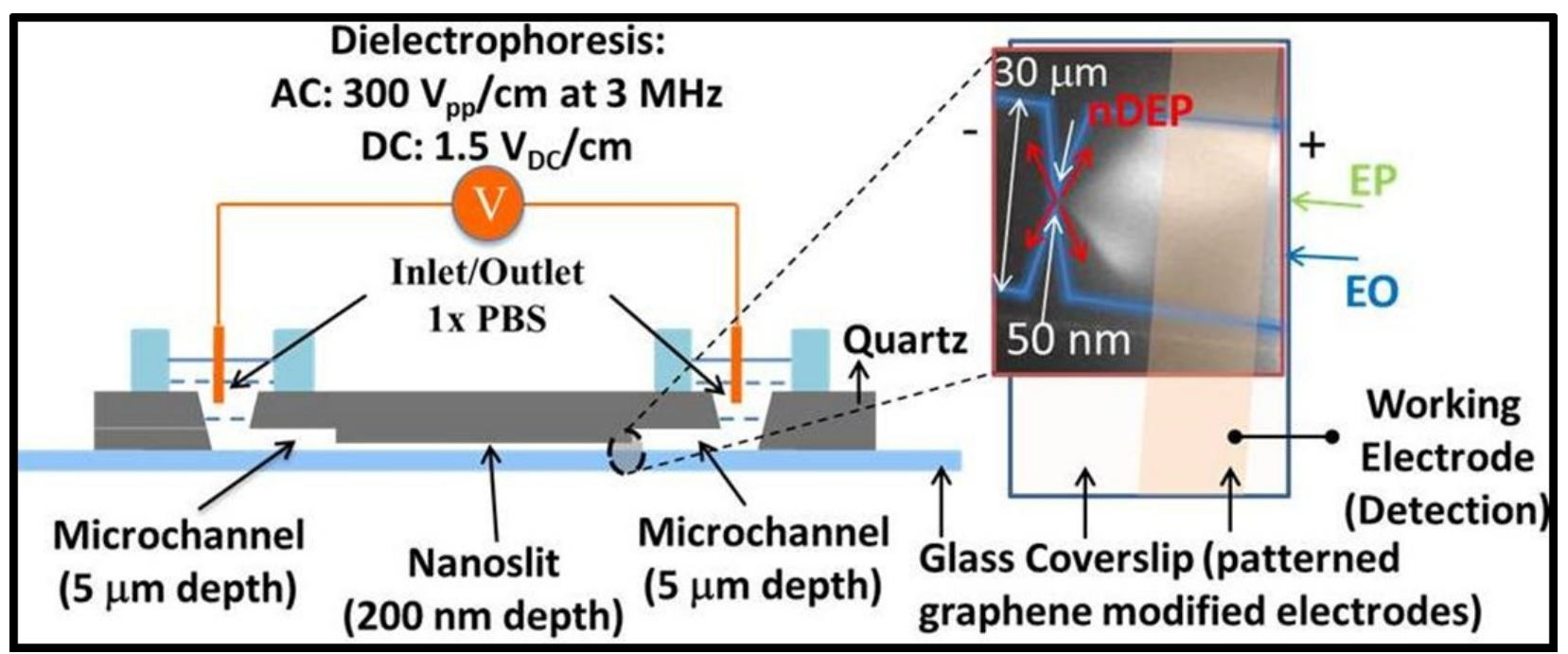

Figure 12: A; Cross section of integrated Nanoslit device. B; Graphene modified modified glassy carbon working electrode aligned to region of high electrokinetic preconcetration within nanoslit.[2]

electrode was functionalized with receptors. For the case of the NPY detection a Nafion (NAF) film was drop cast under a stereomicroscope onto the working electrode. For the case of PSA detection, PSA antibodies were thiolated[62] and bound to the AuNPs deposited on the working electrode. These anti-PSA capture probes enable analyte immobilization at the modified working electrode surface. The electrodes for electrochemistry were micropatterned inside a window of the PSQ-coated cover-slip for subsequent alignment to the constriction region of the nanoslit on the fused silica substrate, for bonding at room temperature. A room temperature bonding method is vital in order to preserve biofunctionality of deposited material, such as the immobilized anti-PSA capture probes in the case of the PSA detection scheme[61,63]. Prior to bonding, the length-edge of the working electrode was orthogonally aligned to be within $\sim 5 \mu \mathrm{m}$ from the constriction tip, to align the nDEP enrichment region of the biomarkers to the electrochemical detection electrodes. This device geometry ensures that the counter and reference electrodes are in the same nanochannel as the working electrode, thereby enabling sensitive current measurement and allowing all potential drops to occur at the surface of the working electrode, rather than in solution. [1, 2] 


\section{Nanofluidic device operation for ultra-fast NPY detection}

In order to facilitate integration of the electrodes for electrochemistry within the nanoslit, an optimization step is conducted to characterize NPYs preconcentration profile under frequency dependent n-DEP. This is done to determine optimal operational frequency and aligned working electrode placement within the preconcentration region. This is achieved by observing the preconcentration of fluorescently labeled NPY (Phoenix) under inverted microscopy (Zeiss, Z1) using an EMCCD (Hamamatsu) within the nanoslit device over a range of frequencies. The negative DEP behavior of NPY under an AC field of 300 $\mathrm{Vpp} / \mathrm{cm}$ at $3 \mathrm{MHz}$ frequency is driven by external Pt electrodes (at inlet and outlet) using a standard function generator (Agilent 3220) and voltage amplifier (FLC), with an additional DC field of $1.5 \mathrm{~V} / \mathrm{cm}$ to enhance transport of NPY towards the constriction. Following optimization, electrochemical analysis by Differential Pulse Voltammetry (DPV) is carried out (Solartron) using the patterned graphene-modified electrodes aligned to the region of highest preconcentration. For preconcentration of NPY, NPY 18-36 and OXA by electrochemical adsorptive accumulation, $-0.7 \mathrm{~V}$ is applied to the graphene-modified electrode (10 s to $450 \mathrm{~s}$ ), followed by DPV from +0.4 to $+1.0 \mathrm{~V}$, using a step potential of $5 \mathrm{mV}$ and modulation amplitude of $50 \mathrm{mV}$. For cyclic voltammetry (CV), the potential is scanned from $0.3 \mathrm{~V}$ to $+0.9 \mathrm{~V}$. Double potential step chronocoulometry is carried out with a pulse period of $5 \mathrm{~s}$ from $+0.45 \mathrm{~V}$ to $+0.95 \mathrm{~V} \mathrm{vs}$. $\mathrm{Ag} /$ $\mathrm{AgCl}$. Based on the amino acid sequences of NPY and NPY 18-36 and OXA, electrochemical oxidation of the tyrosine moiety involves five electrons for NPY, four electrons for NPY 18-36 and one electron for OXA:

$$
\begin{gathered}
N P Y: \text { Reduced }(\mathrm{Tyr}-\mathrm{R}-\mathrm{Tyr}-\mathrm{Tyr}-\mathrm{R}-\mathrm{Tyr}-\mathrm{R}-\mathrm{Tyr}) \stackrel{-5 e^{-}-5 H^{+}}{\longrightarrow} \text { NPY: Oxidized ... Eq. (1) } \\
N P Y 18-36: \text { Reduced ( } \mathrm{R}-\mathrm{Tyr}-\mathrm{Tyr}-\mathrm{R}-\mathrm{Tyr}-\mathrm{R}-\mathrm{Tyr}) \stackrel{-4 e^{-}-4 H^{+}}{\longrightarrow} \text { Oxidized ... Eq. (2) } \\
\text { OXA: Reduced (Tyr }-\mathrm{R}-\mathrm{Tyr}) \stackrel{-1 e^{--1 H^{+}}}{\longrightarrow} \text { OXA: Oxidized ... Eq. (3) }
\end{gathered}
$$




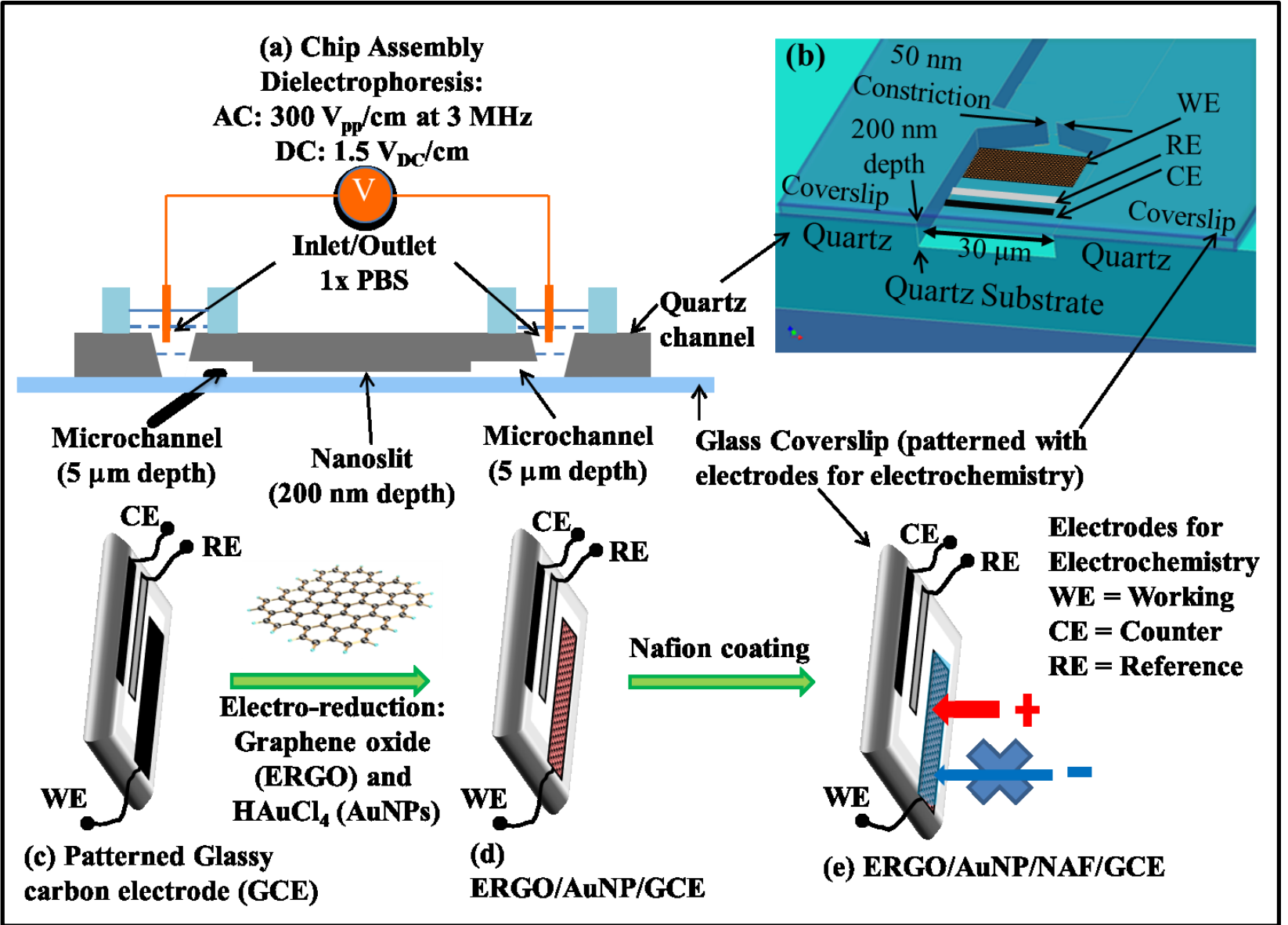

Figure 13: Cross-section (a) and top-view (b) of the chip assembly with microchannel reservoirs (5 $\mu m$ depth) and slit shaped nanochannels (200 $\mathrm{nm}$ depth) containing $\sim 50 \mathrm{~nm}$ lateral constrictions fabricated on a quartz substrate and bonded to a glass cover-slip with patterned electrochemical electrodes, including graphene-modified working electrode (fabricated as per: (c)(e)), $\mathrm{Ag} / \mathrm{AgCl}$ reference electrode and counter electrode that are aligned orthogonally to fit in the nanochannel in proximity of the lateral constriction. [2]

A supporting electrolyte of $0.1 \mathrm{M}$ phosphate buffer at $\mathrm{pH} 6.0$ leads to optimal electrochemical oxidation of NPY, as judged by the DPV peak current. The potential for electrochemical accumulation is optimized based on neuropeptide charge, determined from difference of the $\mathrm{pH}$ to $\mathrm{pKa}$ for each of the positive and negatively charged amino acids in the respective peptide[64]. At pH 6 in PBS electrolyte, the accumulation potential is $-0.7 \mathrm{~V}$ for NPY due to its net charge of ' +0.75 ', whereas a lower potential of $-0.5 \mathrm{~V}$ is sufficient for OXA due to its higher net charge of ' $+2.5^{\prime}$ ', and just $-0.3 \mathrm{~V}$ is sufficient for the accumulation of NPY 1836 due to its net charge of ' $+4.75^{\prime}$. The NAF cation exchange overlayer on the graphene-modified electrode enables the exchange of $\mathrm{H}+$ from NAF with the NPY+ (or OXA+) diffusing from the bulk electrolyte 


\section{Nanofluidic device operation for ultra-fast PSA detection}

As with the NPY detection scheme, the electrode placement is optimized to facilitate integration of DEP based enrichment with electrochemical detection within the nanoslit based on the PSA preconcentration profile under frequency dependent $n$-DEP. This is achieved by observation of the preconcentration of Dylight 488 labeled anti-PSA (Jackson ImmunoResearch Laboratories) and Dylight 594 labeled PSA (AbD Serotech) under inverted microscopy (Zeiss, Z1) using an EMCCD (Hamamatsu) within the nanoslit device over a range of frequencies. No fluorescently tags were used in subsequent electrochemical Integrated device operation. After optimization and integration of the Electrochemical detection electrodes within the nano-slit, three types of experiments were performed to compare the binding kinetics of PSA with immobilized antiPSA capture probes on the AuNPs on the ERGO modified GC electrode surfaces using electrochemical detection with: (i) $\sim 1 \mu \mathrm{L}$ scale droplets on the microfabricated cover-slip; (ii) nanoslit device $(\sim 0.1 \mathrm{~nL})$ integrated to the microfabricated cover-slip, with no nDEP enrichment; and (iii) nDEP enriched PSA within the nanoslit device integrated to the microfabricated cover-slip. For case (i), droplets of $\sim 1 \mu \mathrm{L}$ were incubated on SU8 patterned cover-slip with the microfabricated electrodes. For case (ii), a pulse-free flow system (neMESYS Syringe pump from Cetoni, Inc.) was used to fill microchannels, followed by application of 50 VDC at glassy carbon modified Pt

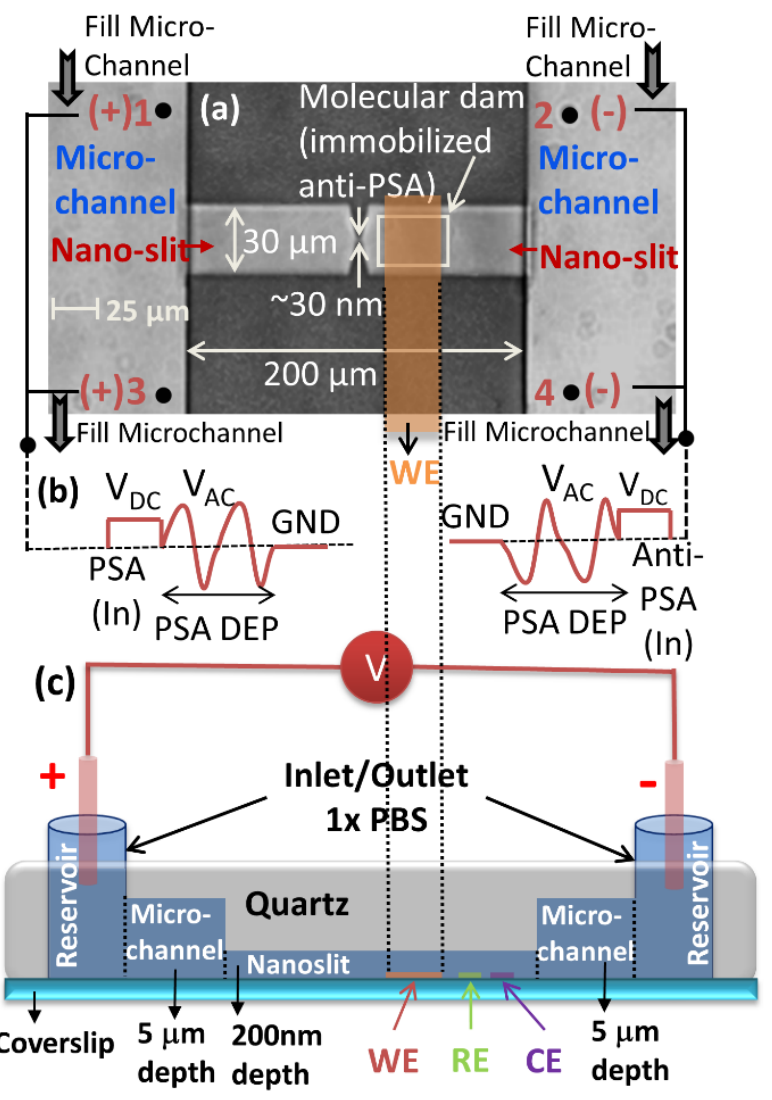

Figure 14: Nanoslit device: (a) Top-view of microchannels leading to nanoslit with lateral constrictions aligned to the molecular dam region with preconcentrated proteomic biomarkers, (b) electrical signal sequence at inlet/outlet ports 1 \& 3 (left) versus 2 \& 4 (right) after filling microchannels with biomarkers (left and right); and (c) cross-sectional view of nanoslit bonded to glass cover slip patterned with working $(W E)$, reference (RE) and counter electrodes (CE). The positive (left) and negative (right) indicates net DC offset during $n D E P \cdot[1]$ 
electrodes (Alfa Aesar) within each inlet/outlet reservoir for electrokinetic sample transport into the nanoslit. For case (iii), electrodes at the reservoirs were programmed to a sequence of electrical signals [42], as indicated in Figure 1b, with terminals (1) and (3) (left) versus (2) and (4) (right). The sequence involves a DC field of $50 \mathrm{~V} / \mathrm{cm}$ for $10 \mathrm{~s}$ to enable electrokinetic filling of PSA (concentration varied from 1 $\mathrm{pg} / \mathrm{mL}-5 \mathrm{ng} / \mathrm{mL}$ ) and NPP (100 mM) from the reservoirs, followed by the time period for which nDEP was conducted using $200 \mathrm{Vpp} / \mathrm{cm}$ at $6 \mathrm{MHz}$ with a DC offset of $1.5 \mathrm{~V} / \mathrm{cm}$, and finally, a DC field $(50 \mathrm{~V} / \mathrm{cm}$ for $5 \mathrm{~s})$ to enable electrokinetic filling ALP-tagged secondary anti-PSA and BSA from the reservoirs into the nanoslit. Square-wave voltammetric (SWV) scans of $\alpha$-NP were performed in the pH 6.5 PBS media by scanning from 0.1 to $0.4 \mathrm{VDC}$, using a $100 \mathrm{~Hz}$ frequency; $4 \mathrm{mV}$ step potential; and $25 \mathrm{mV} \mathrm{AC}$ amplitude. The time on the kinetic plots is the time for PSA DEP (under AC field) plus the short time for transport of secondary antibody to the nanoslit ( $\sim \mathrm{s}$ of VDC). Solely for the case of analysis within the $1 \mu \mathrm{L}$ droplet, a short accumulation time (10 s) at a deposition potential of $-0.1 \mathrm{~V}$ was used to enhance adsorption. Standard deviations $(95 \%$ confidence levels) were based on three different electrochemical measurements at each time point for each of (a) Immobilization the PSA concentration levels reported herein.

\section{Modification of secondary PSA} antibody for sandwich assay

The sandwich assay for electrochemical detection was performed using Alkaline phosphatase (ALP) tagged to the secondary PSA antibody by glutaraldehyde coupling [65], for enabling the selective catalysis of $\alpha$-naphthyl phosphate (NPP) to the electroactive $\alpha$ naphthol (NP) product [62], as per the detection scheme in Figure 15. For ALP tagging of antiPSA, an appropriate amount of ALP solution
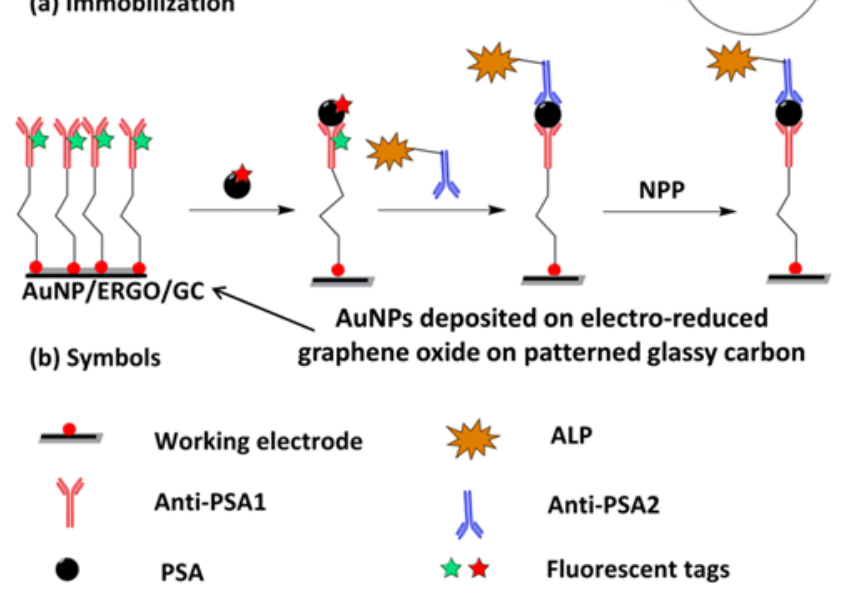

(c) Electrochemical Detection

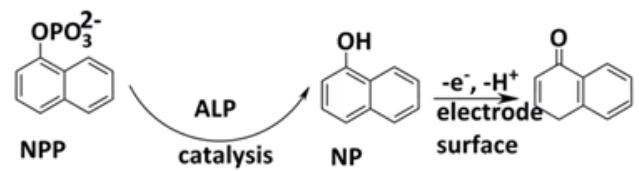

Figure 15: : Detection scheme for PSA: (a) Immobilization of antiPSA capture probes on AUNP modified graphene/glassy carbon surfaces for a PSA sandwich assay using ALP-tagged secondary antibodies; (b) Symbols; (c) Electrochemical detection method. Note: Fluorescent tags applied only for optimizing the device and Efield for nDEP enrichment. [1] 
was transferred to an Eppendorf tube and mixed with anti-PSA in deionized water. Next, 1\% glutaraldehyde in $0.1 \mathrm{M}$ phosphate buffer $(\mathrm{pH} 7.4)$ was added to the solution. The resulting mixture was incubated with shaking $(120 \mathrm{rpm})$ at room temperature for $10 \mathrm{~min}$, and then incubated for 4 hours in the dark. Finally, $1 \mathrm{M}$ monoethanolamine solution was added to the mixture, which was subsequently incubated with shaking (100 rpm) for $2 \mathrm{~h}$ at room temperature. The mixture was dialyzed against PBS solution at $4{ }^{\circ} \mathrm{C}$ overnight. The dialysis product, PSA antibody-ALP conjugate, was then transferred to an Eppendorf tube and mixed with an equal volume of glycerin and $1 \% \mathrm{BSA}$, for storage at $0{ }^{\circ} \mathrm{C}$, prior to the experiments. 


\section{Chapter 3: Results and Discussion}

\section{Aim One Results: Deformability based separation of islets}

\section{Limitations on islet purity after density gradient separation}

The limitations on \% purity of the islet samples generated by the density gradient (DG) method are explored here based on 22 islet isolation procedures using this method on human donor research organs. Based on estimated purity levels, the packed tissue volume of islets and acinar aggregates within each of the 12 fractions collected from the COBE system for DG separation is plotted in Fig. 16a. It is noteworthy that aggregates of acinar tissue are predominantly trapped within the higher density media, thereby appearing primarily in the \#9-12 COBE fractions, while the islets are broadly distributed over the central centrifuged fractions: \#3-8 from COBE system. As is typically done prior to incubation, the islet containing fractions were combined into four bins, based on the sample purity levels (i.e. \% islets): high purity $(>80 \%)$, medium purity $(80 \%-50 \%)$, low purity $(50 \%-30 \%)$ and waste $(<30 \%)$ samples. Within these four bins, Fig. 16b shows the distribution of islet volume (estimated from the purity and packed tissue volume of the combined fraction), while Fig. 16c shows the total packed tissue volume after each isolation step. Based on this, it is apparent that while the islet volume remains relatively constant across the low, medium and high purity sample bins (each representing $28 \%$ of the overall islet yield from donor organs), the total packed tissue volume within these bins drops with increasing islet purity levels, thereby indicating that the transplant plug cannot be generated solely from a single sample bin, due to the insufficient islet numbers within the high purity sample bin. Currently, islet transplant samples must meet the following criteria: (i) the total number of islets must be greater than 5,000 for every kg of the recipients body weight; (ii) viable islets must form $30 \%$ of the transplant plug, with a viable islet defined as those with at least $70 \%$ of component cells lacking the propidium iodide (PI) stain; and (iii) the total transplant 


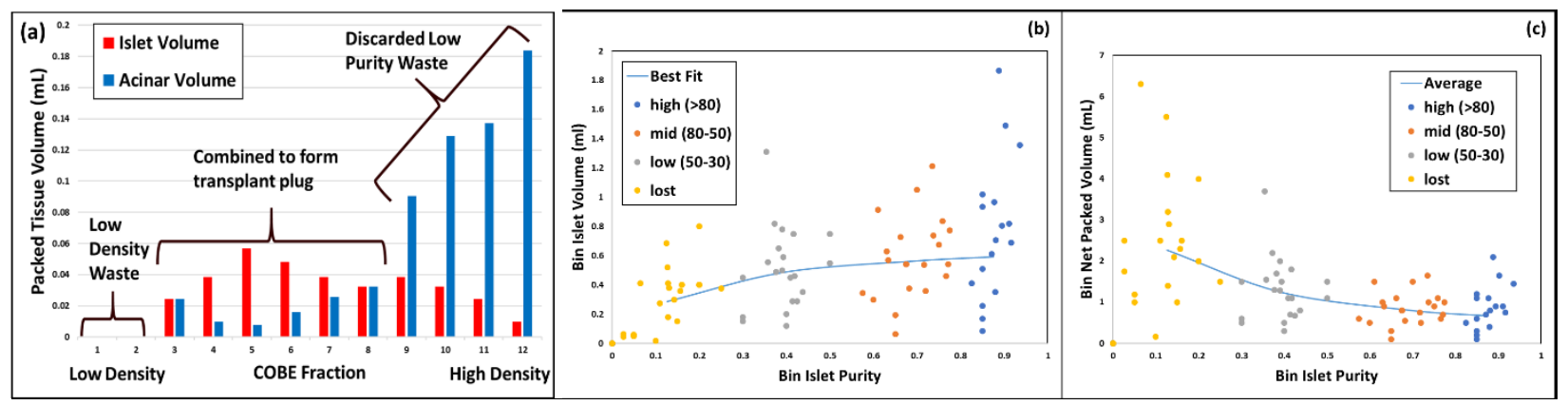

Figure 16: (a) Packed tissue volume ( $\mathrm{mL}$ ) of islets versus acinar within each of the centrifuged fractions from COBE system; (b) packed tissue volume for islets and (c) Total packed tissue volume within the bins characterized as: high purity (blue: > 80\%)), medium purity (orange: 50-80\%)), low purity (grey: 30-50\%) and waste samples (yellow: <30\% purity).

must be below $10 \mathrm{~mL}$ of packed tissue. Since islet yield from the two starting donor organs is relatively evenly distributed across the low, medium and high purity sample bins, the bins must be combined to form a transplant plug of less than $10 \mathrm{~mL}$ pact tissue volume. Based on this, from sixteen donor research organs used in the current investigation, we combined the isolated islet samples from high, medium, and low purity bins to form eight potential transplant plugs. The average purity of the transplant plug produced by this method was found to be around $60 \%$, with none of the transplants exceeding $72 \%$ and the average packed tissue volume of the plug was around $5.55 \mathrm{~mL}$, with none exceeding $8.5 \mathrm{~mL}$. Due to the inability of the sample bins generated by the density gradient separation method to reach higher purity levels, we infer that there is a significant level of density overlap between the pancreatic islets and a significant quantity of the acinar tissue. Hence, while the DG separation method is able to remove a majority of the acinar tissue aggregates, the generated transplant plug contains $\sim 40 \%$ acinar tissue aggregates, due to large acinar levels within the pre-purification slurry[66] and the wide distribution of islets across the collected sample bins, thereby leading to a transplant plug with relatively low purity. While this plug meets the current transplant requirements, it is likely that the high volume of acinar tissue within the transplant plug exacerbates the autoimmune response, resulting in an increased likelihood of transplant rejection[66, 67]. Furthermore, the waste sample bin (i.e. those with $<30 \%$ purity) of high volume that is usually discarded causes a $15 \%$ loss in the overall islets from the donor organ, representing an $85 \%$ collection efficiency. Finally, within about $20 \%$ of the DG separation cases investigated for this study, the method failed to produce a high purity sample bin ( $>80 \%$ islets), suggesting a great degree of variability in the isolated fractions. In the subsequent sections, we investigate the ability of the 
microfluidic deformability-based separation to enhance the islet purity from the transplant plug and from the waste sample bin of the DG method, since the former can alleviate immune responses while the latter can lower islet wastage from the donor organs.

The limitations on \% purity of the islet samples generated by the density gradient (DG) method are explored here based on 22 islet isolation procedures using this method on human donor research organs. Based on estimated purity levels, the packed tissue volume of islets and acinar aggregates within each of the 12 fractions collected from the COBE system for DG separation is plotted in Fig. 16a. It is noteworthy that aggregates of acinar tissue are predominantly trapped within the higher density media, thereby appearing primarily in the \#9-12 COBE fractions, while the islets are broadly distributed over the central centrifuged fractions: \#3-8 from COBE system. As is typically done prior to incubation, the islet containing fractions were combined into four bins, based on the sample purity levels (i.e. \% islets): high purity (>80\%), medium purity $(80 \%-50 \%)$, low purity $(50 \%-30 \%)$ and waste $(<30 \%)$ samples. Within these four bins, Fig. 16b shows the distribution of islet volume (estimated from the purity and packed tissue volume of the combined fraction), while Fig. 16c shows the total packed tissue volume after each isolation step. Based on this, it is apparent that while the islet volume remains relatively constant across the low, medium and high purity sample bins (each representing $\sim 28 \%$ of the overall islet yield from donor organs), the total packed tissue volume within these bins drops with increasing islet purity levels, thereby indicating that the transplant plug cannot be generated solely from a single sample bin, due to the insufficient islet numbers within the high purity sample bin. Currently, islet transplant samples must meet the following criteria: (i) the total number of islets must be greater than 5,000 for every kg of the recipients body weight; (ii) viable islets must form $30 \%$ of the transplant plug, with a viable islet defined as those with at least $70 \%$ of component cells lacking the propidium iodide (PI) stain; and (iii) the total transplant must be below $10 \mathrm{~mL}$ of packed tissue. Since islet yield from the two starting donor organs is relatively evenly distributed across the low, medium and high purity sample bins, the bins must be combined to form a transplant plug of less than $10 \mathrm{~mL}$ pact tissue volume. Based on this, from sixteen donor research organs used in the current investigation, we combined the isolated 
islet samples from high, medium, and low purity bins to form eight potential transplant plugs. The average purity of the transplant plug produced by this method was found to be around $60 \%$, with none of the transplants exceeding $72 \%$ and the average packed tissue volume of the plug was around 5.55 $\mathrm{mL}$, with none exceeding $8.5 \mathrm{~mL}$. Due to the inability of the sample bins generated by the density gradient separation method to reach higher purity levels, we infer that there is a significant level of density overlap between the pancreatic islets and a significant quantity of the acinar tissue. Hence, while the DG separation method is able to remove a majority of the acinar tissue aggregates, the generated transplant plug contains $\sim 40 \%$ acinar tissue aggregates, due to large acinar levels within the pre-purification slurry[66] and the wide distribution of islets across the collected sample bins, thereby leading to a transplant plug with relatively low purity. While this plug meets the current transplant requirements, it is likely that the high volume of acinar tissue within the transplant plug exacerbates the autoimmune response, resulting in an increased likelihood of transplant rejection[66],[67]. Furthermore, the waste sample bin (i.e. those with $<30 \%$ purity) of high volume that is usually discarded causes a $15 \%$ loss in the overall islets from the donor organ, representing an $85 \%$ collection efficiency. Finally, within about $20 \%$ of the DG separation cases investigated for this study, the method failed to produce a high purity sample bin ( $>80 \%$ islets), suggesting a great degree of variability in the isolated fractions. In the subsequent sections, we investigate the ability of the microfluidic deformability-based separation to enhance the islet purity from the transplant plug and from the waste sample bin of the DG method, since the former can alleviate immune responses while the latter can lower islet wastage from the donor organs.

\section{Size and deformability overlaps for mouse islets versus acinar particles}

In order to establish the basis for utilizing deformability as the basis for separation of islets from acinar tissue aggregates, we compare the overlaps in the distribution of size and deformability for islets versus acinar aggregates. For this purpose, pure samples of islets and acinar aggregates isolated from mouse 


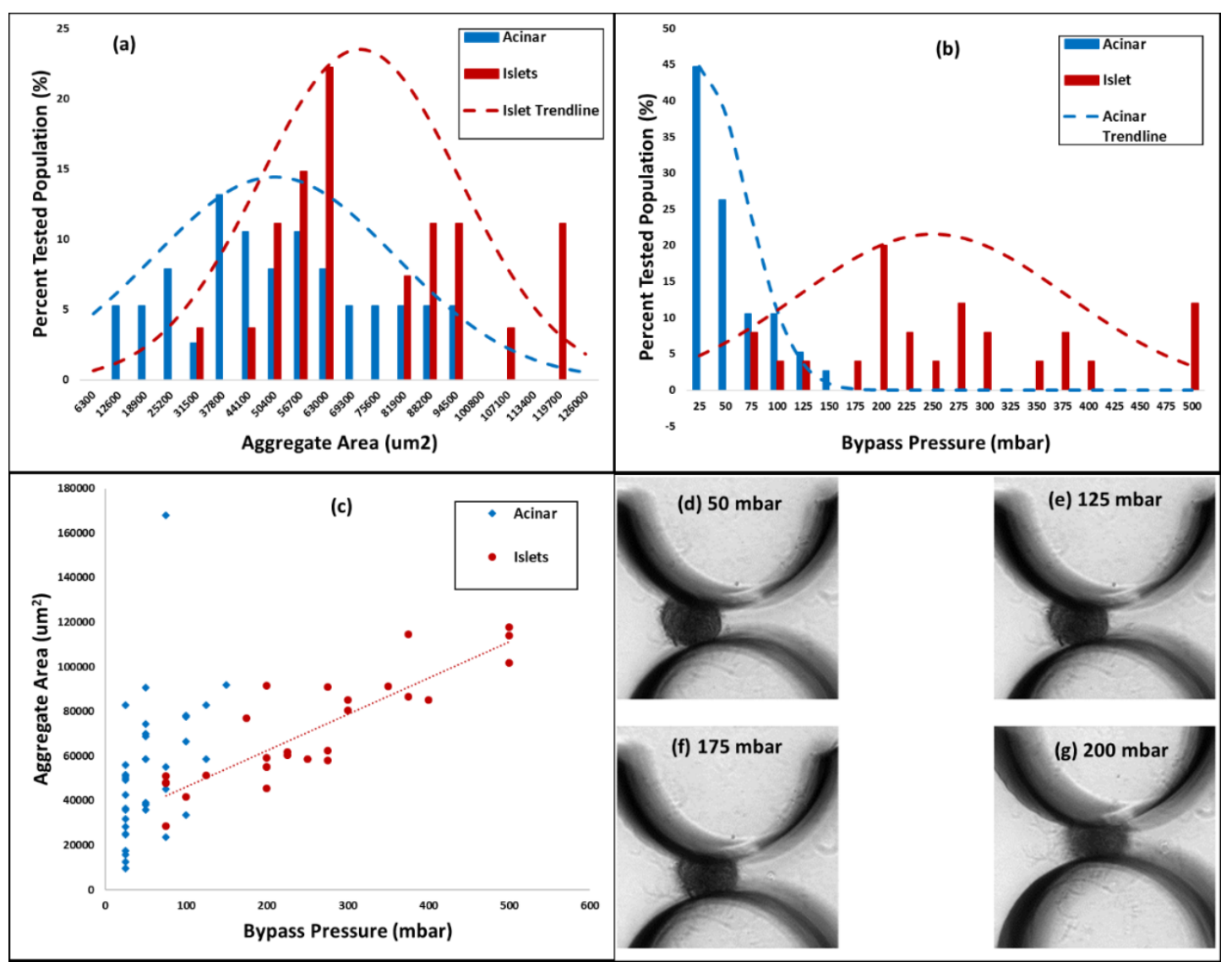

Figure 17: (a) Size overlaps of acinar versus islet particles are shown as a histogram of the respective particle area; (b) Deformability overlaps of acinar versus islet particles are shown as a histogram of the threshold bypass pressure (mbar) for passage of the respective particles; (c) Scatter plot of the area of the respective aggregate (islet or acinar) to their threshold bypass pressure shows that a clear linear relationship is apparent only for the islets; (d) Example threshold bypass pressure measurement for a typical islet at 200 mbar pressure through an $80 \mu \mathrm{m}$ constriction.

pancreas were measured after 24 hours of enzymatic digestion, for sizes based on pixel area and deformability based on threshold bypass pressure for passage through $80 \mu \mathrm{m}$ constrictions (using the device in Fig. 1). As per Fig. 17a, while the average sizes of the islets are significantly larger than those of acinar tissue populations, there is a considerable degree of overlap, which is somewhat consistent with their poor separation under the DG method, likely due to density overlaps. On the other hand, Fig. 17b shows a relatively small degree of deformability overlap for islets versus acinar tissue aggregates, since just $9 \%$ of the islet population and $19 \%$ of the acinar population have deformabilities that lie within the overlap region. These overlaps become insignificant at applied pressure differentials beyond 150 mbar. Images of the passage of islets through $80 \mu \mathrm{m}$ constrictions at a threshold bypass pressure of 200 mbar are shown in Fig. 17d-17g, to illustrate the overall experiment. The plot in Fig. 17c shows 


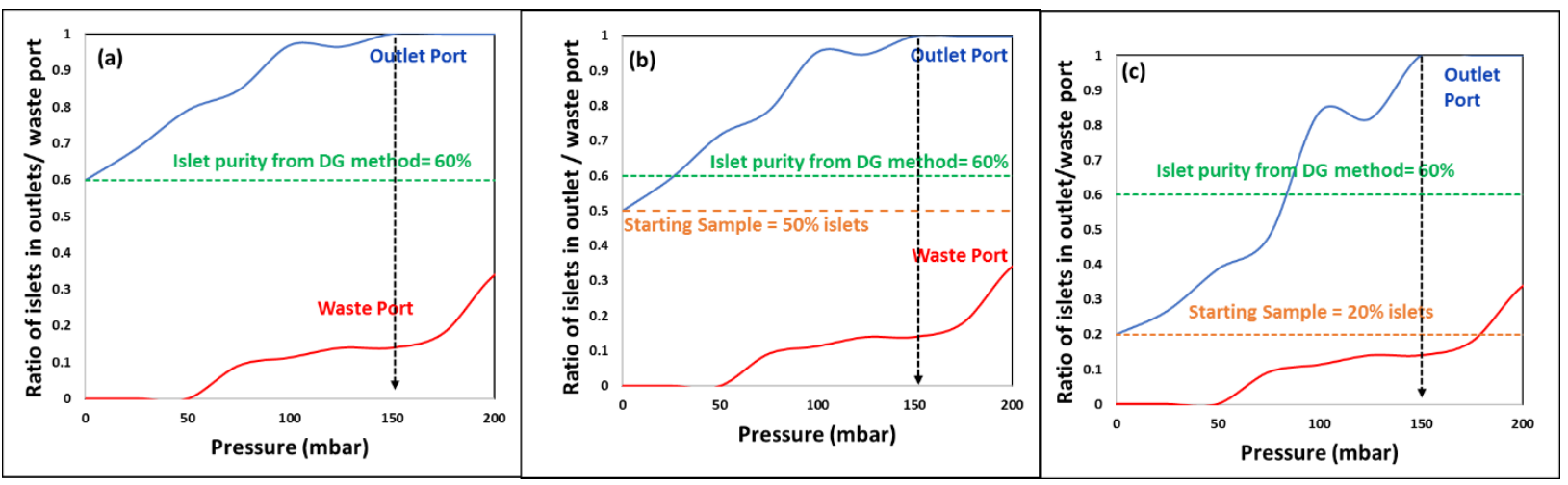

Figure 18: Computed ratio of islet levels obtained in the outlet (separation purity) and waste ports (sample loss) within a separation device obtained with pressure differentials up to 200 mbar for starting samples of: (a) 60\% islets (such as after DG separation); (b) 50\% islets (intermediate islet levels); (c) 20\% islets (discarded sample from DG method).

the data in terms of aggregate area versus threshold bypass, which suggests that while bypass pressure

of islets rises linearly with their area, the same is not apparent with acinar populations likely due to a more heterogeneous deformability distribution. The deformability overlap of islet versus acinar populations in Fig. 17b is used to quantify the possible separation purity and collection efficiency numbers within a separation device, by computing the anticipated islet purity and sample loss using applied pressure levels up to 200 mbar, for various starting samples: 60\% purity within Fig. 18a (as obtained after the DG separation method); 50\% purity within Fig. 18b (an intermediate level of islet purity) and 15\% purity within Fig. 18c (as obtained from the discarded residue of the DG separation method). These plots suggest a pressure differential of 50 mbar can enrich islets with near-zero sample loss from $60 \%$ to $80 \%$ (Fig. 18a), from $50 \%$ to $70 \%$ (Fig. 18b), and from $15 \%$ to $30 \%$ levels (Fig. 18c). On the other hand, if sample losses of $10-15 \%$ can be tolerated, an islet sample with $95 \%$ purity can be obtained from a starting sample after the DG separation method (60\% purity) using a 100 mbar pressure differential and islet samples with near $100 \%$ purity can be obtained from all three starting samples considered herein (60\%, 50\% and $15 \%)$ using a 150 mbar pressure differential.

\section{Microfluidic separation of islet versus acinar particles}

The microfluidic device of Fig. $\mathbf{4 d}$ was applied as per the separation methodology described in Section 2.2 to quantify the degree of islet enrichment for two different starting islet samples: $18 \%$ and 


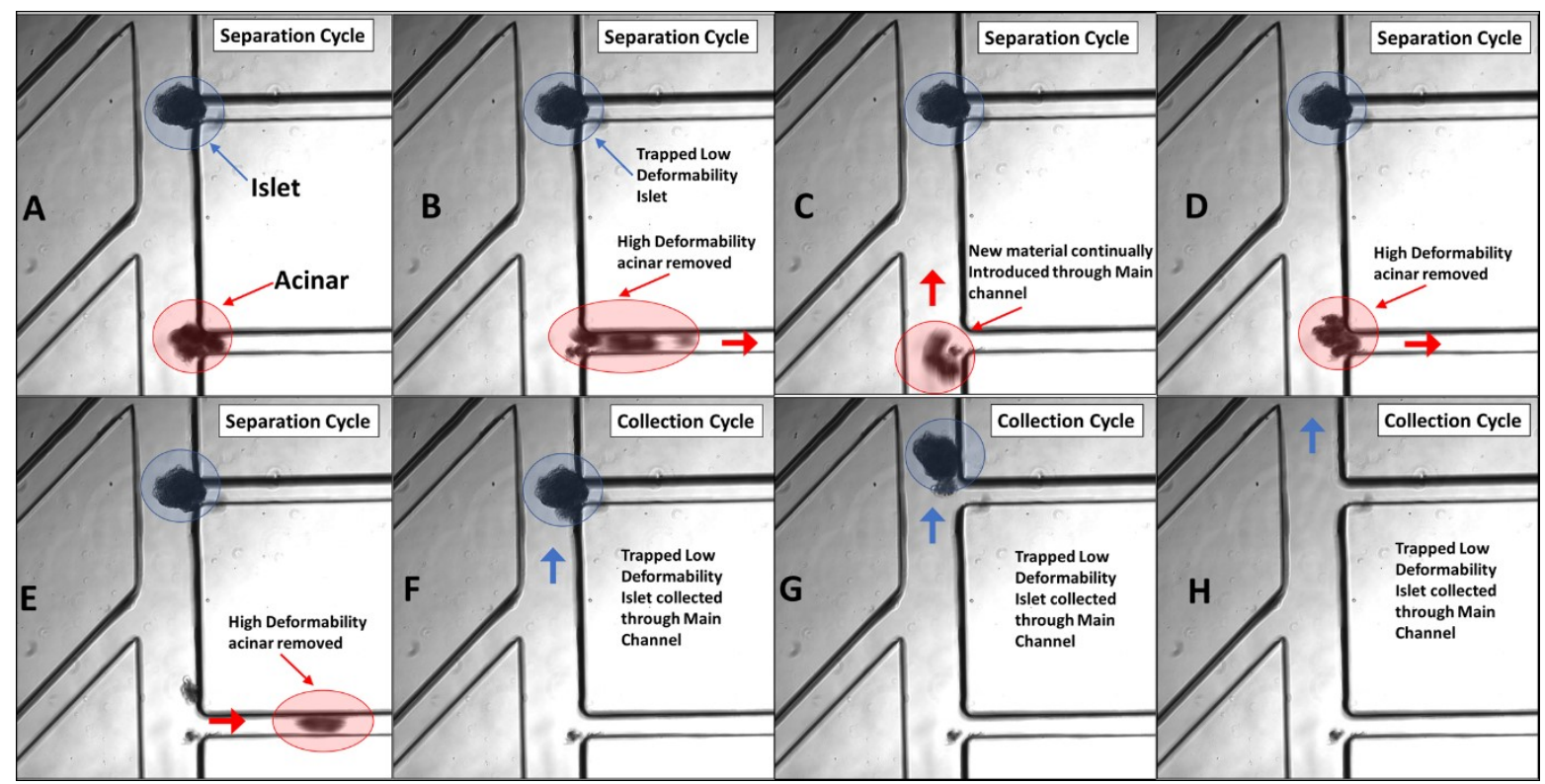

Figure 19: The microfluidic device of Fig. 2 is applied under the separation mode at an applied pressure differential of 150 mbar to remove acinar populations (indicated as red circles) from the bifurcating points towards the waste port (A-E), while the collection mode is used to collect the trapped islets at the bifurcating points (indicated as blue circles) towards the main outlet port $(F-H)$.

$56 \%$. For the islet sample with $18 \%$ purity, the separation mode was operated for 79 seconds followed by operation in the collection mode for 20 seconds, while for sample with $56 \%$ purity the separation mode was operated for 21 seconds followed by operation in the collection mode for 20 seconds. As per the images in Fig. 19 for sample entry and placement at bifurcating points through the array of focusing flows, islet particles are trapped at the bifurcating points (Fig. 19a \&19c) during the separation mode, while acinar particles are removed Undamaged Islets

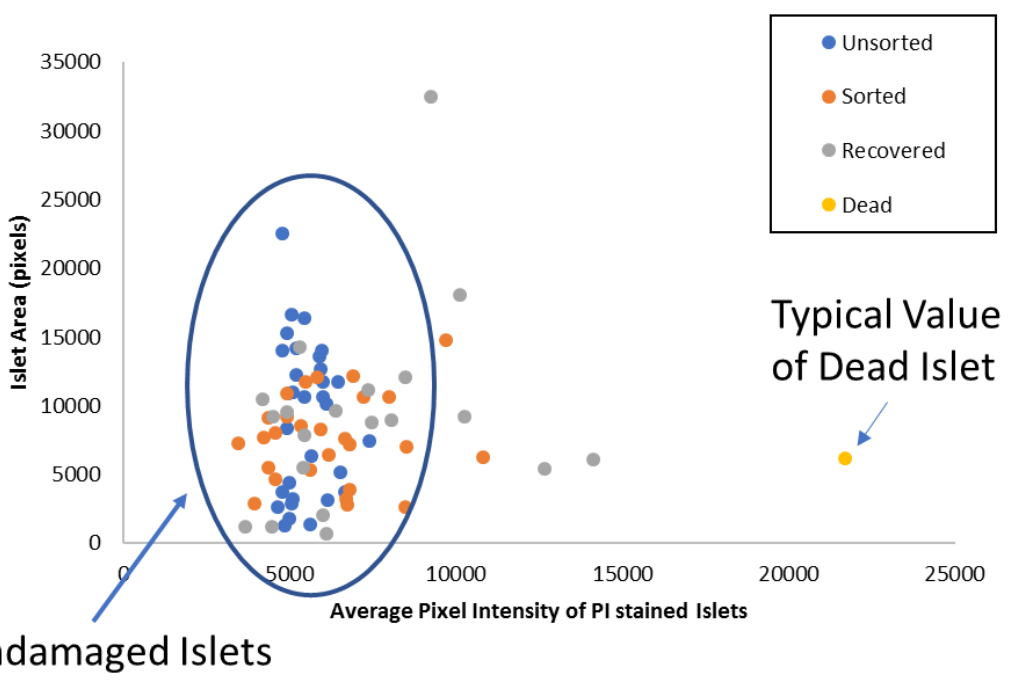

Figure 20:The average pixel intensity versus size of PI stained islets without being sorted in the microfluidic device (unsorted blue), while being sorted (being sorted orange), and after by deformation from the being recovered from the microfluidic device (recovered grey) with a reference point (yellow) for the typical florescent value of a non-viable islet.

bifurcating point through to the waste port (Fig. 19b \& 19d). It is clear that the trapped islets at a given bifurcating point do not hinder the flow of acinar particles to the successive bifurcating points, so that the 
trapped islets (Fig. 19e) can then be isolated under the collection mode. Table $\mathbf{2}$ shows the key figures of merit on measured islet purity and sample loss for two starting samples ( $18 \%$ and $56 \%$ purity) in comparison to the respective computed numbers, based on the deformability overlap in Fig. 17b. The relatively good correlation between the measured and computed parameters confirms the significant role of deformability-based differences within the observed separation. These results demonstrate that the microfluidic deformability-based separation methodology can enable enrichment of islets from samples with a wide distribution of starting islet purities, including samples obtained after DG separation ( $\sim 60 \%$ purity) and the rather dilute samples discarded by the DG separation method ( $<20 \%$ purity). The isolated islets by this microfluidic method retain their viability, as apparent from Fig $\mathbf{2 0}$ which plots the results of a Pi viability stain. As islet viability is compromised, more of the membranes of the cells that comprise the islet become highly permeable, increasing the measured average florescent pixel intensity of a PI stained islet. Fig $\mathbf{2 0}$ plots the measured average pixel intensity of three groups of islets, pristine islets which have not been subjected to the microfluidic sorting(unsorted), Islets within the microfluidic environment (being sorted), and Islets which have been recovered from the microfluidic device (recovered). As this plot demonstrates though the islets due exhibit some damage from the sorting process, particularly from the recovery process, their average pixel intensities are within acceptable limits.

Viability of islets isolated by microfluidic device

\begin{tabular}{|l|l|l|l|l|}
\hline Figure of merit (area based measurements) & Measured & Predicted & Measured & Predicted \\
\hline Starting islet level & $18 \%$ & $18 \%$ & $56 \%$ & $56 \%$ \\
Islet purity after separation & $62 \%$ & $82 \%$ & $88 \%$ & $96 \%$ \\
Acinar removed & $87 \%$ & $95 \%$ & $83 \%$ & $98 \%$ \\
Loss in islets (waste port) & $5 \%$ & $0 \%$ & $0 \%$ & $0 \%$ \\
Volume reduction (collected sample) & $62 \%$ & $78 \%$ & $37 \%$ & $42 \%$ \\
\hline
\end{tabular}

Table 2 Comparison of the transplant samples after microfluidic separation of islets from acinar tissue populations for two starting islet levels, as obtained by measurements versus those predicted based on computation from the observed deformability overlaps of the respective populations.

Islets obtained after sorting by the described microfluidic techniques were compared to the pristine islets (so-called unsorted) that were not subject to microfluidic device passage by studying their ability to vascularize using a mouse cornea micropocket assay conducted by a collaborator, Molly Kelly-Goss. As seen in Figure 21a, the mouse cornea is a clear, avascular tissue available for exogenous islet 
transplantation. By placing an islet in the corneal epithelial layer, close to the limbus, we are able to observe the viability and vascularity of the islets (Figure $\mathbf{2 1} \mathbf{b}$-d,f-h). Figure 8 e shows a 27 -gauge needle being used to place islets into the cornea. After transplantation, both sorted (Figure 21b-d) and unsorted (Figure 21f-h) islets remain viable in the tissue and their vasculature is able to incorporate with the capillaries surrounding the mouse cornea. Figures $\mathbf{2 1 c}$ and $\mathbf{2 1 f}$ show sorted and unsorted pancreatic islets seven days after being placed into the cornea, respectively. The respective islets are viable and incorporated into the local vasculature, as shown by capillaries labeled for an endothelial cell marker, CD31, in Figures $\mathbf{2 1 c}$ and $\mathbf{2 1 g}$. The depth of the vasculature in the tissue Figures $\mathbf{2 1 d}$ and $\mathbf{2 1} \mathbf{h}$ is indicated colormetrically, where cooler-colored (blue-cyan) blood vessels are further away from the warmercolored (red-orange) vessels. Based on this, we can see vessels that travel from the limbus (in red) into the islet (in blue), indicating that the islets have been successfully vascularized and remain viable in the tissue.
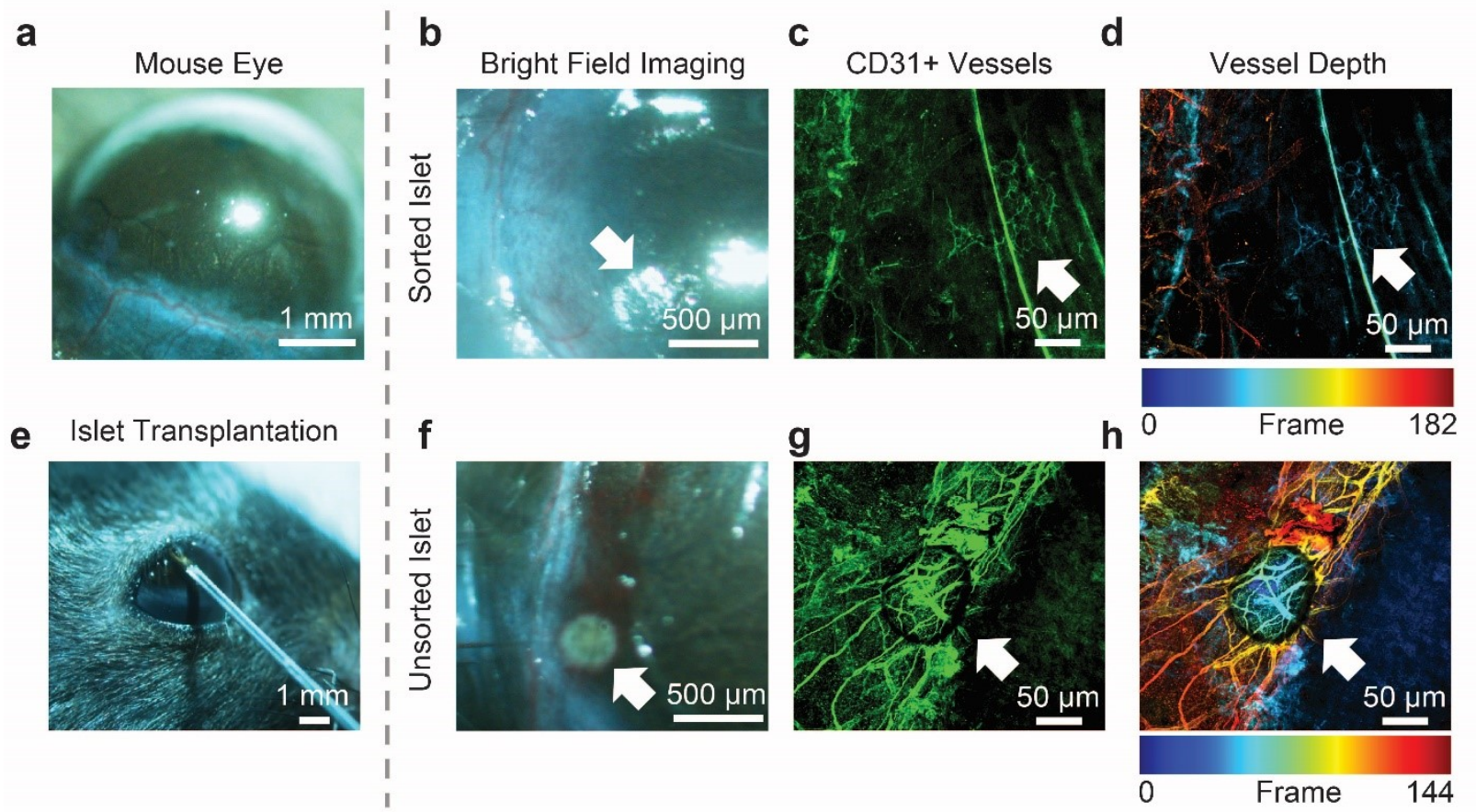

Figure 21: Islet angiogenic capacity is retained after sorting. (a) Sorted and unsorted pancreatic islets were placed into the mouse cornea using the corneal micropocket assay, $(b, f)$ where they remained viable for at least seven days. $(c-d, g-h)$ Both sorted and unsorted islets incorporated their vasculature into the nearby capillary network (white arrow indicates sorted pancreatic islet). (c, $g$ ) Capillaries labeled with the endothelial cell marker $C D 31(d, h)$ were observed going from the limbus vessels (red-orange) into the pancreatic islet (blue-cyan) in the corneal epithelium seven days after transplantation. 


\section{Size and deformability overlap human derived islets versus acinar particles}

In order to substantiate the results of our deformability based separation method towards clinically relevant human tissue we again compared overlaps in the distribution of size and deformability for islets versus acinar aggregates derived from human donor organs. We further compared the results with human donor tissue to those of mouse derived tissue. For this purpose, high purity samples of islets and acinar aggregates isolated from a human donor pancreas were measured after enzymatic digestion and density gradient separation, for sizes based on pixel area and deformability based on threshold bypass pressure for passage through $80 \mu \mathrm{m}$ constrictions (using the device in Fig. 1). Due to the rarity of available

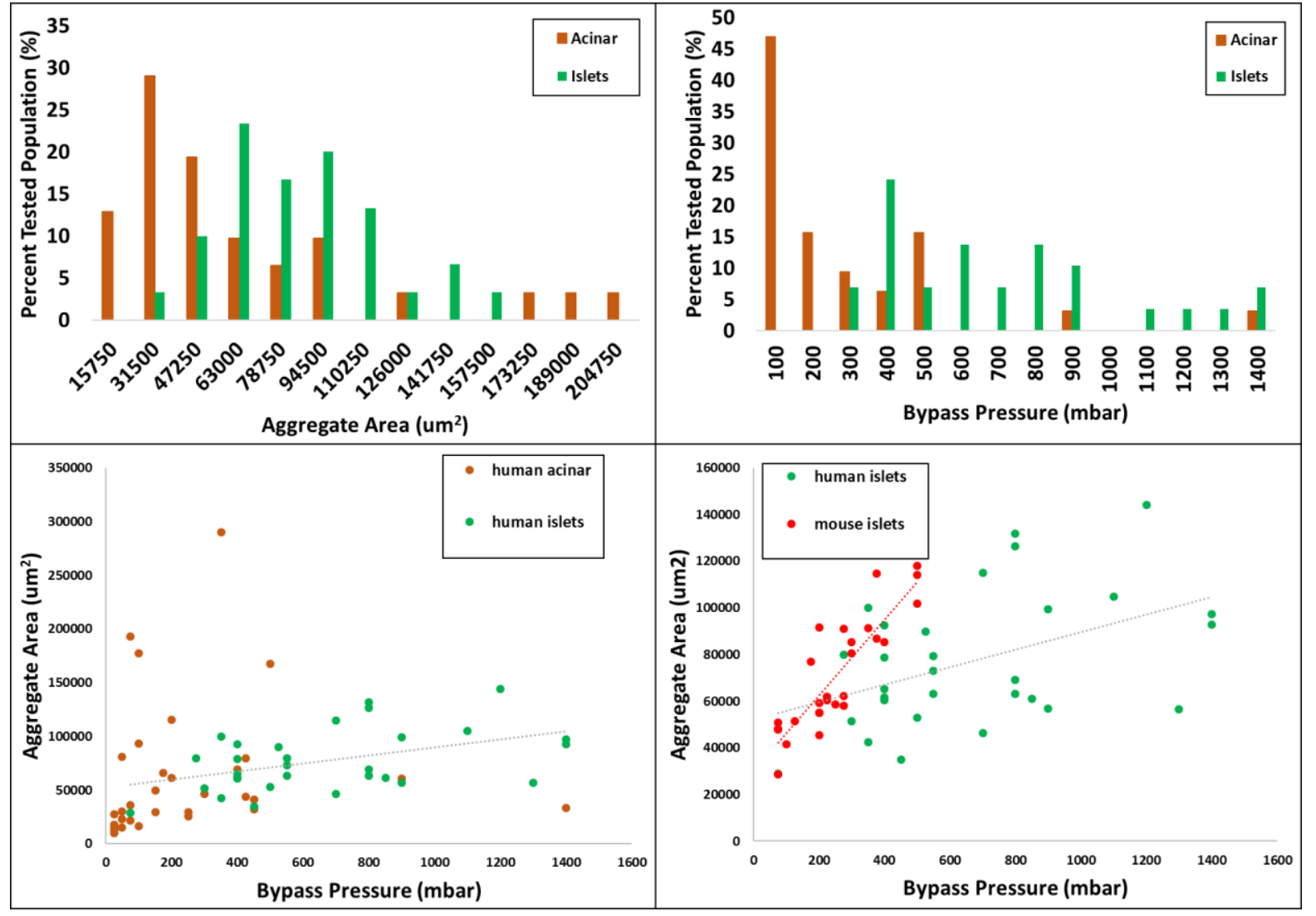

Figure 22 (a) Size overlaps of acinar versus islet particles are shown as a histogram of the respective particle area; (b) Deformability overlaps of acinar versus islet particles are shown as a histogram of the threshold bypass pressure (mbar) for passage of the respective particles; (c) Scatter plot of the area of the respective aggregate (islet or acinar) to their threshold bypass pressure shows that a clear linear relationship is apparent only for the islets; (d) Scatter plot of the area of the respective islets (human versus mouse) to their threshold bypass pressure shows that different linear relationships are apparent for the human versus mouse islets;

human transplant tissue measurements were taken over several days as opposed to only 25 hours after digestion as with the mouse tissue. As per Fig22., while the average sizes of the islets are generally larger 
than those of acinar tissue populations, there is a considerable degree of overlap, which is somewhat consistent with their poor separation under the DG method. When the human tissue size range is compared with that of the mouse derived tissue, as per Fig22., the size range of the human tissue is shown to be significantly larger. Furthermore, the overlap of the size range of human acinar versus islet tissue aggregates is shown to be more significant for the human donor tissue. On the other hand, Fig. 22b shows a relatively small degree of overlap for islets versus acinar tissue aggregates, with $62.5 \%$ of acinar aggregates completed outside of the overlap regions. This is higher overlap than with the mouse tissue, which shows just $9 \%$ of the islet population and $19 \%$ of the acinar population have deformability that lie within the overlap region. The plot in Fig. 22c shows the data in terms of aggregate area versus threshold bypass. As with the mouse tissue, these results suggest that while bypass pressure of islets rises linearly with their area, the same is not apparent with acinar populations, suggesting a more heterogeneous deformability distribution. The plot in Fig. $22 \mathrm{~d}$ further compares human to mouse islet tissue in these terms. This plot demonstrates that not only do human islets tend to have a wider size range and bypass the constriction at higher pressures, but that the linear rise in bypass pressure with size has a shallower slope for the human islets. This suggests that the deformability of the human islet tissue is lower than that derived from mice.

The deformability overlap of islet versus acinar populations in Fig. $\mathbf{2 2} \mathbf{b}$ is used to quantify the possible separation purity and collection efficiency numbers within a separation device, by computing the anticipated islet purity and sample loss using applied pressure levels up to $600 \mathrm{mbar}$, for various starting samples: 60\% purity within Fig. 23a (as obtained after the DG separation method); 50\% purity within Fig. 23b (an intermediate level of islet purity) and 20\% purity within Fig. 23c (as obtained from the discarded residue of the DG separation method). These plots suggest an applied pressure of 250 mbar can enrich islets with near-zero sample loss from $60 \%$ to $77 \%$ (Fig. 23a), from $50 \%$ to $69 \%$ (Fig. 23b), and from $20 \%$ to $35 \%$ levels (Fig. 23c). On the other hand, with a sample losses of $31 \%$, an islet sample with $96 \%$ purity 
can be obtained from a starting sample after the DG separation method (60\% purity) using a 500mbar pressure differential and islet samples.
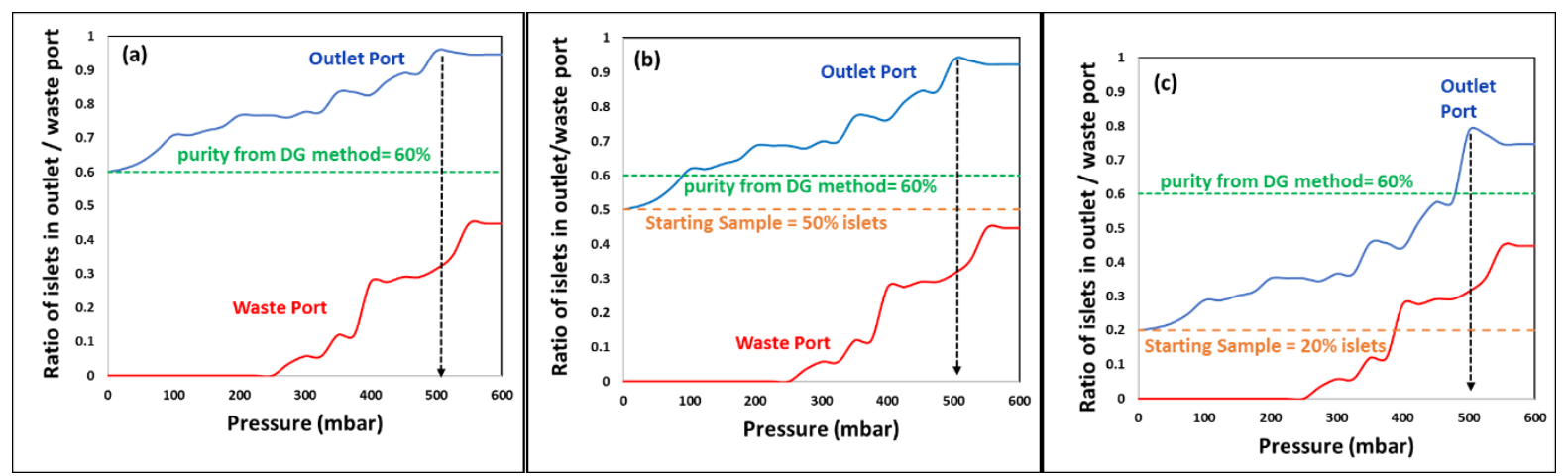

Figure 23: Computed ratio of islet levels obtained in the outlet (separation purity) and waste ports (sample loss) within a separation device obtained with pressure differentials up to 200 mbar for starting samples of: (a) 60\% islets (such as after DG separation); (b) 50\% islets (intermediate islet levels); (c) 20\% islets (discarded sample from DG method)

\section{Aim Two Results: Electrokinetic preconcentration and electrochemical}

\section{detection within a nanoslit platform}

\section{Preconcentration and electrochemical detection of NPY within a nano-slit array}

The dielectrophoretic preconcentration of NPY $\left(300 \mathrm{~V}_{\mathrm{pp}} / \mathrm{cm}\right.$ at $3 \mathrm{MHz}$ with $\left.1.5 \mathrm{VDC} / \mathrm{cm}\right)$ occurs away from the high field points at the lateral constrictions within the nanochannel (Figure 24). As per the fluorescence images in Figure 24(a-f), it is apparent that NPY molecules are preconcentrated away from the constriction tip and onward over an extent of $\sim 100 \mu \mathrm{m}$ until the interface of the nanochannel to the adjoining microchannel. Within the assembled microfluidic device, the graphene-modified detection electrodes are aligned to fit on top of this DEP preconcentration region. Next, we

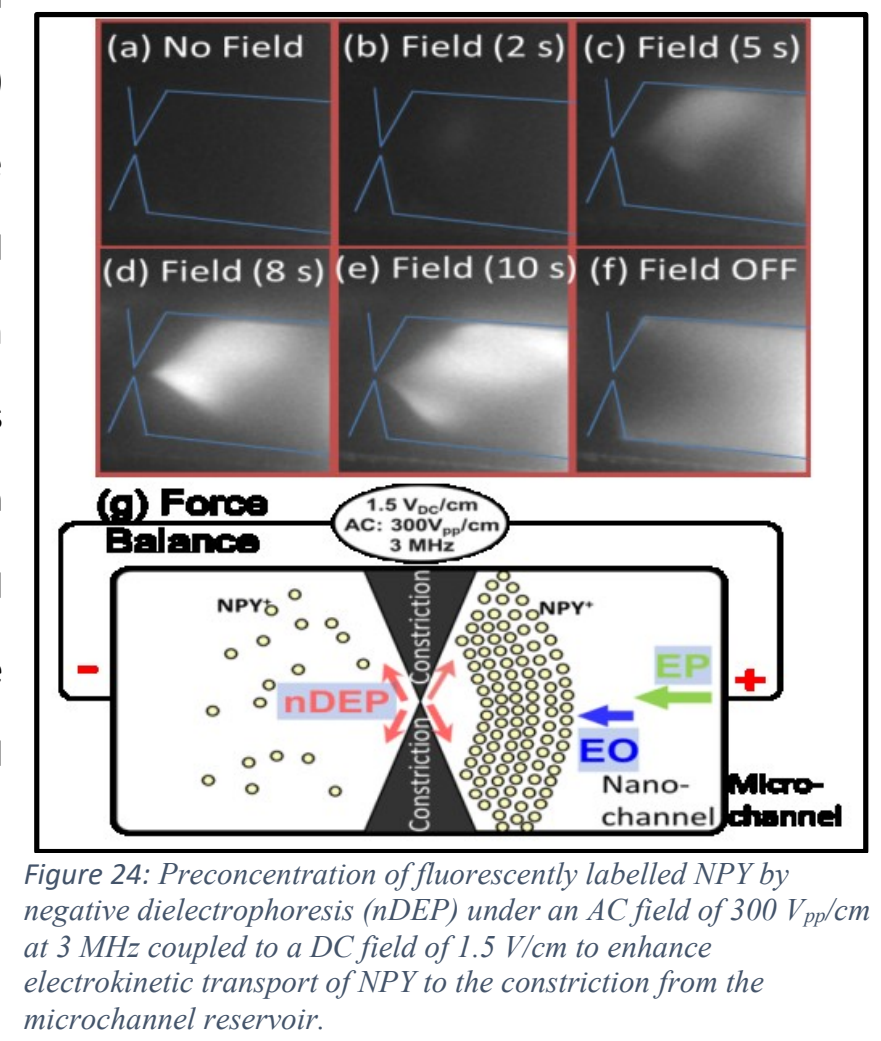


compare the detection accomplished by preconcentration of NPY through adsorptive accumulation under

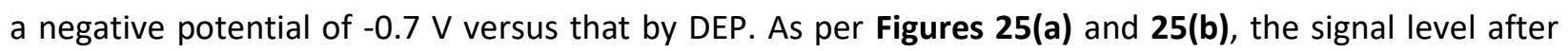
adsorptive accumulation for 300 seconds could be reached within 10 seconds of DEP preconcentration at both, $10 \mathrm{nM}$ and $10 \mathrm{pM}$ levels of NPY. Since ascorbic acid (AA, $\left.\mathrm{pK}_{\mathrm{a}}=4.17\right)$ and uric acid $\left(\mathrm{UA}, \mathrm{pK} \mathrm{a}_{\mathrm{a}}=5.7\right)$, exist in anionic form at pH 6.0 PBS, they are not adsorbed on NAF (a cation exchanger), especially within the 10 seconds required for dielectrophoretic preconcentration and detection of NPY at $32 \mathrm{pM}$, as apparent from the interference studies at $1 \mu \mathrm{M}$ ascorbic acid in Figure 25(c). Since dopamine and NPY are positively charged in the $\mathrm{pH} 6$ PBS buffer and since dopamine is much smaller than NPY, electrostatics under electrochemical accumulation causes dopamine peaks to dominate within interference studies of $100 \mathrm{nM}$ dopamine with 32 pM NPY. However, under dielectrophoresis, the cube-fold dependence of the trapping force on size ensures that only the larger NPY molecules are trapped, whereas the much smaller dopamine molecules can only get to the electrode by free diffusion. As per the interference studies in Figure 25(c) with $100 \mathrm{nM}$ dopamine and $32 \mathrm{pM}$ NPY, based on the comparable electrochemical signals for dopamine and NPY, we estimate
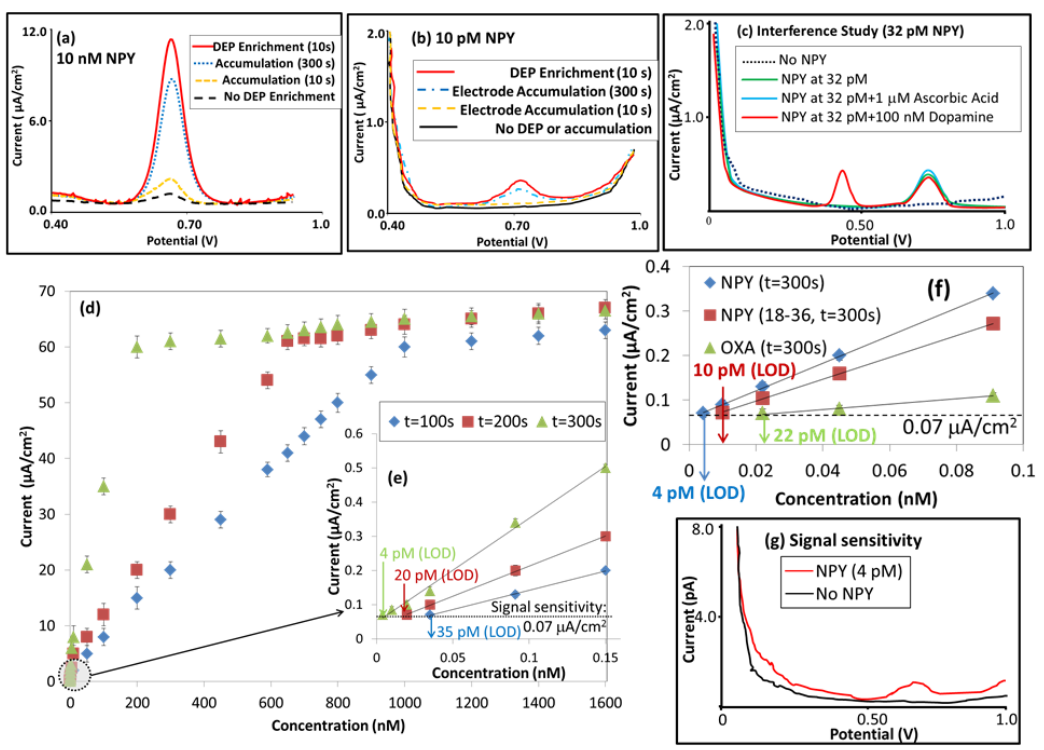

Figure 25: Signal enhancement after preconcentration by DEP versus electrochemical accumulation for various times: (a) $10 \mathrm{nM}$; and (b) $10 \mathrm{pMNPY}$. (c) Interference of $100 \mathrm{nM}$ Dopamine and $1 \mu \mathrm{M}$ Ascorbic Acid on the signal of 32 pM NPY; (d) \& (e): Enhancing limit of detection (LOD) for NPY to 4 pM (300 s accumulation); (f): LOD for NPY, NPY 18-36 and OXA (300 s accumulation); (g): Voltammogram at LOD for NPY (4 pM) after $10 \mathrm{~s}$ of DEP preconcentration on graphene-modified electrode of $10^{-5} \mathrm{~cm}^{2}$ area versus background (no NPY).

Figure 4(d) shows that while adsorptive accumulation for $100 \mathrm{~s}$ causes a wide linear region for signal versus analyte concentration, accumulation for $300 \mathrm{~s}$ causes a sharper rise of signal level with analyte concentration, thereby reaching the signal sensitivity level of $0.07 \mu \mathrm{A} / \mathrm{cm}^{2}$ at a lower concentration value [Figure 25(e)]. As a result, NPY can be detected down to 4 pM levels. To substantiate that the signal at the sensitivity level of $0.07 \mu \mathrm{A} / \mathrm{cm}^{2}$ ( 700 fA on $10^{-5} \mathrm{~cm}^{2}$ electrodes coupled to $\mathrm{nDEP}$ ) is greater than three 
times that of the background for characterizing limit of detection ${ }^{26}$, we show the voltammograms in Figure 25(g).

\section{Preconcentration and electrochemical detection of PSA within a nano-slit array}

The optimization of field conditions for nDEP enrichment of PSA, in conjunction to its effective binding to immobilized anti-PSA within the nanoslit device are confirmed using fluorescently labeled biomarkers. For this purpose, Dylight 488 labeled anti-PSA is immobilized over a patch on cover-slip glass that is aligned to the region with maximum biomarker enrichment under nDEP ( $\sim 5 \mathrm{~m}$ from constriction tips inside nanoslit). The fluorescence signal from intact immobilized anti-PSA capture probes and their binding with Dylight 594 labeled PSA $(20 \mu \mathrm{g} / \mathrm{mL})$ confirm that biofunctionality is preserved within the nanoslit after device bonding and assembly. After optimization of the applied field at $\sim 200 \mathrm{Vpp} / \mathrm{cm}$ at $6 \mathrm{MHz}$, we show the highly effective enrichment of Dylight 594 labeled PSA (starting level of $20 \mu \mathrm{g} / \mathrm{mL}$ ) in Figure 24a-24d. In fact, the fluorescence signal reaches a level equivalent to that from $500 \mu \mathrm{g} / \mathrm{mL}$ PSA (determined based on fluorescence of diluted stock), indicating a 25-fold PSA
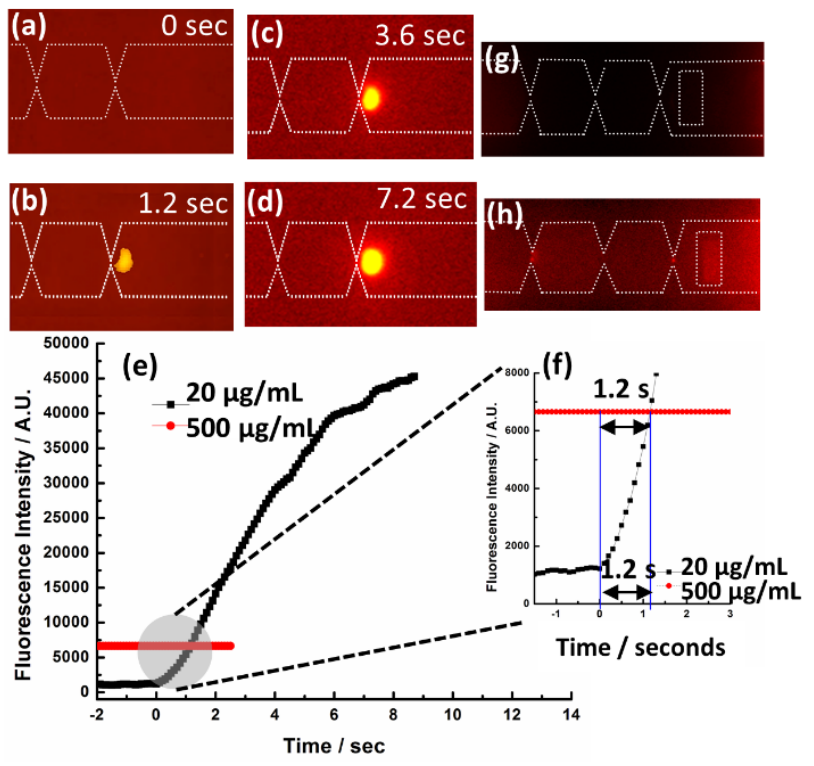

Figure 26: Enrichment of fluorescently labelled PSA biomarkers by negative dielectrophoresis ( $n D E P$ ) away from constrictions in the nanoslit: (a)-(d) images at indicated nDEP time intervals; (e) Average of maximum intensities from 20 pixels in nanoslit; ( $f$ ) shows $25 x$ enrichment in $1 \mathrm{~s}$ (black: $20 \mu \mathrm{g} / \mathrm{mL}$ PSA under $n D E P$; red: $500 \mu \mathrm{g} / \mathrm{mL}$ PSA with no enrichment). Binding of nDEP enriched PSA to immobilized anti-PSA capture probes in enrichment within just $1.2 \mathrm{~s}$ of $\mathrm{nDEP}$, with further nanoslit: ( $g$ ) before and ( $h$ ) after PSA enrichment (2 minutes).

steady enrichment over time (Figure 24e-24f). Fluorescence images in the molecular dam region with immobilized anti-PSA, prior to and following PSA enrichment after a PBS wash step (Figure $\mathbf{2 4 g}$ and $\mathbf{2 4 h}$, respectively), confirm a significant degree of enhancement in PSA binding. The measurement of PSA enrichment by fluorescence analysis is limited by non-specific binding (Figure $\mathbf{2 4 h}$ ) and the limited path length of light within the nanoslit, which limits sensitivity to the $\sim \mu \mathrm{g} / \mathrm{mL}$ range. 


\section{Influence of PSA enrichment on binding kinetics to anti-PSA}

In order to compare the binding kinetics of PSA with the immobilized anti-PSA capture probes on the working electrode surface three types of experiments were performed using electrochemical detection. Figure 26 summarizes voltammetry data from each set of experimental conditions: (i) $\sim 1 \mu \mathrm{L}$ scale droplet (dotted lines); (ii) the nanoslit device without nDEP enrichment (dashed lines); and (iii) the nanoslit device with nDEP enrichment (solid lines). The voltammetry platform shows a wide signal range,

from a sensitivity of $\sim 0.05 \mu \mathrm{A} / \mathrm{cm}^{2}$ to a signal saturation level of: $\sim 45 \mu \mathrm{A} / \mathrm{cm}^{2}$. The PSA concentration levels of $1 \mathrm{ng} / \mathrm{mL}$ (Figure 27a: blue curves) and $5 \mathrm{pg} / \mathrm{mL}$ (Figure 27b: green lines) were chosen, since these fall just at and below the $k_{d}$ value for PSA binding to anti-PSA [8], thereby making it possible to discern clearer differences in binding rate by addressing mass transport limitations. For case (i), the slow binding kinetics of PSA to anti-PSA in this concentration range is apparent from the rather slow rise in signal versus time. With $1 \mathrm{ng} / \mathrm{mL}$ of PSA (Figure 27a: blue dotted curve), the signal rises above the background level only after $\sim 200$ $s$ of binding time, reaching a steady-state level of $1.8 \mu \mathrm{A} / \mathrm{cm}^{2}$ after $1200 \mathrm{~s}$ (blue dotted horizontal line). With PSA at $5 \mathrm{pg} / \mathrm{mL}$ in the microliter droplet (Figure 27b: green dotted curve), the detection is ambiguous until $\sim 600 \mathrm{~s}$ of binding time, due to the large standard deviations at earlier times and due to the absence of a clear saturation in
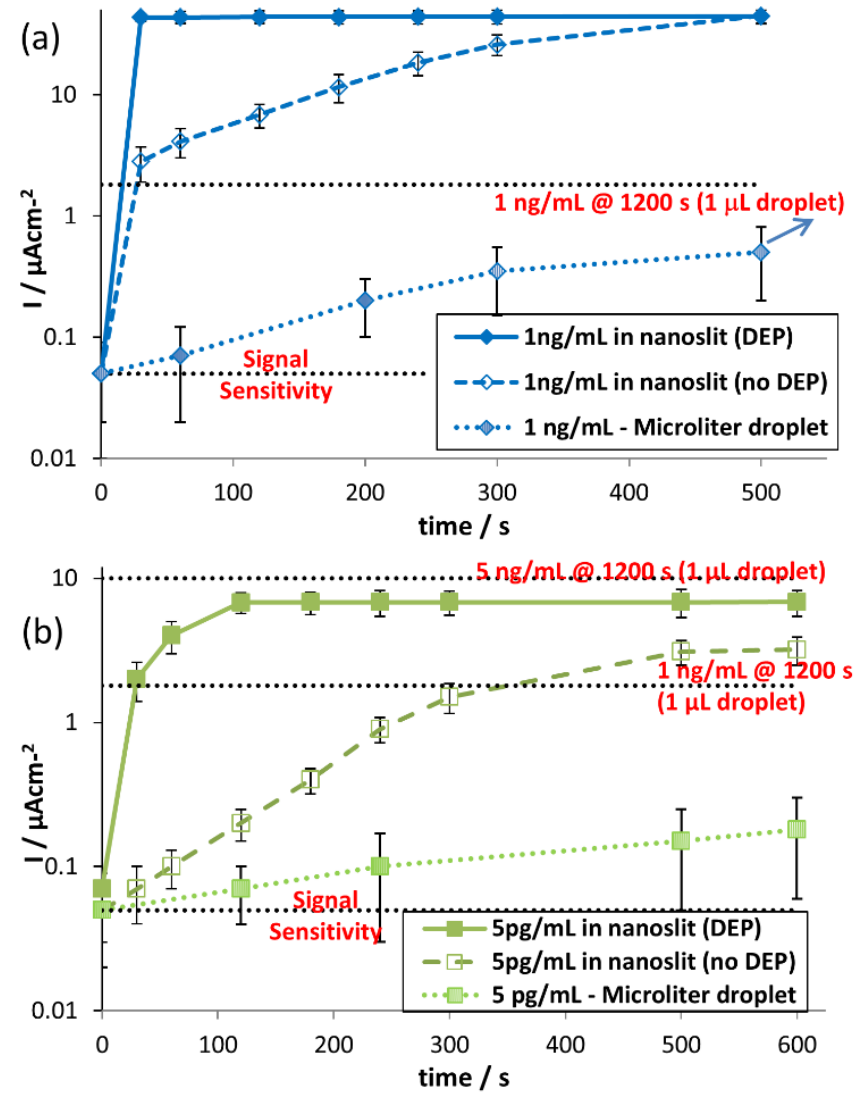

Figure 27: : Comparing PSA binding kinetics with immobilized antiPSA within: (i) microliter droplet (dotted line \& dotted symbols); (ii) nanoslit device in absence of nDEP enrichment (dashed lines \& open symbols); (iii) nanoslit device with nDEP enrichment (solid lines \& solid symbols) at PSA levels of: (a) $1 \mathrm{ng} / \mathrm{mL}$ : diamond symbols; and (b) $5 \mathrm{pg} / \mathrm{mL}$ : square symbols. Standard deviations are from three independent voltammetric measurements and the signal background determines the signal sensitivity at: 0.05 $\mu \mathrm{A} / \mathrm{cm}^{2}$. The signal levels achieved with $1 \mathrm{ng} / \mathrm{mL}$ and $5 \mathrm{ng} / \mathrm{mL} P S A$ at steady state (1200 s) using the microliter droplet incubation method (i) are shown as horizontal dotted lines for comparison with other methods (ii and iii). 
signal within the maximum measurement time of $1200 \mathrm{~s}$. The respective steady-state signal level after a binding time of $1200 \mathrm{~s}$ with $1 \mathrm{ng} / \mathrm{mL}$ and $5 \mathrm{ng} / \mathrm{mL}$ PSA, are shown for reference (Figure $\mathbf{2 7 b}$ : black dotted horizontal lines at 1.8 and $10 \mu \mathrm{A} / \mathrm{cm}^{2}$, respectively). For case (ii), the rapid enhancement of binding kinetics of PSA to anti-PSA immobilized inside the nanoslit is apparent from the sharp rise in signal within the first time point ( $\sim 30$ s of binding time) at PSA levels of $1 \mathrm{ng} / \mathrm{mL}$ (Figure 27a: dashed blue curve) and 5 $\mathrm{pg} / \mathrm{mL}$ (Figure $\mathbf{2 7 b}$ : dashed green curve), with signal saturation at $\sim 500 \mathrm{~s}$. Also, apparent is the reduction in standard deviation of the signals, especially as the signals approach a steady-state level of target binding to immobilized capture probes. For case (iii) under nDEP enrichment of PSA inside the nanoslit, the signal exhibits an even sharper rise, and reaches its steady-state level within $120 \mathrm{~s}$ with $5 \mathrm{pg} / \mathrm{mL}$ PSA (Figure 27b) and at well less than $30 \mathrm{~s}$ with $1 \mathrm{ng} / \mathrm{mL}$ PSA. It is also apparent that the standard deviations for the respective signals are minimal, especially as the signals approach their steady-state value.

Overall, enhancement of the binding kinetics causes signal saturation at a higher level and at significantly earlier times, with a higher level of enhancement for binding in the nanoslit versus the microliter droplet and further enhancement upon nDEP enrichment of PSA in the nanoslit. At detection limit levels, the onset of steady-state binding conditions is determined by the equilibrium between analyte association and dissociation [68], rather than by saturation of the immobilized capture probes. Hence, the steady-state signal at detection limit analyte levels can be increased by shifting the equilibrium in favor of analyte association [69], either by creating a concentrated analyte plug through nDEP enrichment of PSA and/or by enabling more effective analyte capture at the binding surface, as achieved within the nanoslit geometry under electrokinetic flow. By enabling nDEP enrichment in the nanoslit we can avail both these methods to further enhance the net signal saturation level. However, the net concentration level achieved under nDEP enrichment in the nanoslit drops with the starting PSA level, as apparent from the drop in steady-state signal level, even as the time to reach steady-state seems somewhat unchanged. Hence, while the steady-state signal level with $1 \mathrm{ng} / \mathrm{mL}$ PSA is enhanced under nDEP enrichment in the nanoslit from $\sim 1.8 \mu \mathrm{A} / \mathrm{cm}^{2}$ (within $\mu \mathrm{L}$ droplet) to $\geq 40 \mu \mathrm{A} / \mathrm{cm}^{2}$, which corresponds to signal from the near-complete binding of capture probes, the signal enhancement with $5 \mathrm{pg} / \mathrm{mL}$ PSA is more modest, with a steady-state 
signal level of $\sim 7 \mu \mathrm{A} / \mathrm{cm}^{2}$. Furthermore, the steady-state signal level using the nanoslit geometry with no nDEP enrichment is significantly lower $\left(\sim 3 \mu \mathrm{A} / \mathrm{cm}^{2}\right)$, suggesting a sharper influence of enhancement of concentration versus enhancement of analyte capture on shifting the equilibrium in favor of analyte association. The inability of nDEP enrichment to cause steady-state signals at the capture probe signal saturation level, even upon prolonged nDEP enrichment can be attributed to limitations from backdiffusion, electrothermal flow under Joule heating, and possibly from conformation incompatibility of some fraction of the enriched PSA molecules to immobilized anti-PSA. Hence, improvement of the detection sensitivity under nDEP enrichment is determined by whether this enhanced steady-state level falls above the signal sensitivity level. The degree of nDEP enrichment based on the steady-state signal is quantified in Figure 27b. A starting level of $5 \mathrm{pg} / \mathrm{mL}$ of PSA (green solid line or case (iii)) saturates within $120 \mathrm{~s}$ at a signal level similar to that achieved with free diffusion (case (i)) using $5 \mathrm{ng} / \mathrm{mL}$ PSA (black dotted line). This suggests that a dilute plug with $5 \mathrm{pg} / \mathrm{mL}$ PSA exhibits signal characteristics somewhat similar to a concentrated plug with $\sim 1000 x$ PSA enrichment in the vicinity of anti-PSA capture probes, due to nDEP trapping in the nanoslit device over $120 \mathrm{~s}$.

\section{Enhancing sensitivity for PSA detection}

While enhancement of PSA binding kinetics under nDEP enrichment leads to earlier signal saturation, the net steady-state signal falls with lower starting PSA levels. Hence, improvements to the limit of detection (LOD) based on whether the enhanced steady-state signal at lower PSA levels falls above signal sensitivity were explored. From signal levels in Figure 27b, it is apparent that PSA detection at the $5 \mathrm{pg} / \mathrm{mL}$ level in the $\mu \mathrm{L}$ droplet is ambiguous, whereas it is clear within the nanoslit device and is further

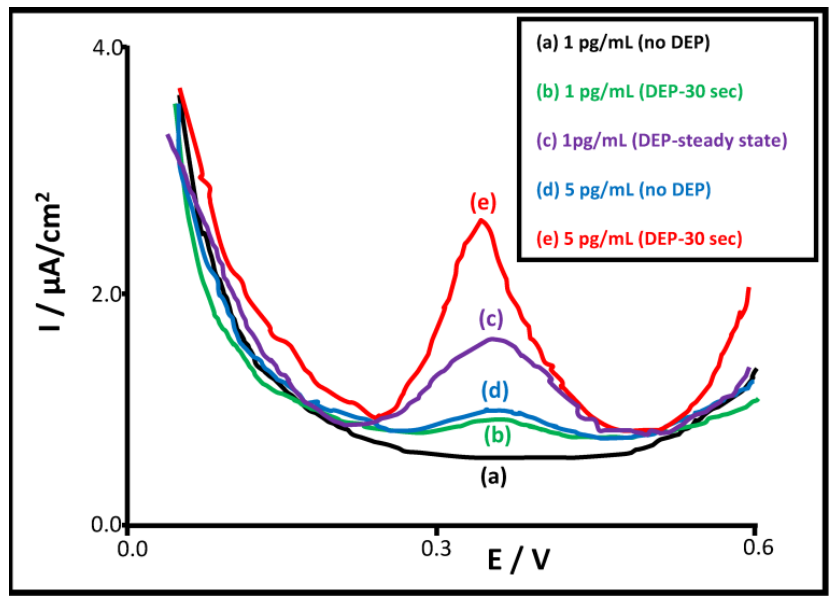

Figure 28: Square wave voltammetry scans for PSA binding with anti-PSA immobilized in nanoslit device at PSA levels of $5 \mathrm{pg} / \mathrm{mL}$ (without and with $n D E P$ enrichment over $30 \mathrm{~s}$ binding time in: $d$ \& e) and $1 \mathrm{pg} / \mathrm{mL}$ (without nDEP enrichment (a) and with nDEP enrichment for $30 \mathrm{~s}$ (b) and for $120 \mathrm{~s}(\mathrm{c})$ ). 
sped-up upon nDEP enrichment. This difference is also apparent in the voltammetric scans after $30 \mathrm{~s}$ of binding time with $5 \mathrm{pg} / \mathrm{mL}$ of PSA within the nanoslit device, in absence (Figure 28d) and with nDEP enrichment (Figure 28e). The signal levels (after $30 \mathrm{~s}$ binding time) are higher with nDEP enrichment and the standard deviations are lower (see Figure $\mathbf{2 7 b}$ : solid square symbol at $2 \mu \mathrm{A} / \mathrm{cm}^{2}$ versus open square symbol at $0.08 \mu \mathrm{A} / \mathrm{cm}^{2}$ that barely rises above the signal sensitivity). On the other hand, PSA levels of 1 $\mathrm{pg} / \mathrm{mL}$ cannot be detected on the nanoslit device in the absence of nDEP enrichment (Figure 28a). After $30 \mathrm{~s}$ of nDEP enrichment in the nanoslit device, the signal barely rises beyond the sensitivity level (Figure 28b), while after 2 minutes of nDEP enrichment, a steady-state signal well above the background is apparent (Figure 28c). Hence, the earlier signal saturation caused by nDEP enrichment of PSA in the nanoslit, improves sensitivity to enable a $1 \mathrm{pg} / \mathrm{mL}$ detection limit in PBS media. 


\section{Chapter 4: Conclusion and Future Work}

\section{Conclusion}

Within this dissertation, we demonstrated the design, development, and operation of novel micro and nanofluidic device platforms for two clinically significant applications. In first application, we demonstrate a method to achieve the deformability-based separation of pancreatic islets of Langerhans from contaminating acinar tissue. Despite its potential as a powerful treatment for T1 Diabetes the transplantation of pancreatic islet cell aggregates is limited by a heightened immune response and poor tissue engraftment. We demonstrated that the current density gradient based separation method is shown to result in a transplant plug with $60 \%$ or less of islet purity, due to the wide distribution of islets across the centrifuged sample bins, which likely occur due to the density overlap between islets and a significant portion of acinar particles. Hence, due to the relatively sparse islet levels in a given bin, there is a need to combine samples from multiple bins, thereby causing significant acinar tissue levels within the final transplant plug that exacerbates immune rejection. We further demonstrate that there is a minimal overlap in the deformability levels of islet versus acinar tissue populations harvested from mice. This enabled the selective isolation of islets versus acinar tissue, based on the bypass pressure required to deform the respective aggregate through bifurcating junctions within a microfluidic device. In order to produce a continuous separation method based on this, we designed a two-cycle microfluidic device wherein the hydrodynamic flow resistance towards the outlet is switched from a "high" level to enable a separation mode for removal of deformable acinar populations, to a "low" level to enable a collection mode for isolation of rigid islets trapped at bifurcating points. This separation device is shown to be capable of enriching islets from relatively dilute starting levels up to purity levels that meet transplant criteria. Furthermore, we demonstrate that the device is capable of enhancing islet purity of starting samples with similar islet purities as those obtained from the standard density gradient based separation. significantly reduced acinar levels with little loss of islet tissue. Purified islets isolated by the microfluidic

method are shown to be viable and capable of transplantation, as measured by angiogenesis within a murine retinal implant model. We further substantiated our initial results with mouse tissue by repeating 
the threshold bypass pressure measurements with tissue derived from human donor organs. We found that human tissue exhibits a higher size and deformability range than tissue harvested from mice. While the deformability overlap between the human acinar and islet tissue is more pronounced than with the mouse tissue, the overlap is still shown to be minimal. We envision the application of this microfluidic strategy towards generating small-volume transplant plugs, with high islet purity and significantly reduced acinar levels for alleviating immune responses after transplantation leading to improved transplant outcomes.

In the second application, we demonstrated the integration of frequency selective electrokinetic preconcentration with electrochemical detection within a nanoslit platform for ultrafast low sample volume measurement of the proteomic biomarkers neuropeptide $Y$ (NPY) and prostate specific antigen PSA. This platform overcomes limitations caused by poor mass transport of analyte through nanoslit confinement, which eliminates diffusion boundary layers, and by creating a highly concentrated plug of the biomarker at the working electrode surface, through enrichment under a molecular dam scheme with negative dielectrophoresis (nDEP). Utilizing this platform, we demonstrated the ability to rapidly preconcentrate both proteomic biomarkers utilizing a dielectrophoretic molecular damming method, traping analyte away from the sharp lateral insulator constrictions and onto graphene-modified electrodes that had been aligned within the nanochannel. For NPY this was achieved using a $3 \mathrm{MHz} A C$ field of $300 \mathrm{~V}_{\mathrm{pp}} / \mathrm{cm}$ alongside a $1.5 \mathrm{VDC} / \mathrm{cm}$. Utilizing electrodes that had been optimized for fast adsorption kinetics preconcentrated NPY was detected through electrochemical oxidation of their tyrosine moiety from sub-nanoliter initial sample volumes at sensitivity levels down to $4 \mathrm{pM}$. For PSA preconcentration was achieved using a $6 \mathrm{MHz} A C$ field of $200 \mathrm{Vpp} / \mathrm{cm}$ alongside a $1.5 \mathrm{~V} / \mathrm{cm}$ DC field for PSA. Anti-PSA capture probes were bound to the graphene modified working electrode surface within the nanoslit platform, in order to facilitate electrochemical detection through a sandwich assay. The nDEP enriched PSA biomarkers were shown to effectively bind to anti-PSA capture probes immobilized on the nanoslit device. By comparing the binding kinetics of PSA ( $5 \mathrm{pg} / \mathrm{mL}-1 \mathrm{ng} / \mathrm{mL}$ range) to anti-PSA in a microdroplet versus 
on a nanoslit device, with and without nDEP enrichment, we demonstrated the steep enhancement in signal versus time on the nanoslit platform, with steady-state signal levels achieved within just 2 minutes upon nDEP enrichment.

\section{Aim One Future Work: Deformability based separation of islets}

\section{Adaptation of separation device to human Islet and acinar samples}

Within this dissertation, we demonstrated that there exists a significant difference in the deformabilities of populations of mouse derived pancreatic islets versus those of acinar tissue. Utilizing this, we developed a microfluidic method for the deformability based separation of mouse derived islets from acinar tissue and demonstrated the ability to achieve improved islet purity for samples with two application relevant starting purities. We further validated this approach for applications in transplant therapies by demonstrating a similar difference between the deformability of islets versus acinar tissue from human donor tissue. However, while the separation methodology may be applied to human derived tissue the current separation chip must be adapted to accommodate the larger size and higher pressures required for human tissue separation. Furthermore, due to the increase in particle size with human derived tissue, heightened acinar to islet tissue

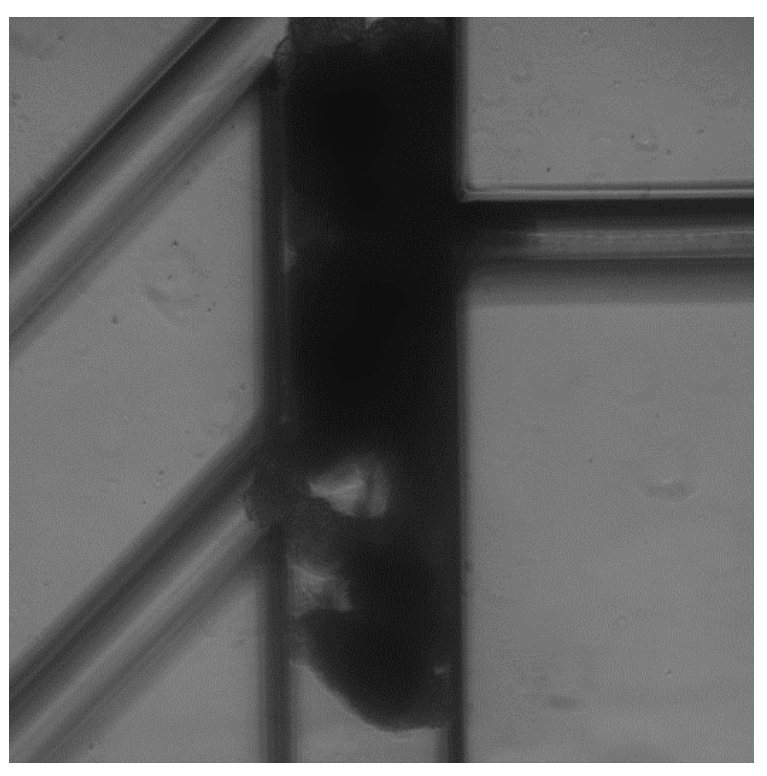

Figure 29: Chip Clogging due to increased size range of human islets and islet to acinar aggregation aggregation and clogging have been isolated has been observed leading to device clogging and overall poor separation (figure 29). Therefore, we propose a scaled-up version of the current separation device to account for the larger particle size and higher flowrates associated with human tissue separation. 
Furthermore we propose the integration of a presorting anti-aggregation stage utilizing Dimond shaped posts to prevent particle to particle interactions leading to device clogging.

\section{Aim Two Future Work: Electrokinetic preconcentration and electrochemical detection within a nanoslit platform}

Within this dissertation, we demonstrated the integration of electrokinetic preconsentration with electrochemical sensing methods within a fused silica nano-slit. In this section we propose to further advance the versatility of this method through the replacement of the micro/nano fabricated fused silica nano-fluidic platform with a thermoplastic replica fabricated via Nano-imprint lithography.

\section{Drawbacks of current fabrication methods and materials}

While fused silica chips fabricated via the method described above have been successfully realized, several significant drawbacks with this manufacturing modality exist. Micro/nanochannel formation using the processes described above is an extremely high cost and time consuming procedure. Not only does is necessitate the use of several high cost materials such as fused silica wafers, multiple photoresists, and expensive process gasses, but it also requires extremely specialized equipment and training not readily available. This high cost is further compounded by the multiple defect sources associated with high precision pattern alignment

\section{Damage due to joule} heating

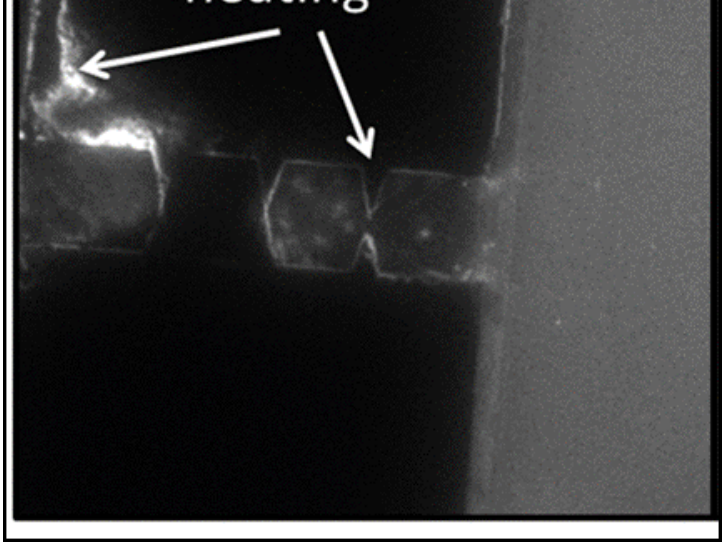

Figure 30: damage to PSQ adhesion layer due to joule heating during preconcentration step.

steps and destructive processes such as sand blasting, which significantly lower yield and lead to inconsistent device quality and structure. Finally the chips suffer from an extremely low shelf life as the 
effects of $\mathrm{O} 2$ plasma treatment also degrade rapidly making device operation impossible 24 hours after assembly.

Another major bottleneck is due to the extremely low yield and fragile nature of the PSQ bonding process. Physical bonding of two rigid materials is highly dependent on contamination. This is especially true in cases requiring bonding between surfaces with low contact surface area such as the nanoconstrictions in the fused silica chip. Because of this, yield from the bonding process is extremely low. Even in successful bonding cases variation in quality is extreme and may result in inconsistencies in experimental data and greater susceptibility to damage from joule heating. In general, the PSQ bond is extremely fragile and degrades under electric field due to joule heating, especially under low frequency or DC fields needed to drive material into the Nano-slit (figure $\mathbf{3 0}$ ).

\section{Nano-Imprint Lithography}

Nano-Imprint Lithography, or NIL, is a method to construct micro/nanopatterned replicas from a master or stamp through the mechanical deformation of an imprintable material. One common NIL methodology is Hot Embossing (HE NIL) which replicates patterns in a thermoplastic. Thermoplastics are polymers that become pliable above a certain temperature, known as the Glass Transition Temperature or $\left(T_{g}\right)$. The HE NIL process utilizes pressure to replicate the negative of a stamp in thermoplastic heated above its Tg. Once imprinted, the Thermoplastic is allowed to cool and the Stamp is de-embossed, thereby leaving a negative 3D pattern imprinted in the thermoplastic. In order to extend the lifetime of the imprint stamp, a soft stamp manufactured from an elastomeric polymer layer adhered to a borosilicate back plain is typically used. This promotes easier non-destructive embossing, and allows for fast stamp fabrication using a micro-molding replication process. Rigid hard stamps may be used; however this often results in damage to the imprinted mold or the stamp and is therefore not recommended. As imprint fidelity is largely dependent on the uniformity of pressure applied to the stamp, a high precision piston is used to apply force to the thermoplastic/stamp stack and a graphite compliance layer is also often included in between the stamp backing and the piston. Furthermore imprinting is done under vacuum and within a 
clean room environment. This method of replication is ideal for fabrication of the Nano-slit molecular dam device due to its low cost and high throughput as well as its ability to replicate nano-scale features with high resolution.[70] 


\section{Work Cited}

[1] B. J. Sanghavi, W. Varhue, A. Rohani, K. T. Liao, L. A. L. Bazydlo, C. F. Chou, et al., "Ultrafast immunoassays by coupling dielectrophoretic biomarker enrichment in nanoslit channel with electrochemical detection on graphene (vol 15, pg 4563, 2015), "Lab on a Chip, vol. 15, pp. 46264626, 2015.

[2] B. J. Sanghavi, W. Varhue, J. L. Chavez, C. F. Chou, and N. S. Swami, "Electrokinetic Preconcentration and Detection of Neuropeptides at Patterned Graphene-Modified Electrodes in a Nanochannel," Analytical Chemistry, vol. 86, pp. 4120-4125, May 62014.

[3] K. T. Liao and C. F. Chou, "Nanoscale Molecular Traps and Dams for Ultrafast Protein Enrichment in High-Conductivity Buffers," Journal of the American Chemical Society, vol. 134, pp. 8742-8745, May 302012.

[4] K. T. Liao, M. Tsegaye, V. Chaurey, C. F. Chou, and N. S. Swami, "Nano-constriction device for rapid protein preconcentration in physiological media through a balance of electrokinetic forces," Electrophoresis, vol. 33, pp. 1958-1966, Jul 2012.

[5] Y. H. Su, M. Tsegaye, W. Varhue, K. T. Liao, L. S. Abebe, J. A. Smith, et al., "Quantitative dielectrophoretic tracking for characterization and separation of persistent subpopulations of Cryptosporidium parvum, "Analyst, vol. 139, pp. 66-73, 2014.

[6] Y. H. Su, A. Rohani, C. A. Warren, and N. S. Swami, "Tracking Inhibitory Alterations during Interstrain Clostridium difficile Interactions by Monitoring Cell Envelope Capacitance," Acs Infectious Diseases, vol. 2, pp. 544-551, Aug 2016.

[7] Y. H. Su, C. A. Warren, R. L. Guerrant, and N. S. Swami, "Dielectrophoretic Monitoring and Interstrain Separation of Intact Clostridium difficile Based on Their S(Surface)-Layers," Analytical Chemistry, vol. 86, pp. 10855-10863, Nov 42014.

[8] M. S. Rogers, A. E. Birsner, and R. J. D'Amato, "The mouse cornea micropocket angiogenesis assay," Nat Protoc, vol. 2, pp. 2545-50, 2007.

[9] M. S. Rogers, A. E. Birsner, and R. J. D'amato, "The mouse cornea micropocket angiogenesis assay," Nature protocols, vol. 2, pp. 2545-2550, 2007.

[10] M. Paschen, T. Moede, B. Leibiger, S. Jacob, G. Bryzgalova, I. B. Leibiger, et al., "Non-invasive cell type selective in vivo monitoring of insulin resistance dynamics, "Scientific reports, vol. 6, 2016.

[11] P. R. Nair and M. A. Alam, "Performance limits of nanobiosensors," Applied Physics Letters, vol. 88, Jun 52006.

[12] P. E. Sheehan and L. J. Whitman, "Detection limits for nanoscale biosensors," Nano Letters, vol. 5, pp. 803-807, Apr 2005.

[13] B. J. Sanghavi, O. S. Wolfbeis, T. Hirsch, and N. S. Swami, "Nanomaterial-based electrochemical sensing of neurological drugs and neurotransmitters," Microchimica Acta, vol. 182, pp. 1-41, Jan 2015.

[14] W. Sparreboom, A. van den Berg, and J. C. T. Eijkel, "Principles and applications of nanofluidic transport," Nature Nanotechnology, vol. 4, pp. 713-720, Nov 2009.

[15] V. Chaurey, A. Rohani, Y. H. Su, K. T. Liao, C. F. Chou, and N. S. Swami, "Scaling down constrictionbased (electrodeless) dielectrophoresis devices for trapping nanoscale bioparticles in physiological media of high-conductivity," Electrophoresis, vol. 34, pp. 1097-1104, Apr 2013.

[16] P. Chhabra and K. L. Brayman, "Overcoming barriers in clinical islet transplantation: current limitations and future prospects," Curr Probl Surg, vol. 51, pp. 49-86, Feb 2014.

[17] A. American Diabetes, "Economic costs of diabetes in the U.S. in 2012," Diabetes Care, vol. 36, pp. 1033-46, Apr 2013. 
[18] T. M. Dall, S. E. Mann, Y. Zhang, W. W. Quick, R. F. Seifert, J. Martin, et al., "Distinguishing the economic costs associated with type 1 and type 2 diabetes, "Popul Health Manag, vol. 12, pp. 10310, Apr 2009.

[19] P. R. Johnson and K. E. Jones, "Pancreatic islet transplantation," Semin Pediatr Surg, vol. 21, pp. 272-80, Aug 2012.

[20] F. B. Barton, M. R. Rickels, R. Alejandro, B. J. Hering, S. Wease, B. Naziruddin, et al., "Improvement in outcomes of clinical islet transplantation: 1999-2010," Diabetes Care, vol. 35, pp. 1436-45, Jul 2012.

[21] R. M. Langer, "Islet transplantation: lessons learned since the Edmonton breakthrough," Transplant Proc, vol. 42, pp. 1421-4, Jun 2010.

[22] C. Ricordi and T. B. Strom, "Clinical islet transplantation: advances and immunological challenges," Nat Rev Immunol, vol. 4, pp. 259-68, Apr 2004.

[23] R. M. Langer, "Islet Transplantation: Lessons Learned Since the Edmonton Breakthrough," Transplantation Proceedings, vol. 42, pp. 1421-1424, Jun 2010.

[24] M. Yamada, M. Nakashima, and M. Seki, "Pinched flow fractionation: Continuous size separation of particles utilizing a laminar flow profile in a pinched microchannel," Analytical Chemistry, vol. 76, pp. 5465-5471, Sep 152004.

[25] M. L. Jiang, K. Budzan, and G. Drazer, "Fractionation by shape in deterministic lateral displacement microfluidic devices," Microfluidics and Nanofluidics, vol. 19, pp. 427-434, Aug 2015.

[26] M. E. Myrand-Lapierre, X. Y. Deng, R. R. Ang, K. Matthews, A. T. Santoso, and H. S. Ma, "Multiplexed fluidic plunger mechanism for the measurement of red blood cell deformability, "Lab on a Chip, vol. 15, pp. 159-167, 2015.

[27] J. M. Jackson, J. B. Taylor, M. A. Witek, S. A. Hunsucker, J. P. Waugh, Y. Fedoriw, et al., "Microfluidics for the detection of minimal residual disease in acute myeloid leukemia patients using circulating leukemic cells selected from blood, "Analyst, vol. 141, pp. 640-651, 2016.

[28] S. E. Weigum, P. N. Floriano, N. Christodoulides, and J. T. McDevitt, "Cell-based sensor for analysis of EGFR biomarker expression in oral cancer," Lab on a Chip, vol. 7, pp. 995-1003, 2007.

[29] H. W. Wu, C. C. Lin, and G. B. Lee, "Stem cells in microfluidics," Biomicrofluidics, vol. 5, Mar 2011.

[30] D. R. Gossett, W. M. Weaver, A. J. Mach, S. C. Hur, H. T. K. Tse, W. Lee, et al., "Label-free cell separation and sorting in microfluidic systems, "Analytical and Bioanalytical Chemistry, vol. 397, pp. 3249-3267, Aug 2010.

[31] C. W. Shields, C. D. Reyes, and G. P. Lopez, "Microfluidic cell sorting: a review of the advances in the separation of cells from debulking to rare cell isolation, "Lab on a Chip, vol. 15, pp. 1230-1249, 2015.

[32] J. Berthier and P. Silberzan, Microfluidics for biotechnology, 2nd ed. Boston: Artech House, 2010.

[33] D. W. Inglis, "Efficient microfluidic particle separation arrays," Applied Physics Letters, vol. 94, Jan 52009.

[34] H. Mohamed, J. N. Turner, and M. Caggana, "Biochip for separating fetal cells from maternal circulation," Journal of Chromatography A, vol. 1162, pp. 187-192, Aug 312007.

[35] S. M. McFaul, B. K. Lin, and H. Ma, "Cell separation based on size and deformability using microfluidic funnel ratchets," Lab Chip, vol. 12, pp. 2369-76, Jul 72012.

[36] J. Seo, M. H. Lean, and A. Kole, "Membrane-free microfiltration by asymmetric inertial migration," Applied Physics Letters, vol. 91, Jul 162007.

[37] M. Yamada, K. Kano, Y. Tsuda, J. Kobayashi, M. Yamato, M. Seki, et al., "Microfluidic devices for size-dependent separation of liver cells, " Biomedical Microdevices, vol. 9, pp. 637-645, Oct 2007.

[38] S. Yang, A. Undar, and J. D. Zahn, "A microfluidic device for continuous, real time blood plasma separation," Lab on a Chip, vol. 6, pp. 871-880, 2006. 
[39] B. Roda, A. Zattoni, P. Reschiglian, M. H. Moon, M. Mirasoli, E. Michelini, et al., "Field-flow fractionation in bioanalysis: A review of recent trends, "Analytica Chimica Acta, vol. 635, pp. 132143, Mar 92009.

[40] K. W. Oh, K. Lee, B. Ahn, and E. P. Furlani, "Design of pressure-driven microfluidic networks using electric circuit analogy," Lab on a Chip, vol. 12, pp. 515-545, 2012.

[41] M. Fornal, M. Lekka, G. Pyka-Fosciak, K. Lebed, T. Grodzicki, B. Wizner, et al., "Erythrocyte stiffness in diabetes mellitus studied with atomic force microscope," Clinical Hemorheology and Microcirculation, vol. 35, pp. 273-276, 2006.

[42] J. Guck, R. Ananthakrishnan, H. Mahmood, T. J. Moon, C. C. Cunningham, and J. Kas, "The optical stretcher: A novel laser tool to micromanipulate cells, "Biophysical Journal, vol. 81, pp. 767-784, Aug 2001.

[43] R. M. Hochmuth, "Micropipette aspiration of living cells, "Journal of Biomechanics, vol. 33, pp. 1522, Jan 2000.

[44] Y. Zheng, J. Nguyen, Y. Wei, and Y. Sun, "Recent advances in microfluidic techniques for single-cell biophysical characterization," Lab on a Chip, vol. 13, pp. 2464-2483, 2013.

[45] P. R. Baraniak, M. T. Cooke, R. Saeed, M. A. Kinney, K. M. Fridley, and T. C. McDevitt, "Stiffening of human mesenchymal stem cell spheroid microenvironments induced by incorporation of gelatin microparticles," Journal of the Mechanical Behavior of Biomedical Materials, vol. 11, pp. 63-71, Jul 2012.

[46] D. Qi, D. J. Hoelzle, and A. C. Rowat, "Probing single cells using flow in microfluidic devices," European Physical Journal-Special Topics, vol. 204, pp. 85-101, Apr 2012.

[47] Y. Zheng, J. Nguyen, C. Wang, and Y. Sun, "Electrical measurement of red blood cell deformability on a microfluidic device, "Lab on a Chip, vol. 13, pp. 3275-3283, 2013.

[48] O. Otto, P. Rosendahl, A. Mietke, S. Golfier, C. Herold, D. Klaue, et al., "Real-time deformability cytometry: on-the-fly cell mechanical phenotyping," Nature Methods, vol. 12, pp. 199-+, Mar 2015.

[49] J. S. Kuo, Y. X. Zhao, P. G. Schiro, L. Y. Ng, D. S. W. Lim, J. P. Shelby, et al., "Deformability considerations in filtration of biological cells," Lab on a Chip, vol. 10, pp. 837-842, 2010.

[50] S. M. McFaul, B. K. Lin, and H. S. Ma, "Cell separation based on size and deformability using microfluidic funnel ratchets, "Lab on a Chip, vol. 12, pp. 2369-2376, 2012.

[51] X. Chen, D. F. Cui, C. C. Liu, H. Li, and Z. X. Geng, "Microfluidic cell separation chips based on crossflow filtration," Chemical Journal of Chinese Universities-Chinese, vol. 28, pp. 59-61, Jan 10 2007.

[52] A. Rohani, W. Varhue, Y. H. Su, and N. S. Swami, "Electrical tweezer for highly parallelized electrorotation measurements over a wide frequency bandwidth," Electrophoresis, vol. 35, pp. 1795-1802, Jul 2014.

[53] C. F. Chou, J. O. Tegenfeldt, O. Bakajin, S. S. Chan, E. C. Cox, N. Darnton, et al., "Electrodeless dielectrophoresis of single- and double-stranded DNA," Biophysical Journal, vol. 83, pp. 21702179, Oct 2002.

[54] N. Swami, C. F. Chou, V. Ramamurthy, and V. Chaurey, "Enhancing DNA hybridization kinetics through constriction-based dielectrophoresis," Lab on a Chip, vol. 9, pp. 3212-3220, 2009.

[55] V. Chaurey, C. Polanco, C. F. Chou, and N. S. Swami, "Floating-electrode enhanced constriction dielectrophoresis for biomolecular trapping in physiological media of high conductivity," Biomicrofluidics, vol. 6, Mar 2012.

[56] V. Farmehini, A. Rohani, Y. H. Su, and N. S. Swami, "A wide-bandwidth power amplifier for frequency-selective insulator-based dielectrophoresis," Lab on a Chip, vol. 14, pp. 4183-4187, 2014. 
[57] A. Rohani, W. Varhue, Y. H. Su, and N. S. Swami, "Quantifying spatio-temporal dynamics of biomarker pre-concentration and depletion in microfluidic systems by intensity threshold analysis," Biomicrofluidics, vol. 8, Sep 2014.

[58] A. Rohani, W. Varhue, K. T. Liao, C. F. Chou, and N. S. Swami, "Nanoslit design for ion conductivity gradient enhanced dielectrophoresis for ultrafast biomarker enrichment in physiological media," Biomicrofluidics, vol. 10, May 2016.

[59] N. E. Hebert, B. Snyder, R. L. McCreery, W. G. Kuhr, and S. A. Brazill, "Performance of pyrolyzed photoresist carbon films in a microchip capillary electrophoresis device with sinusoidal voltammetric detection, "Analytical Chemistry, vol. 75, pp. 4265-4271, Aug 152003.

[60] S. J. Li, D. H. Deng, Q. Shi, and S. R. Liu, "Electrochemical synthesis of a graphene sheet and gold nanoparticle-based nanocomposite, and its application to amperometric sensing of dopamine," Microchimica Acta, vol. 177, pp. 325-331, Jun 2012.

[61] B. J. Polk, A. Stelzenmuller, G. Mijares, W. MacCrehan, and M. Gaitan, "Ag/AgCl microelectrodes with improved stability for microfluidics," Sensors and Actuators B-Chemical, vol. 114, pp. 239247, Mar 302006.

[62] M. P. Chatrathi, J. Wang, and G. E. Collins, "Sandwich electrochemical immunoassay for the detection of Staphylococcal enterotoxin B based on immobilized thiolated antibodies, "Biosensors \& Bioelectronics, vol. 22, pp. 2932-2938, Jun 152007.

[63] T. Leichle and C. F. Chou, "Biofunctionalized nanoslits for wash-free and spatially resolved realtime sensing with full target capture," Biomicrofluidics, vol. 9, May 2015.

[64] D. S. Moore, "Amino-Acid and Peptide Net Charges - a Simple Calculational Procedure," Biochemical Education, vol. 13, pp. 10-11, 1985.

[65] R. P. Liu, C. Wang, Q. Jiang, W. Zhang, Z. Yue, and G. H. Liu, "Magnetic-particle-based, ultrasensitive chemiluminescence enzyme immunoassay for free prostate-specific antigen," Analytica Chimica Acta, vol. 801, pp. 91-96, Nov 12013.

[66] H. Ichii, A. Pileggi, R. D. Molano, D. A. Baidal, A. Khan, Y. Kuroda, et al., "Rescue purification maximizes the use of human islet preparations for transplantation," American Journal of Transplantation, vol. 5, pp. 21-30, Jan 2005.

[67] D. W. R. Gray, R. Sutton, P. Mcshane, M. Peters, and P. J. Morris, "Exocrine Contamination Impairs Implantation of Pancreatic-Islets Transplanted beneath the Kidney Capsule," Journal of Surgical Research, vol. 45, pp. 432-442, Nov 1988.

[68] D. Leboeuf and N. Henry, "Molecular bond formation between surfaces: Anchoring and shearing effects," Langmuir, vol. 22, pp. 127-133, Jan 32006.

[69] M. Zimmermann, E. Delamarche, M. Wolf, and P. Hunziker, "Modeling and optimization of highsensitivity, low-volume microfluidic-based surface immunoassays," Biomedical Microdevices, vol. 7, pp. 99-110, Jun 2005.

[70] R. Chantiwas, M. L. Hupert, S. R. Pullagurla, S. Balamurugan, J. Tamarit-Lopez, S. Park, et al., "Simple replication methods for producing nanoslits in thermoplastics and the transport dynamics of double-stranded DNA through these slits," Lab on a Chip, vol. 10, pp. 3255-3264, 2010. 